\title{
Conjectures and results about parabolic induction of representations of $\mathrm{GL}_{n}(F)$
}

\author{
Erez Lapid ${ }^{1}$ (1) * Alberto Mínguez ${ }^{2}$ (1)
}

Received: 22 December 2019 / Accepted: 4 June 2020 / Published online: 6 July 2020

(C) The Author(s) 2020

\begin{abstract}
In 1980 Zelevinsky introduced certain commuting varieties whose irreducible components classify complex, irreducible representations of the general linear group over a non-archimedean local field with a given supercuspidal support. We formulate geometric conditions for certain triples of such components and conjecture that these conditions are related to irreducibility of parabolic induction. The conditions are in the spirit of the Geiss-Leclerc-Schröer condition that occurs in the conjectural characterization of $\square$-irreducible representations. We verify some special cases of the new conjecture and check that the geometric and representation-theoretic conditions are compatible in various ways.
\end{abstract}

\section{Contents}

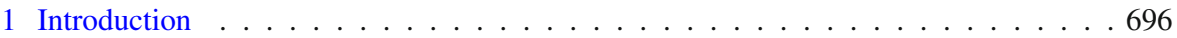

2 Notation and preliminaries . . . . . . . . . . . . . . . . . . . 699

3 The conditions $\operatorname{SA}\left(\mathfrak{m}, \mathfrak{m}^{\prime}\right)$ and $\operatorname{SSA}\left(\mathfrak{m}, \mathfrak{m}^{\prime}\right) ; \square$-irreducibility . . . . . . . . . 703

A.M. was partially funded by Grant P12-FQM-2696.

$\triangle$ Alberto Mínguez

alberto.minguez@univie.ac.at

Erez Lapid

erez.m.lapid@gmail.com

1 Department of Mathematics, Weizmann Institute of Science, Rehovot 7610001, Israel

2 Department of Mathematics, University of Vienna, Oskar-Morgenstern-Platz 1, 1090 Vienna, Austria 
4 A geometric condition of Geiss-Leclerc-Schröer . . . . . . . . . . . . . . . . 707

5 Conjectural geometric conditions for $\mathrm{SA}\left(\mathfrak{m}, \mathfrak{m}^{\prime}\right) \ldots \ldots \ldots \ldots 710 \ldots \ldots$

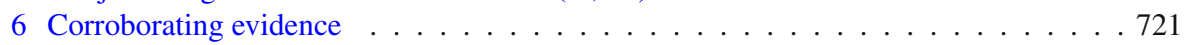

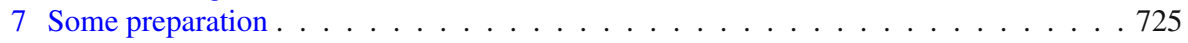

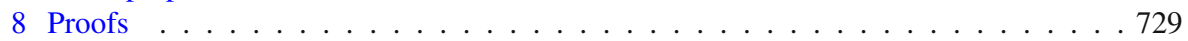

References . . . . . . . . . . . . . . . . . . . . 746

\section{Introduction}

Let $F$ be a local non-archimedean field. The smooth, complex representations of $\mathrm{GL}_{n}(F), n \geq 0$ were studied in depth in the seminal work of Bernstein and Zelevinsky [4,5,31]. In particular, Zelevinsky obtained a classification of the set Irr of irreducible representations of $\mathrm{GL}_{n}(F), n \geq 0$ in terms of multisegments - an essentially combinatorial object. Denote by $Z(\mathfrak{m})$ the irreducible representation corresponding to a multisegment $\mathfrak{m}$. A basic property is that for any $\pi_{i}=Z\left(\mathfrak{m}_{i}\right), i=1, \ldots, k$, the representation $Z\left(\mathfrak{m}_{1}+\cdots+\mathfrak{m}_{k}\right)$ occurs with multiplicity one in the Jordan-Hölder sequence of the representation $\pi_{1} \times \cdots \times \pi_{k}$ parabolically induced from $\pi_{1} \otimes \cdots \otimes \pi_{k}$. In particular, if $\pi_{1} \times \cdots \times \pi_{k}$ happens to be irreducible, then it is equivalent to $Z\left(\mathfrak{m}_{1}+\cdots+\mathfrak{m}_{k}\right)$. However, the problem of characterizing the irreducibility of $Z\left(\mathfrak{m}_{1}\right) \times \cdots \times Z\left(\mathfrak{m}_{k}\right)$ was left open. In this paper we study the case $k=2$.

An important special case is when $\pi_{2}=\pi_{1}$. We say that a representation $\pi$ is $\square$-irreducible if $\pi \times \pi$ is irreducible. It is a well-known consequence of a theorem of Bernstein [7] that every unitarizable representation $\pi \in \operatorname{Irr}$ is $\square$-irreducible. However, our emphasis in this paper is on general irreducible representations. The first example of a non- $\square$-irreducible $\pi \in \operatorname{Irr}$ was given by Leclerc [22].

The analogues of $\square$-irreducible representations in different (but related) contexts (where a different terminology is used) play an important role in the monoidal categorification of cluster algebras, a problem considered by Hernandez-Leclerc $[11,12]$ and more recently by Kang-Kashiwara-Kim-Oh [15]. We will not say anything about this problem here except to mention that the argument of [14], adapted to the case at hand, shows that if $\pi$ is $\square$ irreducible, then for any $\pi^{\prime} \in \operatorname{Irr}$, the $\operatorname{socles} \operatorname{soc}\left(\pi \times \pi^{\prime}\right)$ and $\operatorname{soc}\left(\pi^{\prime} \times \pi\right)$ are irreducible and each one occurs with multiplicity one in the Jordan-Hölder sequence of $\pi \times \pi^{\prime}$ [20]. It follows that if at least one of $\pi=Z(\mathfrak{m})$ and $\pi^{\prime}=Z\left(\mathfrak{m}^{\prime}\right)$ is $\square$-irreducible, then the irreducibility of $Z(\mathfrak{m}) \times Z\left(\mathfrak{m}^{\prime}\right)$ is equivalent to the conjunction of the condition

$$
Z\left(\mathfrak{m}+\mathfrak{m}^{\prime}\right) \hookrightarrow Z(\mathfrak{m}) \times Z\left(\mathfrak{m}^{\prime}\right)
$$

and its symmetric counterpart (interchanging $\mathfrak{m}$ and $\mathfrak{m}^{\prime}$ ). 
On the other hand, in [29] Zelevinsky proposed a geometric framework for his classification. The purpose was twofold: to interpret multiplicities of irreducible representations in a standard module using Kazhdan-Lusztig theory, and to give a geometric interpretation of the Zelevinsky involution. The first goal was expounded in [30] and is best explained by the Arakawa-Suzuki functors [1]—see $[20, \S 10]$ and the references therein for more details. The second goal was accomplished by Mœglin-Waldspurger who also give a combinatorial description of the resulting involution on multisegments [24]. (The fact that the Zelevinsky involution preserves irreducible representations is a special case of a more general result proved independently by Aubert [2,3] and Schneider-Stuhler [26]—see also [6] for a more recent treatment).

In this geometric framework, the work of Geiss-Leclerc-Schröer [9] suggests a simple, conjectural geometric criterion for the $\square$-irreducibility of $Z(\mathfrak{m})$. More precisely, fix a finite-dimensional graded vector space $V$ over $\mathbb{C}$ and let $\mathbb{G}(V)$ be the group of grading preserving automorphisms of $V$. Then, the multisegments with supercuspidal support determined by the graded dimension of $V$, parameterize the finitely many $\mathbb{G}(V)$-orbits on the space of linear transformations $A: V \rightarrow V$ that are homogeneous of degree 1 (or alternatively, $-1)$. They also parameterize the irreducible components of the variety $\mathfrak{X}(V)$ of commuting pairs of linear transformation $A, B: V \rightarrow V$ of degree 1 and -1 respectively. Denote by $\mathfrak{C}_{\mathfrak{m}}$ irreducible component corresponding to $\mathfrak{m}$. The conjecture of [20], inspired by [10, Conjecture 18.1], is that $Z(\mathfrak{m})$ is $\square$-irreducible if and only if $\mathfrak{C}_{\mathfrak{m}}$ admits an open $\mathbb{G}(V)$-orbit. (See Sect. 4 for more details.) This condition can be explicated fairly concretely and can be efficiently checked, at least by a randomized algorithm — see [20] where also a special case of this conjecture was proved, and was related to singularities of Schubert varieties of type $A$.

The main goal of this paper is to propose and substantiate a conjectural geometric criterion for the irreducibility of $Z(\mathfrak{m}) \times Z\left(\mathfrak{m}^{\prime}\right)$, provided that at least one of $Z(\mathfrak{m})$ and $Z\left(\mathfrak{m}^{\prime}\right)$ is $\square$-irreducible. More precisely, suppose that $\mathfrak{C}_{\mathfrak{m}} \subset \mathfrak{X}(V)$ and $\mathfrak{C}_{\mathfrak{m}^{\prime}} \subset \mathfrak{X}\left(V^{\prime}\right)$. Then, $\mathfrak{C}_{\mathfrak{m}+\mathfrak{m}^{\prime}} \subset \mathfrak{X}\left(V \oplus V^{\prime}\right)$ and we can embed $\mathfrak{C}_{\mathfrak{m}} \times \mathfrak{C}_{\mathfrak{m}^{\prime}}$ diagonally in $\mathfrak{C}_{\mathfrak{m}+\mathfrak{m}^{\prime}}$.

Conjecture 1 Suppose that at least one of $Z(\mathfrak{m})$ and $Z\left(\mathfrak{m}^{\prime}\right)$ is $\square$-irreducible. Then,

$Z(\mathfrak{m}) \times Z\left(\mathfrak{m}^{\prime}\right)$ is irreducible $\Longleftrightarrow \mathbb{G}\left(V \oplus V^{\prime}\right) \cdot\left(\mathfrak{C}_{\mathfrak{m}} \times \mathfrak{C}_{\mathfrak{m}^{\prime}}\right)$ is dense in $\mathfrak{C}_{\mathfrak{m}+\mathfrak{m}^{\prime}}$

More generally, under the above assumption we give a conjectural geometric criterion for (1). (See Sect. 5 for more details.) Recall that $\mathfrak{C}_{\mathfrak{m}}$ comes with a distinguished $\mathbb{G}(V)$-invariant Zariski open subset $\mathfrak{C}_{\mathfrak{m}}^{\circ}$ (containing the interior of $\mathfrak{C}_{\mathfrak{m}}$ in $\mathfrak{X}(V)$, but not equal to it in general). Let $\mathfrak{Y}$ be the subvariety of $\mathfrak{C}_{\mathfrak{m}+\mathfrak{m}^{\prime}}$ consisting of the pairs $(A, B)$ satisfying the following two conditions. 
1. $A\left(V^{\prime}\right), B\left(V^{\prime}\right) \subset V^{\prime}$, and $\left(\left.A\right|_{V^{\prime}},\left.B\right|_{V^{\prime}}\right) \in \mathfrak{C}_{\mathfrak{m}^{\prime}}^{\circ}$

2. The induced pair on the quotient $V$ belongs to $\mathfrak{C}_{\mathfrak{m}}^{\circ}$.

Conjecture 2 Suppose that at least one of $Z(\mathfrak{m})$ and $Z\left(\mathfrak{m}^{\prime}\right)$ is $\square$-irreducible. Then,

$$
Z\left(\mathfrak{m}+\mathfrak{m}^{\prime}\right) \hookrightarrow Z(\mathfrak{m}) \times Z\left(\mathfrak{m}^{\prime}\right) \Longleftrightarrow \mathbb{G}\left(V \oplus V^{\prime}\right) \cdot \mathfrak{Y} \text { is dense in } \mathfrak{C}_{\mathfrak{m}+\mathfrak{m}^{\prime}} .
$$

This conjecture implies Conjecture 1.

The geometric conditions in the two conjectures are in the spirit of the GeissLeclerc-Schröer condition. They are equally concrete and can be checked (by a randomized algorithm) very efficiently on a computer.

We will give supportive evidence for Conjecture 2, ergo, Conjecture 1. (See Sect. 6 for more details.) For instance, we show that they hold (without restriction on $\mathfrak{m}^{\prime}$ ) in any one of the following cases.

1. $Z(\mathfrak{m})$ is unitarizable.

2. $\mathfrak{m}$ is a ladder multisegment in the sense of [18].

3. $Z(\mathfrak{m})$ is generic, or more generally every segment in $\mathfrak{m}$ has the same length.

4. $Z(\mathfrak{m})$ is unramified, or more generally, the Zelevinsky dual of $Z(\mathfrak{m})$ is generic.

A key ingredient for the proof is the results of [19]. In fact, the conjecture was forged as an attempt to explain the results of [19] geometrically. We also show that Conjecture 2 satisfies a number of non-trivial consistency checks. While in all likelihood new ideas will be needed to establish the conjecture in general, we believe that the attestation that we already have so far cannot be coincidental.

In the best-case scenario, Conjecture 2 may in fact hold without restriction on $\mathfrak{m}$ and $\mathfrak{m}^{\prime}$. If true, this would imply that

$$
\begin{aligned}
& Z\left(\mathfrak{m}+\mathfrak{m}^{\prime}\right) \text { is a direct summand of } Z(\mathfrak{m}) \times Z\left(\mathfrak{m}^{\prime}\right) \Longleftrightarrow \\
& \mathbb{G}\left(V \oplus V^{\prime}\right) \cdot\left(\mathfrak{C}_{\mathfrak{m}} \times \mathfrak{C}_{\mathfrak{m}^{\prime}}\right) \text { is dense in } \mathfrak{C}_{\mathfrak{m}+\mathfrak{m}^{\prime}} .
\end{aligned}
$$

However, we feel that at this stage it would be too parlous to postulate such a strong form of Conjecture 2 (even in one direction) since our evidence for this generality is indirect and nowhere near conclusive. The main reason is that we do not have a practical way to check the condition (1) independently in general.

At any rate, the condition that $Z\left(\mathfrak{m}+\mathfrak{m}^{\prime}\right)$ is a direct summand of $Z(\mathfrak{m}) \times$ $Z\left(\mathfrak{m}^{\prime}\right)$ is strictly weaker than the irreducibility of $Z(\mathfrak{m}) \times Z\left(\mathfrak{m}^{\prime}\right)$. In fact, there are plenty of examples of irreducible representations $\pi$ of $\mathrm{GL}_{n}(F)$ such that $\pi \times \pi$ is semisimple of length two. Consequently, Conjecture 1 would have been false had we lifted the assumptions on $\mathfrak{m}$ and $\mathfrak{m}^{\prime}$. In order to obtain a 
precise irreducibility criterion without restriction on $\mathfrak{m}$ and $\mathfrak{m}^{\prime}$, we would need to characterize the condition

$$
Z\left(\mathfrak{m}+\mathfrak{m}^{\prime}\right)=\operatorname{soc}\left(Z(\mathfrak{m}) \times Z\left(\mathfrak{m}^{\prime}\right)\right)
$$

(which in general is stronger than (1)) and at present we do not have a conjectural geometric criterion for this.

In a different direction, a natural follow-up question, which we hope to address in the future, is to obtain a geometric insight on $\operatorname{soc}\left(Z(\mathfrak{m}) \times Z\left(\mathfrak{m}^{\prime}\right)\right)$, assuming as before that at least one of $Z(\mathfrak{m})$ and $Z\left(\mathfrak{m}^{\prime}\right)$ is $\square$-irreducible.

Finally, we point out that our conjectures do not seem to lie in the scope of the Langlands program.

The contents of the paper are as follows. After introducing the relevant notation and the Zelevinsky classification (Sect. 2) we recall the notion of $\square$-irreducible representations and basic facts about irreducibility of parabolic induction (Sect. 3). In Sect. 4 we recall the geometric condition of GeissLeclerc-Schröer and the conjecture relating it to $\square$-irreducibility. The heart of the paper is Sect. 5 where we state Conjectures 1 and 2 and analyze the pertinent geometric conditions. In Sect. 6 we state results confirming these conjectures in special cases and provide several consistency checks for them. These results are proved in Sect. 8 using Jacquet module techniques and other combinatorial tools which are recalled in Sect. 7.

\section{Notation and preliminaries}

\subsection{General notation}

Throughout the paper we fix a non-archimedean local field $F$ with normalized absolute value $|\cdot|$. For a non-negative integer $n$ let $\mathcal{M}_{n}$ denote the $\mathbb{C}$-linear, locally finite, abelian category of complex, smooth, finite length (hence admissible) representations of the group $\mathrm{GL}_{n}(F)$. Set

$$
\mathcal{M}=\oplus_{n \geq 0} \mathcal{M}_{n}
$$

and denote the simple objects of $\mathcal{M}$ by

$$
\operatorname{Irr}=\coprod_{n \geq 0} \operatorname{Irr} \mathcal{M}_{n} .
$$

In particular, write $\operatorname{Irr} \mathcal{M}_{0}=\{\mathbf{1}\}$. We denote by

$$
\operatorname{Irr}_{c}=\coprod_{n>0} \operatorname{Irr}_{c} \mathcal{M}_{n}
$$


the subset of irreducible supercuspidal representations. By abuse of notation we often write $\pi \in \mathcal{M}$ to mean that $\pi$ is an object of $\mathcal{M}$.

For $\tau, \pi \in \mathcal{M}$ we write $\tau \hookrightarrow \pi$ (resp., $\tau \rightarrow \pi$ ) if there exists an injective (resp., surjective) morphism from $\tau$ to $\pi$. If $\tau \in$ Irr and $\pi \in \mathcal{M}$, we will write $\tau \leq \pi$ for the condition that $\tau$ occurs as a subquotient of $\pi$ (i.e., $\tau$ occurs in the Jordan-Hölder sequence $\mathrm{JH}(\pi)$ of $\pi$ ). If $\tau$ occurs with multiplicity one in $\mathrm{JH}(\pi)$, then we will write $\tau \unlhd \pi$.

As customary, normalized parabolic induction with respect to standard (block upper triangular) parabolic subgroup will be denoted by $\times$. This is a bilinear biexact bifunctor with associativity constraints given by induction in stages. In other words, $\times$ endows $\mathcal{M}$ with the structure of a ring category with unit element 1 . The Grothendieck group

$$
\mathcal{R}=\oplus_{n \geq 0} \mathcal{R}_{n}
$$

of $\mathcal{M}$ inherits a structure of a graded commutative ring.

For any $\pi \in \mathcal{M}_{n}$ and a character $\chi$ of $F^{*}$, we denote by $\pi \cdot \chi$ the representation obtained from $\pi$ by twisting by the character $\chi \circ$ det. In particular, we write

$$
\vec{\pi}=\pi \cdot|\cdot|, \quad \overleftarrow{\pi}=\pi \cdot|\cdot|^{-1}
$$

We denote by $\pi^{\vee}$ the contragredient of $\pi$ and by $\operatorname{soc}(\pi)$ the socle of $\pi$, i.e., the largest semisimple subobject of $\pi$. (If $\pi \neq 0$, then $\operatorname{soc}(\pi) \neq 0$.)

Definition 1 We say that $\pi \in \mathcal{M}$ is socle irreducible (SI) if $\operatorname{soc}(\pi)$ is irreducible and $\operatorname{soc}(\pi) \unlhd \pi$.

Note that if $\pi \in \mathcal{M}$ is SI and $0 \neq \tau \hookrightarrow \pi$ then $\tau$ is SI and $\operatorname{soc}(\tau)=\operatorname{soc}(\pi)$. By the argument of [24, p. 173], for any $\pi_{1}, \pi_{2}, \tau \in$ Irr we have

$$
\tau \hookrightarrow \pi_{1} \times \pi_{2} \Longleftrightarrow \pi_{2} \times \pi_{1} \rightarrow \tau .
$$

For any set $A$ we denote by $\mathbb{N}(A)$ the free commutative monoid generated by the elements of $A$. It consists of finite formal sums of elements of $A$. The standard order on $\mathbb{N}(A)$ will be denoted by $\leq$.

We may view JH as a map from the objects of $\mathcal{M}$ to $\mathbb{N}$ (Irr). Thus, for $\tau \in \operatorname{Irr}$ and $\pi \in \mathcal{M}$ we have $\tau \leq \pi$ if and only if $\tau \leq \mathrm{JH}(\pi)$ in $\mathbb{N}$ (Irr).

For any $\pi \in \operatorname{Irr}$ there exist $\rho_{1}, \ldots, \rho_{k} \in \operatorname{Irr}_{c}$ such that $\pi \leq \rho_{1} \times \cdots \times \rho_{k}$. The supercuspidal support map

$$
\mathfrak{s}: \operatorname{Irr} \rightarrow \mathbb{N}\left(\operatorname{Irr}_{c}\right), \quad \pi \mapsto \rho_{1}+\cdots+\rho_{k}
$$

is well-defined and finite-to-one [5]. 


\subsection{Zelevinsky classification and involution [31]}

A segment (of length $k$ ) is a nonempty finite set of the form

$$
\Delta=\left\{\rho_{1}, \ldots, \rho_{k}\right\}
$$

where $\rho_{i} \in \operatorname{Irr}_{c}$ and $\rho_{i+1}=\vec{\rho}_{i}$ for all $i=1, \ldots, k-1$. For any such $\Delta$ we set

$$
\begin{gathered}
Z(\Delta):=\operatorname{soc}\left(\rho_{1} \times \cdots \times \rho_{k}\right) \in \operatorname{Irr}, \quad L(\Delta):=\operatorname{soc}\left(\rho_{k} \times \cdots \times \rho_{1}\right) \in \operatorname{Irr}, \\
\operatorname{supp}(\Delta)=\rho_{1}+\cdots+\rho_{k} \in \mathbb{N}\left(\operatorname{Irr}_{c}\right), \quad b(\Delta)=\rho_{1}, \quad e(\Delta)=\rho_{k}, \\
\Delta^{\vee}=\left\{\rho_{k}^{\vee}, \ldots, \rho_{1}^{\vee}\right\}, \vec{\Delta}=\left\{\vec{\rho}_{1}, \ldots, \vec{\rho}_{k}\right\}, \overleftarrow{\Delta}=\left\{\overleftarrow{\rho}_{1}, \ldots, \overleftarrow{\rho}_{k}\right\} .
\end{gathered}
$$

For compatibility, we also set $Z(\emptyset)=L(\emptyset)=1$. Let $\Sigma$ be the set of all segments. For $\Delta, \Delta^{\prime} \in \Sigma$ we write $\Delta \prec \Delta^{\prime}$ (and say that $\Delta$ precedes $\Delta^{\prime}$ ) if $b(\Delta) \notin \Delta^{\prime}, b\left(\Delta^{\prime}\right) \in \vec{\Delta}$ and $e\left(\Delta^{\prime}\right) \notin \Delta$. Thus,

$$
\overleftarrow{\Delta} \prec \Delta^{\prime} \Longleftrightarrow \Delta \prec \overrightarrow{\Delta^{\prime}} \Longleftrightarrow b\left(\Delta^{\prime}\right) \in \Delta \text { and } e(\Delta) \in \Delta^{\prime}
$$

We say that $\Delta, \Delta^{\prime} \in \Sigma$ are linked if either $\Delta \prec \Delta^{\prime}$ or $\Delta^{\prime} \prec \Delta$. (Equivalently, $\Delta \cup \Delta^{\prime} \in \Sigma$ and $\Delta, \Delta^{\prime} \subsetneq \Delta \cup \Delta^{\prime}$.)

By definition, a multisegment is an element of $\mathbb{N}(\Sigma)$. We extend the maps $\Delta \mapsto \operatorname{supp}(\Delta)$ and $\Delta \mapsto \Delta^{\vee}$ to an additive map

$$
\operatorname{supp}: \mathbb{N}(\Sigma) \rightarrow \mathbb{N}\left(\operatorname{Irr}_{c}\right)
$$

and an additive involution $\mathfrak{m} \mapsto \mathfrak{m}^{\vee}$ on $\mathbb{N}(\Sigma)$, respectively. Given $\mathfrak{m}=\Delta_{1}+$ $\cdots+\Delta_{k} \in \mathbb{N}(\Sigma)$ we may enumerate the $\Delta_{i}$ 's (in possibly more than one way) such that $\Delta_{i} \nprec \Delta_{j}$ whenever $i<j$. Then, the representation $\mathfrak{z}(\mathfrak{m})=$ $Z\left(\Delta_{1}\right) \times \cdots \times Z\left(\Delta_{k}\right)$ is SI and up to equivalence, depends only on $\mathfrak{m}$. The main result of Zelevinsky is that the map

$$
\mathfrak{m} \mapsto Z(\mathfrak{m}):=\operatorname{soc}(\mathfrak{z}(\mathfrak{m}))
$$

is a bijection between $\mathbb{N}(\Sigma)$ and Irr. There is also a dual bijection given by $\mathfrak{m} \mapsto L(\mathfrak{m}):=\operatorname{soc}(\lambda(\mathfrak{m}))$ where $\lambda(\mathfrak{m})=L\left(\Delta_{k}\right) \times \cdots \times L\left(\Delta_{1}\right)$ (under the same assumption on the order of the $\Delta_{i}$ 's). The latter is essentially the Langlands classification in this context. We refer the reader to [19, §3] for a summary of the basic properties of these bijections. In particular, $Z(0)=$ $L(0)=\mathbf{1}$,

$$
Z(\mathfrak{m})^{\vee}=Z\left(\mathfrak{m}^{\vee}\right) \text { and } L(\mathfrak{m})^{\vee}=L\left(\mathfrak{m}^{\vee}\right) \text { for any } \mathfrak{m} \in \mathbb{N}(\Sigma),
$$


$\mathfrak{s}(Z(\mathfrak{m}))=\mathfrak{s}(L(\mathfrak{m}))=\operatorname{supp} \mathfrak{m}$ and

$$
Z\left(\mathfrak{m}+\mathfrak{m}^{\prime}\right) \unlhd Z(\mathfrak{m}) \times Z\left(\mathfrak{m}^{\prime}\right) \text { for any } \mathfrak{m}, \mathfrak{m}^{\prime} \in \mathbb{N}(\Sigma)
$$

The two bijections are related by Deligne-Lusztig type duality. Let ${ }^{t}$ denote the duality functor on $\mathcal{M}$ defined by Schneider-Stuhler in [26], composed with the contragredient (in order to make it covariant). (See also [6] for a more recent approach.) Then

$$
Z(\mathfrak{m})^{t}=L(\mathfrak{m})
$$

for all $\mathfrak{m} \in \mathbb{N}(\Sigma)$ and

$$
\left(\pi \times \pi^{\prime}\right)^{t}=\pi^{\prime t} \times \pi^{t} \text { for any } \pi, \pi^{\prime} \in \mathcal{M} .
$$

Finally, let

$$
\#: \mathbb{N}(\Sigma) \rightarrow \mathbb{N}(\Sigma)
$$

be the bijection (in fact, involution) such that $L(\mathfrak{m})=Z\left(\mathfrak{m}^{\#}\right)$. Note that ${ }^{\#}$ is not additive.

We recall the recursive algorithm, due to Mœglin-Waldspurger [24], for the computation of the involution $\mathfrak{m} \mapsto \mathfrak{m}^{\#}$. For convenience (although it is not absolutely necessary) we fix a total order $\leq$ on $\operatorname{Irr}_{c}$ subject only to the condition that $\rho<\vec{\rho}$ for all $\rho \in \operatorname{Irr}_{c}$. Set $0^{\#}=0$. For $0 \neq \mathfrak{m}=\sum_{i \in I} \Delta_{i} \in \mathbb{N}(\Sigma)$ define

$$
\max \mathfrak{m}=\max _{i \in I} e\left(\Delta_{i}\right)
$$

Let $m \geq 1$ and $i_{1}, \ldots, i_{m} \in I$ be indices such that

$\max \mathfrak{m}=e\left(\Delta_{i_{1}}\right)$ and $b\left(\Delta_{i_{1}}\right)$ is maximal with respect to this property (8a) $\Delta_{i_{j+1}} \prec \Delta_{i_{j}}, e\left(\Delta_{i_{j+1}}\right)=e\left(\overleftarrow{\Delta}_{i_{j}}\right)$ and $b\left(\Delta_{i_{j+1}}\right)$ is maximal with respect to these properties, $j=1, \ldots, m-1$,

There is no $i \in I$ such that $\Delta_{i} \prec \Delta_{i_{m}}$ and $e\left(\Delta_{i}\right)=e\left(\overleftarrow{\Delta}_{i_{m}}\right)$

We call $i_{1}, \ldots, i_{m}$ leading indices of $\mathfrak{m}$. (Note that $i_{1}, \ldots, i_{m}$ are not uniquely determined, but $m$ and $\Delta_{i_{1}}, \ldots, \Delta_{i_{m}}$ are.) Let $\Delta(\mathfrak{m})$ be the segment

$$
\Delta(\mathfrak{m})=\left\{e\left(\Delta_{i_{m}}\right), \ldots, e\left(\Delta_{i_{1}}\right)\right\}
$$


and set $^{1}$

$$
\mathfrak{m}^{-}=\sum_{i \in I} \Delta_{i}^{\prime} \text { where } \Delta_{i}^{\prime}= \begin{cases}\Delta_{i} \backslash\left\{e\left(\Delta_{i}\right)\right\} & \text { if } i \in\left\{i_{1}, \ldots, i_{m}\right\}, \\ \Delta_{i} & \text { otherwise, }\end{cases}
$$

so that $\operatorname{supp}(\mathfrak{m})=\operatorname{supp}\left(\mathfrak{m}^{-}\right)+\operatorname{supp}(\Delta(\mathfrak{m}))$. (We discard $\Delta_{i}^{\prime}$ if it is the empty set.) By $[24, \S \mathrm{II} .2], \mathfrak{m}^{\#}=\left(\mathfrak{m}^{-}\right)^{\#}+\Delta(\mathfrak{m})$. Moreover, $\Delta(\mathfrak{m})$ is the segment of $\mathfrak{m}^{\#}$ such that $e(\Delta(\mathfrak{m}))=\max \mathfrak{m}=\max \mathfrak{m}^{\#}$ and $b(\Delta(\mathfrak{m}))$ is minimal with respect to this property.

\section{The conditions $\operatorname{SA}\left(\mathfrak{m}, \mathfrak{m}^{\prime}\right)$ and $\operatorname{SSA}\left(\mathfrak{m}, \mathfrak{m}^{\prime}\right)$; $\square$-irreducibility}

\subsection{Subadditivity conditions}

Let $\mathfrak{m}, \mathfrak{m}^{\prime} \in \mathbb{N}(\Sigma)$. The following properties will be our main concern.

Definition 21 . We denote by $\operatorname{SA}\left(\mathfrak{m}, \mathfrak{m}^{\prime}\right)$ the following equivalent conditions (by (2), (6) and (7)).

(a) $Z\left(\mathfrak{m}+\mathfrak{m}^{\prime}\right) \hookrightarrow Z(\mathfrak{m}) \times Z\left(\mathfrak{m}^{\prime}\right)$.

(b) $L\left(\mathfrak{m}+\mathfrak{m}^{\prime}\right) \hookrightarrow L\left(\mathfrak{m}^{\prime}\right) \times L(\mathfrak{m})$.

(c) $Z\left(\mathfrak{m}^{\prime}\right) \times Z(\mathfrak{m}) \rightarrow Z\left(\mathfrak{m}+\mathfrak{m}^{\prime}\right)$.

(d) $L(\mathfrak{m}) \times L\left(\mathfrak{m}^{\prime}\right) \rightarrow L\left(\mathfrak{m}+\mathfrak{m}^{\prime}\right)$.

2. We denote by $\operatorname{SSA}\left(\mathfrak{m}, \mathfrak{m}^{\prime}\right)$ the following equivalent conditions (by (5) and $[19$, Lemma 4.2]).

(a) $Z\left(\mathfrak{m}+\mathfrak{m}^{\prime}\right) \simeq \operatorname{soc}\left(Z(\mathfrak{m}) \times Z\left(\mathfrak{m}^{\prime}\right)\right)$.

(b) $\forall \tau \in \operatorname{Irr}, \tau \hookrightarrow Z(\mathfrak{m}) \times Z\left(\mathfrak{m}^{\prime}\right) \Longrightarrow \tau=Z\left(\mathfrak{m}+\mathfrak{m}^{\prime}\right)$.

(c) $Z(\mathfrak{m}) \times Z\left(\mathfrak{m}^{\prime}\right) \hookrightarrow \mathfrak{z}\left(\mathfrak{m}+\mathfrak{m}^{\prime}\right)$.

(d) $L\left(\mathfrak{m}+\mathfrak{m}^{\prime}\right) \simeq \operatorname{soc}\left(L\left(\mathfrak{m}^{\prime}\right) \times L(\mathfrak{m})\right)$.

(e) $\forall \tau \in \operatorname{Irr}, \tau \hookrightarrow L\left(\mathfrak{m}^{\prime}\right) \times L(\mathfrak{m}) \Longrightarrow \tau=L\left(\mathfrak{m}+\mathfrak{m}^{\prime}\right)$.

(f) $\lambda\left(\mathfrak{m}+\mathfrak{m}^{\prime}\right) \rightarrow L(\mathfrak{m}) \times L\left(\mathfrak{m}^{\prime}\right)$.

(The notation $\operatorname{SA}\left(\mathfrak{m}, \mathfrak{m}^{\prime}\right)$ and $\operatorname{SSA}\left(\mathfrak{m}, \mathfrak{m}^{\prime}\right)$ stand for "(strongly) subrepresentation + additive").

Clearly, $\operatorname{SSA}\left(\mathfrak{m}, \mathfrak{m}^{\prime}\right) \Longrightarrow \operatorname{SA}\left(\mathfrak{m}, \mathfrak{m}^{\prime}\right)$, while

the conditions $\operatorname{SA}\left(\mathfrak{m}, \mathfrak{m}^{\prime}\right)$ and $\operatorname{SA}\left(\mathfrak{m}^{\prime \vee}, \mathfrak{m}^{\vee}\right)$ are equivalent

and similarly for $\operatorname{SSA}\left(\mathfrak{m}, \mathfrak{m}^{\prime}\right)$.

A simple sufficient condition for $\operatorname{SSA}\left(\mathfrak{m}, \mathfrak{m}^{\prime}\right)$ is the following (see e.g., [19, Proposition 3.5]).

$\operatorname{SSA}\left(\mathfrak{m}, \mathfrak{m}^{\prime}\right) \Longleftarrow \forall$ segment $\Delta$ in $\mathfrak{m}$ and $\Delta^{\prime}$ in $\mathfrak{m}^{\prime}$ we have $\Delta \nprec \Delta^{\prime}$. (10)

\footnotetext{
$\overline{1}$ The notation $\mathfrak{m}^{-}$should not be confused with the same notation in [31].
} 
The conditions $\operatorname{SA}\left(\mathfrak{m}, \mathfrak{m}^{\prime}\right)$ and $\operatorname{SSA}\left(\mathfrak{m}, \mathfrak{m}^{\prime}\right)$ are instrumental for proving irreducibility. More precisely, again by (5),

$$
\begin{aligned}
& Z\left(\mathfrak{m}+\mathfrak{m}^{\prime}\right) \text { is a direct summand of } Z(\mathfrak{m}) \times Z\left(\mathfrak{m}^{\prime}\right) \Longleftrightarrow \\
& \qquad \operatorname{SA}\left(\mathfrak{m}, \mathfrak{m}^{\prime}\right) \text { and } \operatorname{SA}\left(\mathfrak{m}^{\prime}, \mathfrak{m}\right) \\
& Z \begin{array}{l}
Z(\mathfrak{m}) \times Z\left(\mathfrak{m}^{\prime}\right) \text { is irreducible } \Longleftrightarrow Z\left(\mathfrak{m}+\mathfrak{m}^{\prime}\right) \simeq Z(\mathfrak{m}) \times Z\left(\mathfrak{m}^{\prime}\right) \\
\Longleftrightarrow \operatorname{SSA}\left(\mathfrak{m}, \mathfrak{m}^{\prime}\right) \text { and } \operatorname{SSA}\left(\mathfrak{m}^{\prime}, \mathfrak{m}\right) \Longleftrightarrow \operatorname{SA}\left(\mathfrak{m}, \mathfrak{m}^{\prime}\right) \text { and } \operatorname{SSA}\left(\mathfrak{m}^{\prime}, \mathfrak{m}\right) .
\end{array}
\end{aligned}
$$

As we will recall below, it is possible that $Z(\mathfrak{m}) \times Z\left(\mathfrak{m}^{\prime}\right)$ is semisimple but not irreducible. In particular, $\operatorname{SSA}\left(\mathfrak{m}, \mathfrak{m}^{\prime}\right)$ is strictly stronger than $\operatorname{SA}\left(\mathfrak{m}, \mathfrak{m}^{\prime}\right)$.

\section{2 $\square$-irreducible representations}

For any $0 \neq \pi_{i} \in \mathcal{M}_{n_{i}}, i=1,2$ consider the standard (unnormalized) intertwining operators

$$
\pi_{1} \cdot|\cdot|^{s} \times \pi_{2} \rightarrow \pi_{2} \times \pi_{1} \cdot|\cdot|^{s} .
$$

They are given by convergent integrals for $\Re s \gg 0$ and admit a meromorphic continuation (e.g., [28, Ch. IV]). ${ }^{2}$ The leading term in the Laurent expansion at $s=0$ is a non-zero intertwining operator

$$
R_{\pi_{1}, \pi_{2}}: \pi_{1} \times \pi_{2} \rightarrow \pi_{2} \times \pi_{1} .
$$

The following key definition is inspired by the work of Hernandez-Leclerc and Kang-Kashiwara-Kim-Oh.

Definition 3 (See $[14,15,20]$ ) An object $\pi \neq 0$ of $\mathcal{M}$ (necessarily in Irr) is called $\square$-irreducible if the following equivalent conditions are satisfied.

1. $\pi \times \pi$ is irreducible.

2. $\operatorname{End}_{\mathcal{M}}(\pi \times \pi)=\mathbb{C}$.

3. $R_{\pi, \pi}$ is a scalar.

4. $\pi \times \pi$ is SI.

5. $\pi=Z(\mathfrak{m})$ and $\operatorname{SSA}(\mathfrak{m}, \mathfrak{m})$.

We denote by $\operatorname{Irr}^{\square} \subset$ Irr the class of $\square$-irreducible representations. Clearly, this class is invariant under contragredient and the duality ${ }^{t}$. The first example

\footnotetext{
2 The intertwining operators depend implicitly on a choice of a Haar measure on the space of $n_{1} \times n_{2}$ matrices, so technically they are only defined up to a positive scalar. However, for our purposes this is immaterial.
} 
of an irreducible representation $\pi$ (of $\mathrm{GL}_{8}(F)$ ) which is not $\square$-irreducible was given by Leclerc [22]. Explicitly, let

$$
\pi=Z(\mathfrak{m}) \text { where } \mathfrak{m}=[1,2]+[-1,1]+[0,0]+[-2,-1],
$$

and for simplicity we write $[a, b]$ for the segment $\left\{|\cdot|^{a}, \ldots,|\cdot|^{b}\right\}$ consisting of characters of $\mathrm{GL}_{1}(F)=F^{*}$. Then,

$$
\begin{aligned}
& \pi \times \pi=Z(\mathfrak{m}+\mathfrak{m}) \oplus Z([1,2]+[0,1]+[-1,2]+[-1,0]+[-2,1]+[-2,-1]) \\
= & Z(\mathfrak{m}+\mathfrak{m}) \oplus(Z([-1,2]+[-2,1]) \times Z([1,2]+[0,1]+[-1,0]+[-2,-1])) .
\end{aligned}
$$

Note that $\pi^{\vee}=\pi^{t}=\pi$.

\subsection{Basic properties}

An important property of $\square$-irreducible representations is the following.

Proposition 1 ([20] which is based on [14,15]) Suppose that $\pi$ is $\square$ irreducible. Then, for any $\pi^{\prime} \in \mathrm{Irr}$,

1. $\pi \times \pi^{\prime}$ and $\pi^{\prime} \times \pi$ are $S I$.

2. $\operatorname{soc}\left(\pi \times \pi^{\prime}\right)=\operatorname{Im} R_{\pi^{\prime}, \pi}$.

3. $\operatorname{soc}\left(\pi^{\prime} \times \pi\right)=\operatorname{Im} R_{\pi, \pi^{\prime}}$.

4. The following conditions are equivalent:

(a) $\pi \times \pi^{\prime}$ is irreducible.

(b) $R_{\pi, \pi^{\prime}}$ is an isomorphism.

(c) $R_{\pi^{\prime}, \pi}$ is an isomorphism.

(d) $\pi \times \pi^{\prime} \simeq \pi^{\prime} \times \pi$.

(e) $\operatorname{soc}\left(\pi \times \pi^{\prime}\right) \simeq \operatorname{soc}\left(\pi^{\prime} \times \pi\right)$.

Corollary 1 Suppose that

at least one of $Z(\mathfrak{m})$ or $Z\left(\mathfrak{m}^{\prime}\right)$ is $\square$-irreducible.

(ALO)

Then, the following conditions are equivalent.

1. $\operatorname{SA}\left(\mathfrak{m}, \mathfrak{m}^{\prime}\right)$.

2. $\operatorname{SSA}\left(\mathfrak{m}, \mathfrak{m}^{\prime}\right)$.

3. The image of $R_{Z\left(\mathfrak{m}^{\prime}\right), Z(\mathfrak{m})}$ is isomorphic to $Z\left(\mathfrak{m}+\mathfrak{m}^{\prime}\right)$.

In particular, $Z(\mathfrak{m}) \times Z\left(\mathfrak{m}^{\prime}\right)$ is irreducible $\Longleftrightarrow$ both $\mathrm{SA}\left(\mathfrak{m}, \mathfrak{m}^{\prime}\right)$ and $\mathrm{SA}\left(\mathfrak{m}^{\prime}, \mathfrak{m}\right)$ are satisfied.

We do not know whether in general the condition $\mathrm{SA}\left(\mathfrak{m}, \mathfrak{m}^{\prime}\right)$ implies that $Z\left(\mathfrak{m}+\mathfrak{m}^{\prime}\right)$ is a subrepresentation of the image of $R_{Z\left(\mathfrak{m}^{\prime}\right), Z(\mathfrak{m})}$. 
Remark 1 Let $\pi=Z(\mathfrak{m}) \in \operatorname{Irr}$ and $\Pi=\pi \times \pi$.

1. By (2), every irreducible subrepresentation of $\Pi$ also occurs as a quotient of $\Pi$. Hence, any irreducible subrepresentation $\tau$ of $\Pi$ such that $\tau \unlhd \Pi$ is a direct summand of $\Pi$. It easily follows that if $\Pi$ is multiplicity free (i.e., if every element of $\mathrm{JH}(\Pi)$ occurs with multiplicity one), then $\Pi$ is semisimple, and in particular $\mathrm{SA}(\mathfrak{m}, \mathfrak{m})$ is satisfied.

2. In Leclerc's example (12), $\Pi$ is of length two, and in particular multiplicity free. Hence, in general the condition $\mathrm{SA}(\mathfrak{m}, \mathfrak{m})$ is strictly weaker than $\operatorname{SSA}(\mathfrak{m}, \mathfrak{m})$ (i.e., the $\square$-irreducibility of $\pi$ ).

3. The first-named author computed $\mathrm{JH}(\Pi)$ for all multisegments $\mathfrak{m}$ consisting of at most 6 segments. (The computation involves Kazhdan-Lusztig polynomials of the symmetric group $S_{12}$. See [17] for more details.) It turns out that in these cases, $\Pi$ is multiplicity free if and only if $\Pi$ has length one or two. An example where this condition fails is

$$
\mathfrak{m}=[1,3]+[-2,2]+[-1,1]+[0,0]+[-3,-1]
$$

in which $\Pi$ has length 9 with

$$
\begin{aligned}
& Z([-2,3]+[-3,2]+[1,3]+[-1,2]+[-2,1]+[0,1]+[-1,0]+[-3,-1]) \\
& \quad=Z([-2,3]+[-3,2]) \times Z([1,3]+[-1,2]+[-2,1]+[0,1]+[-1,0]+[-3,-1])
\end{aligned}
$$

occurring with multiplicity 2 in $\mathrm{JH}(\Pi)$. Another example is

$$
\mathfrak{m}=[2,4]+[-2,3]+[-1,2]+[0,1]+[-4,0]+[-3,-1]
$$

for which $\Pi$ has length 257 (the largest possible for $\mathfrak{m}$ up to 6 segments), with

$$
\begin{aligned}
& Z([-2,4]+[-3,3]+[-4,2]+[2,4]+[-4,3]+[-1,2]+[-2,1] \\
& +[-3,0]+[0,0]+[-1,-1])=Z([-2,4]+[-3,3]+[-4,2]) \times \\
& Z([2,4]+[-4,3]+[-1,2]+[-2,1]+[-3,0]) \times Z([0,0]+[-1,-1])
\end{aligned}
$$

occurring with multiplicity 9 (again, the highest possible) in $\mathrm{JH}(\Pi)$.

4. Unfortunately, in contrast to the multiplicity freeness of $\Pi$, we do not have a practical way to completely determine whether $\Pi$ is semisimple or whether the condition $\mathrm{SA}(\mathfrak{m}, \mathfrak{m})$ is satisfied. In fact, at the moment we are unable to refute the condition $\mathrm{SA}(\mathfrak{m}, \mathfrak{m})$, or even the semisimplicity of $\Pi$, in any single example.

We also remark that if $\pi_{1}, \pi_{2}$ are $\square$-irreducible and $\pi_{1} \times \pi_{2}$ is irreducible, then $\pi_{1} \times \pi_{2}$ is $\square$-irreducible ([20, Corollary 2.7]). 
Finally, we mention another simple property, which is a powerful tool for proving $\square$-irreducibility ([20, Lemma 2.10]).

Suppose that $\pi_{1}, \pi_{2} \in \operatorname{Irr}^{\square}, \pi \hookrightarrow \pi_{1} \times \pi_{2}$ and $\pi \times \pi_{1}$ is irreducible.

$$
\text { Then, } \pi \in \operatorname{Irr}^{\square} \text {. }
$$

An interesting question, which will not be discussed here, is whether conversely, given a non-supercuspidal $\pi \in \operatorname{Irr}^{\square}$, do there always exist $\mathbf{1} \neq \pi_{1}, \pi_{2} \in \operatorname{Irr}^{\square}$ such that $\pi \hookrightarrow \pi_{1} \times \pi_{2}$ and $\pi \times \pi_{1}$ is irreducible?

\section{A geometric condition of Geiss-Leclerc-Schröer}

\subsection{Zelevinsky's commuting varieties}

We recall Zelevinsky's geometric picture of his classification [16,24,29,30]. Consider a finite-dimensional $\operatorname{Irr}_{c}$-graded $\mathbb{C}$-vector space

$$
\mathbf{V}=\oplus_{\rho \in \operatorname{Irr}_{c}} \mathbf{V}_{\rho} .
$$

Up to isomorphism, $\mathbf{V}$ is determined by its graded dimension

$$
\operatorname{grdim} \mathbf{V}=\sum_{\rho \in \operatorname{Irr}_{c}}\left(\operatorname{dim} \mathbf{V}_{\rho}\right) \rho \in \mathbb{N}\left(\operatorname{Irr}_{c}\right)
$$

Let

$$
\mathbb{G}(\mathbf{V})=\prod_{\rho \in \operatorname{Irr}_{c}} \mathrm{GL}\left(\mathbf{V}_{\rho}\right)
$$

be the group of grading preserving linear automorphisms of $\mathbf{V}$ and let

$$
\overrightarrow{\mathbb{E}}(\mathbf{V})(\text { resp., } \overleftarrow{\mathbb{E}}(\mathbf{V}))
$$

be the vector space of (nilpotent) linear transformations $A: \mathbf{V} \rightarrow \mathbf{V}$ such that $A\left(\mathbf{V}_{\rho}\right) \subseteq \mathbf{V}_{\vec{\rho}}$ (resp., $A\left(\mathbf{V}_{\rho}\right) \subseteq \mathbf{V}_{\overleftarrow{\rho}}$ ) for all $\rho \in \operatorname{Irr}_{c}$. We will use the notational convention $\overleftrightarrow{\mathbb{E}}(\mathbf{V})$ to denote either of the spaces $\overleftrightarrow{\mathbb{E}}(\mathbf{V})$ and $\overleftarrow{\mathbb{E}}(\mathbf{V})$. A similar praxis will apply in other instances.

The group $\mathbb{G}(\mathbf{V})$ acts on each of the spaces $\stackrel{\leftrightarrow}{\mathbb{E}}(\mathbf{V})$ (by conjugation) with finitely many orbits and the spaces $\overleftrightarrow{\mathbb{E}}(\mathbf{V})$ are in duality with respect to the $\mathbb{G}(\mathbf{V})$-invariant pairing $(A, B) \mapsto \operatorname{tr} A B=\operatorname{tr} B A$. The orbit of an element 
$A \in \overleftrightarrow{\mathbb{E}}(\mathbf{V})$ is determined by the non-negative integers rk $\left.A^{i}\right|_{\mathbf{V}_{\rho}}, i \geq 0, \rho \in \operatorname{Irr}_{c}$ (which are non-zero for only finitely many $i$ 's and $\rho$ 's). The orbits are parameterized by the multisegments $\mathfrak{m}$ such that $\operatorname{supp}(\mathfrak{m})=\operatorname{grdim} \mathbf{V}$. Concretely, given such $\mathfrak{m}=\sum_{i \in I} \Delta_{i} \in \mathbb{N}(\Sigma)$, an element $A \in \overleftrightarrow{\mathbb{E}}(\mathbf{V})$ belongs to the orbit $\overleftrightarrow{\mathcal{O}}_{\mathfrak{m}}$ corresponding to $\mathfrak{m}$ if and only if there exists a graded basis $x_{\rho, i}, i \in I$, $\rho \in \Delta_{i}$ (graded by $\rho$ ) of $\mathbf{V}$ for which $A$ has a graded Jordan normal form

$$
A x_{\rho, i}=x_{\stackrel{\leftrightarrow}{\rho}, i}
$$

where we set $x_{\rho, j}=0$ if $\rho \notin \Delta_{j}$.

The $\mathbb{G}(\mathbf{V})$-orbits in $\overleftrightarrow{\mathbb{E}}(\mathbf{V})$ are also in one-to-one correspondence with the irreducible components of the commuting variety

$$
\mathfrak{X}(\mathbf{V})=\{(A, B) \in \overrightarrow{\mathbb{E}}(\mathbf{V}) \times \overleftarrow{\mathbb{E}}(\mathbf{V}): A B=B A\}
$$

[25]. These bijections take an orbit $\mathcal{O}$ in $\overleftrightarrow{\mathbb{E}}(\mathbf{V})$ to the Zariski closure $\stackrel{\leftrightarrow}{p}_{\mathbf{V}}^{-1}(\mathcal{O})$ of $\stackrel{\leftrightarrow}{p}_{\mathbf{V}}^{-1}(\mathcal{O})$ where

$$
\stackrel{\leftrightarrow}{p}_{\mathbf{V}}: \mathfrak{X}(\mathbf{V}) \rightarrow \overleftrightarrow{\mathbb{E}}(\mathbf{V})
$$

are the canonical projections. Let $\stackrel{\mathfrak{C}}{\mathfrak{m}}_{\mathfrak{m}}$ be the irreducible components of $\mathfrak{X}(\mathbf{V})$ corresponding to $\overleftrightarrow{\mathcal{O}}_{\mathfrak{m}}$. They are related by $\overrightarrow{\mathfrak{C}}_{\mathfrak{m}}=\overleftarrow{\mathfrak{C}}_{\mathfrak{m}^{*}}$ [24] where we recall that $\mathfrak{m}^{\#}$ was defined in Sect. 2.2. We also write

$$
\stackrel{\leftrightarrow}{\mathfrak{C}}_{\mathfrak{m}}^{\circ}=\stackrel{\leftrightarrow}{p}_{\mathbf{V}}^{-1}\left(\overleftrightarrow{\mathcal{O}}_{\mathfrak{m}}\right)
$$

so that $\stackrel{\leftrightarrow}{\mathfrak{C}}_{\mathfrak{m}}={\stackrel{\leftrightarrow}{\mathfrak{C}_{\mathfrak{m}}^{\circ}}}_{\text {. Note that } \stackrel{\leftrightarrow}{\mathfrak{C}}_{\mathfrak{m}}^{\circ}}$ is $\mathbb{G}(\mathbf{V})$-invariant and open in $\stackrel{\leftrightarrow}{\mathfrak{C}}_{\mathfrak{m}}$. (We caution that $\overleftrightarrow{\mathfrak{C}}_{\mathfrak{m}}^{\circ}$ contains the interior $\overleftrightarrow{\mathfrak{C}}_{\mathfrak{m}} \backslash \cup_{\mathfrak{m}^{\prime} \neq \mathfrak{m}} \overleftrightarrow{\mathfrak{C}}_{\mathfrak{m}^{\prime}}$ of $\overleftrightarrow{\mathfrak{C}}_{\mathfrak{m}}$ in $\mathfrak{X}(\mathbf{V})$, but the inclusion is strict in general).

\subsection{The condition $G L S(\mathrm{~m})$}

In general, there are infinitely many $\mathbb{G}(\mathbf{V})$-orbits in $\mathfrak{X}(\mathbf{V})$. Following GeissLeclerc-Schröer [10] we make the following definition. (As before, $\mathfrak{m} \in \mathbb{N}(\Sigma)$ and $\operatorname{supp} \mathfrak{m}=\operatorname{grdim} \mathbf{V}$.)

Definition 4 We say that the condition $G L S(\mathfrak{m})$ holds if $\overrightarrow{\mathfrak{C}}_{\mathfrak{m}}$ admits an open (i.e., dense) $\mathbb{G}(\mathbf{V})$-orbit. 
By [20, Remark 4.6], the analogous condition for $\overleftarrow{\mathfrak{C}}_{\mathfrak{m}}$ is equivalent. Therefore, from now on we will exclusively work with $\overrightarrow{\mathfrak{C}}_{\mathfrak{m}}$. In order to simplify the notation, we will henceforth write $\mathfrak{C}_{\mathfrak{m}}$ instead of $\overrightarrow{\mathfrak{C}}_{\mathfrak{m}}$ (and similarly for $\mathcal{O}_{\mathfrak{m}}$ and $\mathfrak{C}_{\mathfrak{m}}^{\circ}$ ).

Note that an open $\mathbb{G}(\mathbf{V})$-orbit in $\mathfrak{C}_{\mathfrak{m}}$, if exists, is necessarily contained in $\mathfrak{C}_{\mathfrak{m}}^{\circ}$.

Conjecture 3 ([20], following [10, Conjecture 18.1]) For any multisegment $\mathfrak{m}, Z(\mathfrak{m})$ is $\square$-irreducible if and only if $G L S(\mathfrak{m})$ holds.

\subsection{Linearization}

As in [20, §4], it is advantageous to explicate the condition $G L S(\mathfrak{m})$ by linearization. More precisely, fix $\mathbf{A}_{\mathfrak{m}} \in \mathcal{O}_{\mathfrak{m}}$ and write it in the form (14) for a suitable basis $x_{\rho, i}, \rho \in \Delta_{i}, i \in I$. The map $\overleftarrow{p}_{\mathbf{V}}$ identifies the fibre $\vec{p}_{\mathbf{V}}^{-1}\left(\mathbf{A}_{\mathfrak{m}}\right)$ with the centralizer $\mathbf{C}_{\mathfrak{m}}$ of $\mathbf{A}_{\mathfrak{m}}$ in $\overleftarrow{\mathbb{E}}(\mathbf{V})$. The stabilizer $\mathbf{G}_{\mathfrak{m}}$ of $\mathbf{A}_{\mathfrak{m}}$ in $\mathbb{G}(\mathbf{V})$ acts linearly on $\mathbf{C}_{\mathfrak{m}}$. (Note that $\mathbf{G}_{\mathfrak{m}}$ is usually not reductive.) Thus, $G L S(\mathfrak{m})$ holds if and only if $\mathbf{G}_{\mathfrak{m}}$ admits an open (i.e., dense) orbit in $\mathbf{C}_{\mathfrak{m}}$, i.e., $\mathbf{C}_{\mathfrak{m}}$ is a prehomogenuous vector space with respect to the action of $\mathbf{G}_{\mathfrak{m}}$. Passing to the Lie algebra, we can rephrase it by saying that there exists $\lambda \in \mathbf{C}_{\mathfrak{m}}$ such that $\left[\mathfrak{g}_{\mathfrak{m}}, \lambda\right]=\mathbf{C}_{\mathfrak{m}}$ where $\mathfrak{g}_{\mathfrak{m}}$ is the Lie algebra of $\mathbf{G}_{\mathfrak{m}}$.

The vector space $\mathbf{C}_{\mathfrak{m}}$ was explicated in [24, Lemme II.4] as follows. Let $X_{\mathfrak{m}}$ be the set

$$
X_{\mathfrak{m}}=\left\{(i, j) \in I \times I: \Delta_{i} \prec \Delta_{j}\right\} .
$$

Then, $\mathbf{C}_{\mathfrak{m}}$ admits a basis $\alpha_{i, j}=\alpha_{i, j}^{\mathfrak{m}},(i, j) \in X_{\mathfrak{m}}$ given by

$$
\alpha_{i, j}\left(x_{\rho, l}\right)=\delta_{j, l} x_{\stackrel{\leftarrow}{\rho}, i}, \quad \rho \in \Delta_{l}, l \in I
$$

where $\delta_{r, s}$ is Kronecker's delta. (Recall that by convention, $x_{\rho, j}=0$ if $\rho \notin$ $\Delta_{j}$.) For any $\lambda \in \mathbf{C}_{\mathfrak{m}}$, we write the coordinates of $\lambda$ with respect to the basis $\alpha_{i, j}$ as $\lambda_{i, j} \in \mathbb{C},(i, j) \in X_{\mathfrak{m}}$. Thus,

$$
\lambda\left(x_{\rho, j}\right)=\sum_{i \in I:(i, j) \in X_{\mathfrak{m}}} \lambda_{i, j} x_{\overleftarrow{\rho}, i}, \quad \rho \in \Delta_{j} .
$$

Similarly, the group $\mathbf{G}_{\mathfrak{m}}$ and its Lie algebra $\mathfrak{g}_{\mathfrak{m}}$ are described in [24, Lemme II.5]. Let $Y_{\mathfrak{m}}$ be the set

$$
Y_{\mathfrak{m}}=\left\{(i, j) \in I \times I: \Delta_{i} \prec \vec{\Delta}_{j}\right\} .
$$


Then, as a vector space, $\mathfrak{g}_{\mathfrak{m}}$ has a basis $\beta_{i, j}=\beta_{i, j}^{\mathfrak{m}},(i, j) \in Y_{\mathfrak{m}}$ given by

$$
\beta_{i, j}\left(x_{\rho, l}\right)=\delta_{j, l} x_{\rho, i}, \quad \rho \in \Delta_{l}, l \in I .
$$

Thus, $g \in \mathbb{G}(\mathbf{V})$ is in $\mathbf{G}_{\mathfrak{m}}$ if and only if

$$
g\left(x_{\rho, j}\right)=\sum_{i:(i, j) \in Y_{\mathfrak{m}}} g_{i, j} x_{\rho, i}, \quad \rho \in \Delta_{j}
$$

for scalars $g_{i, j},(i, j) \in Y_{\mathfrak{m}}$. We will call $g_{i, j}$ the coordinates of $g$.

Moreover, we have

$$
\left[\beta_{i, j}, \alpha_{k, l}\right]=\delta_{j, k} \alpha_{i, l}-\delta_{i, l} \alpha_{k, j}, \quad(i, j) \in Y_{\mathfrak{m}}, \quad(k, l) \in X_{\mathfrak{m}}
$$

where for convenience we set $\alpha_{r, s}=0$ if $(r, s) \notin X_{\mathfrak{m}}$.

Therefore, by passing to the dual map we can explicate the surjectivity of the linear map $[\cdot, \lambda]: \mathfrak{g}_{\mathfrak{m}} \rightarrow \mathbf{C}_{\mathfrak{m}}$ as follows.

Lemma 1 Consider the $\mathbb{C}$-vector space $\mathbb{C}^{Y_{\mathfrak{m}}}$ with standard basis $\left\{y_{i, j}\right.$ : $\left.(i, j) \in Y_{\mathfrak{m}}\right\}$. Then, $G L S(\mathfrak{m})$ is satisfied if and only if there exists $\lambda \in \mathbf{C}_{\mathfrak{m}}$, with coordinates $\lambda_{i, j} \in \mathbb{C},(i, j) \in X_{\mathfrak{m}}$, such that the vectors

$$
v_{i, j}^{\mathfrak{m}}(\lambda):=\sum_{k \in I:(k, j) \in X_{\mathfrak{m}},(i, k) \in Y_{\mathfrak{m}}} \lambda_{k, j} y_{i, k}-\sum_{l \in I:(l, j) \in Y_{\mathfrak{m}},(i, l) \in X_{\mathfrak{m}}} \lambda_{i, l} y_{l, j}, \quad(i, j) \in X_{\mathfrak{m}}
$$

are linearly independent in $\mathbb{C}^{Y_{\mathfrak{m}}}$.

Note that the linear independence of $v_{i, j}^{\mathfrak{m}}(\lambda),(i, j) \in X_{\mathfrak{m}}$ is an open and $\mathbf{G}_{\mathfrak{m}}$-invariant condition in $\lambda \in \mathbf{C}_{\mathfrak{m}}$. This condition is also easy to check (by a randomized algorithm) on a computer. (See [20, Remark 4.9] for a hypothetical approach to make this deterministic.) We refer to [20, §4] for examples and further discussion.

The condition $G L S(\mathfrak{m})$ is invariant under the involutions $\mathfrak{m} \mapsto \mathfrak{m}^{\vee}$ and $\mathfrak{m} \mapsto \mathfrak{m}^{\#}$ [20, Remark 4.18].

The main result of [20] is that Conjecture 3 holds in the special case where $\mathfrak{m}=\sum_{i \in I} \Delta_{i}$ is regular, i.e., when $e\left(\Delta_{i}\right) \neq e\left(\Delta_{j}\right)$ and $b\left(\Delta_{i}\right) \neq b\left(\Delta_{j}\right)$ for all $i \neq j$. In this case, the condition is also related, somewhat surprisingly, to smoothness of Schubert varieties of type $A$.

It was also verified computationally that Conjecture 3 holds for $\mathfrak{m}$ consisting of up to 6 segments.

\section{Conjectural geometric conditions for $\mathrm{SA}\left(\mathfrak{m}, \mathfrak{m}^{\prime}\right)$}

In this section, which is the heart of the paper, we introduce a new, and easy to check, geometric condition $\mathrm{SG}\left(\mathfrak{m}, \mathfrak{m}^{\prime}\right)$ pertaining to two multisegments $\mathfrak{m}, \mathfrak{m}^{\prime}$. 
This condition is in the spirit of the condition $G L S(\mathfrak{m})$ discussed in Sect. 4.2. We conjecture that $\operatorname{SG}\left(\mathfrak{m}, \mathfrak{m}^{\prime}\right)$ is equivalent to $\operatorname{SA}\left(\mathfrak{m}, \mathfrak{m}^{\prime}\right)$, at least under the condition (ALO) of Sect. 3.3. In particular, in this case it would give a practical condition for irreducibility of parabolic induction.

\subsection{General setup}

We continue to work with the geometric setup and the notation of the previous section.

Let $\mathbf{V}$ and $\mathbf{V}^{\prime}$ be two finite-dimensional $\operatorname{Irr}_{c}$-graded vector spaces over $\mathbb{C}$. Consider the direct sum $\tilde{\mathbf{V}}=\mathbf{V} \oplus \mathbf{V}^{\prime}$ and the short exact sequence

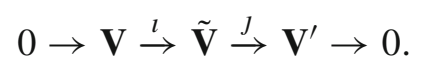

Define the spaces $\overrightarrow{\mathbb{E}^{l}}(\tilde{\mathbf{V}})$ and $\overleftarrow{\mathbb{E}^{l}}(\tilde{\mathbf{V}})$ by

$$
\overleftrightarrow{\mathbb{E}^{l}}(\tilde{\mathbf{V}})=\{A \in \overleftrightarrow{\mathbb{E}}(\tilde{\mathbf{V}}): \jmath \circ A \circ \imath=0\}=\{A \in \overleftrightarrow{\mathbb{E}}(\tilde{\mathbf{V}}): A(\mathbf{V}) \subset \mathbf{V}\}
$$

and let

$$
\mathfrak{X}^{l}(\tilde{\mathbf{V}})=\mathfrak{X}(\tilde{\mathbf{V}}) \cap\left(\overrightarrow{\mathbb{E}^{l}}(\tilde{\mathbf{V}}) \times \overleftrightarrow{\mathbb{E}^{l}}(\tilde{\mathbf{V}})\right)=\{(A, B) \in \mathfrak{X}(\tilde{\mathbf{V}}): A(\mathbf{V}), B(\mathbf{V}) \subset \mathbf{V}\}
$$

The stabilizer $\mathbb{P}^{l}$ of $\mathbf{V}$ in $\mathbb{G}(\tilde{\mathbf{V}})$ is a parabolic subgroup of $\mathbb{G}(\tilde{\mathbf{V}})$ with Levi quotient isomorphic to $\mathbb{G}(\mathbf{V}) \times \mathbb{G}\left(\mathbf{V}^{\prime}\right)$ and unipotent radical

$$
\mathbb{U}^{\imath}=\{g \in \mathbb{G}(\tilde{\mathbf{V}}): g \circ \imath=\imath \text { and } \jmath \circ g=\jmath\},
$$

which is commutative.

Any $A \in \overleftrightarrow{\mathbb{E}^{\prime}}(\tilde{\mathbf{V}})$ restricts to a map in $\overleftrightarrow{\mathbb{E}}(\mathbf{V})$ and induces a map in $\overleftrightarrow{\mathbb{E}}\left(\mathbf{V}^{\prime}\right)$. We denote by

$$
\overleftrightarrow{p}^{l}: \overleftrightarrow{\mathbb{E}^{l}}(\tilde{\mathbf{V}}) \rightarrow \overleftrightarrow{\mathbb{E}}(\mathbf{V}) \times \overleftrightarrow{\mathbb{E}}\left(\mathbf{V}^{\prime}\right)
$$

the resulting $\mathbb{P}^{l}$-equivariant surjective maps (with $\mathbb{U}^{l}$ acting trivially on the target). (These projections should not be confused with the projections $\stackrel{\leftrightarrow}{p}_{\mathbf{V}}$ defined in Sect. 4.1.) Let

$$
p_{l}=\left.\left(\vec{p}^{l}, \overleftarrow{p}^{l}\right)\right|_{\mathfrak{X}^{l}(\tilde{\mathbf{V}})}: \mathfrak{X}^{l}(\tilde{\mathbf{V}}) \rightarrow \mathfrak{X}(\mathbf{V}) \times \mathfrak{X}\left(\mathbf{V}^{\prime}\right)
$$

Again, $p_{l}$ is $\mathbb{P}^{l}$-equivariant (and in particular, $\mathbb{U}^{l}$-invariant) and surjective. 
Define similarly $\stackrel{\leftrightarrow}{\mathbb{E}^{\prime}}(\tilde{\mathbf{V}}), \mathfrak{X}^{\iota^{\prime}}(\tilde{\mathbf{V}}), \mathbb{P}^{\iota^{\prime}}, \mathbb{U}^{\iota^{\prime}}$ and

$$
p_{l^{\prime}}: \mathfrak{X}^{l^{\prime}}(\tilde{\mathbf{V}}) \rightarrow \mathfrak{X}\left(\mathbf{V}^{\prime}\right) \times \mathfrak{X}(\mathbf{V})
$$

by interchanging the roles of $\mathbf{V}$ and $\mathbf{V}^{\prime}$. The parabolic subgroups $\mathbb{P}^{l}$ and $\mathbb{P}^{\prime}$ are opposite with

$\mathbb{M}:=\mathbb{P}^{l} \cap \mathbb{P}^{l^{\prime}}=\left\{g \in \mathbb{G}(\tilde{\mathbf{V}}): g(\mathbf{V})=\mathbf{V}\right.$ and $\left.g\left(\mathbf{V}^{\prime}\right)=\mathbf{V}^{\prime}\right\} \simeq \mathbb{G}(\mathbf{V}) \times \mathbb{G}\left(\mathbf{V}^{\prime}\right)$.

Finally, let

$$
\mathcal{D}_{l, l^{\prime}}: \mathfrak{X}(\mathbf{V}) \times \mathfrak{X}\left(\mathbf{V}^{\prime}\right) \rightarrow \mathfrak{X}(\tilde{\mathbf{V}})
$$

be the diagonal embedding. Its image is

$$
\begin{aligned}
& \mathfrak{X}^{l}(\tilde{\mathbf{V}}) \cap \mathfrak{X}^{\iota^{\prime}}(\tilde{\mathbf{V}}) \\
& \quad=\left\{(A, B) \in \mathfrak{X}(\tilde{\mathbf{V}}): A(\mathbf{V}), B(\mathbf{V}) \subset \mathbf{V} \text { and } A\left(\mathbf{V}^{\prime}\right), B\left(\mathbf{V}^{\prime}\right) \subset \mathbf{V}^{\prime}\right\}
\end{aligned}
$$

and $p_{l} \circ \mathcal{D}_{l, l^{\prime}}=\mathrm{id}$.

\subsection{Sum of multisegments}

Now let $\mathfrak{m}, \mathfrak{m}^{\prime}$ be two multisegments such that $\operatorname{supp} \mathfrak{m}=\operatorname{grdim} \mathbf{V}$ and $\operatorname{supp} \mathfrak{m}^{\prime}=\operatorname{grdim} \mathbf{V}^{\prime}$, and let $\mathfrak{n}=\mathfrak{m}+\mathfrak{m}^{\prime}$, so that $\operatorname{supp} \mathfrak{n}=\operatorname{grdim} \tilde{\mathbf{V}}$. As in Sect. 4.1, let $\mathcal{O}_{\mathfrak{m}}\left(\right.$ resp., $\left.\mathcal{O}_{\mathfrak{m}^{\prime}}, \mathcal{O}_{\mathfrak{n}}\right)$ be the $\mathbb{G}(\mathbf{V})$ (resp., $\mathbb{G}\left(\mathbf{V}^{\prime}\right), \mathbb{G}(\tilde{\mathbf{V}})$ ) orbit in $\overrightarrow{\mathbb{E}}(\mathbf{V})$ (resp., $\overrightarrow{\mathbb{E}}\left(\mathbf{V}^{\prime}\right), \overrightarrow{\mathbb{E}}(\tilde{\mathbf{V}})$ ) corresponding to $\mathfrak{m}$ (resp., $\left.\mathfrak{m}^{\prime}, \mathfrak{n}\right)$. Recall that $\mathfrak{C}_{\mathfrak{m}}^{\circ}=\vec{p}_{\mathbf{V}}^{-1}\left(\mathcal{O}_{\mathfrak{m}}\right), \mathfrak{C}_{\mathfrak{m}^{\prime}}^{\circ}=\vec{p}_{\mathbf{V}^{\prime}}^{-1}\left(\mathcal{O}_{\mathfrak{m}^{\prime}}\right), \mathfrak{C}_{\mathfrak{n}}^{\circ}=\vec{p}_{\tilde{\mathbf{V}}}^{-1}\left(\mathcal{O}_{\mathfrak{n}}\right)$ and $\mathfrak{C}_{\mathfrak{m}}=\overline{\mathfrak{C}_{\mathfrak{m}}^{\circ}}$, $\mathfrak{C}_{\mathfrak{m}^{\prime}}=\overline{\mathfrak{C}_{\mathfrak{m}^{\prime}}^{\circ}}, \mathfrak{C}_{\mathfrak{n}}=\overline{\mathfrak{C}_{\mathfrak{n}}^{\circ}}$ are the respective (Zariski) closures.

Let $\mathfrak{Y}_{\mathfrak{m}, \mathfrak{m}^{\prime}}^{l}$ be the $\mathbb{P}^{l}$-invariant subvariety

$$
\mathfrak{Y}_{\mathfrak{m}, \mathfrak{m}^{\prime}}^{l}=\overline{\mathfrak{C}_{\mathfrak{n}} \cap p_{l}^{-1}\left(\mathfrak{C}_{\mathfrak{m}}^{\circ} \times \mathfrak{C}_{\mathfrak{m}^{\prime}}^{\circ}\right)} \subset \mathfrak{C}_{\mathfrak{n}} \cap p_{l}^{-1}\left(\mathfrak{C}_{\mathfrak{m}} \times \mathfrak{C}_{\mathfrak{m}^{\prime}}\right) .
$$

(We do not know whether the above inclusion can be strict.) Similarly, let

$$
\mathfrak{Y}_{\mathfrak{m}^{\prime}, \mathfrak{m}}^{l^{\prime}}=\overline{\mathfrak{C}_{\mathfrak{n}} \cap p_{l^{\prime}}^{-1}\left(\mathfrak{C}_{\mathfrak{m}^{\prime}}^{\circ} \times \mathfrak{C}_{\mathfrak{m}}^{\circ}\right)} \subset \mathfrak{C}_{\mathfrak{n}} \cap p_{l^{\prime}}^{-1}\left(\mathfrak{C}_{\mathfrak{m}^{\prime}} \times \mathfrak{C}_{\mathfrak{m}}\right) .
$$

Let

$$
\mathfrak{Z}_{\mathfrak{m}, \mathfrak{m}^{\prime}}=\mathfrak{Y}_{\mathfrak{m}, \mathfrak{m}^{\prime}}^{l} \cap \mathfrak{Y}_{\mathfrak{m}^{\prime}, \mathfrak{m}}^{\prime^{\prime}}
$$


Note that

$$
\mathfrak{Z}_{\mathfrak{m}, \mathfrak{m}^{\prime}}=\mathcal{D}_{l, l^{\prime}}\left(\mathfrak{C}_{\mathfrak{m}} \times \mathfrak{C}_{\mathfrak{m}^{\prime}}\right)
$$

since $\supset$ clearly holds while

$$
\mathfrak{Z}_{\mathfrak{m}, \mathfrak{m}^{\prime}} \subset p_{l}^{-1}\left(\mathfrak{C}_{\mathfrak{m}} \times \mathfrak{C}_{\mathfrak{m}^{\prime}}\right) \cap p_{l^{\prime}}^{-1}\left(\mathfrak{C}_{\mathfrak{m}^{\prime}} \times \mathfrak{C}_{\mathfrak{m}}\right)=\mathcal{D}_{l, l^{\prime}}\left(\mathfrak{C}_{\mathfrak{m}} \times \mathfrak{C}_{\mathfrak{m}^{\prime}}\right)
$$

Since $\mathfrak{Y}_{\mathfrak{m}^{\prime}, \mathfrak{m}}^{\iota^{\prime}}$ is $\mathbb{P}^{\iota^{\prime}}$-invariant and $\mathbb{U}^{l} \mathbb{P}^{\iota^{\prime}}$ is dense in $\mathbb{G}(\tilde{\mathbf{V}})$, we have $\overline{\mathbb{G}(\tilde{\mathbf{V}}) \cdot \mathfrak{Y}_{\mathfrak{m}^{\prime}, \mathfrak{m}}^{l^{\prime}}}=\overline{\mathbb{U}^{\imath} \cdot \mathfrak{Y}_{\mathfrak{m}^{\prime}, \mathfrak{m}}^{l^{\prime}}}$.

Also, $\mathbb{P}^{l} \cdot \mathfrak{Z}_{\mathfrak{m}, \mathfrak{m}^{\prime}}=\mathbb{U}^{l} \cdot \mathfrak{Z}_{\mathfrak{m}, \mathfrak{m}^{\prime}}$ since $\mathfrak{Z}_{\mathfrak{m}, \mathfrak{m}^{\prime}}$ is $\mathbb{M}$-invariant.

We can now formulate the main geometric conditions.

Proposition 2 The following conditions are equivalent.

$$
\begin{aligned}
& \left.\mathbb{G}(\tilde{\mathbf{V}}) \cdot \mathfrak{Y}_{\mathfrak{m}^{\prime}, \mathfrak{m}}^{l^{\prime}} \text { (or equivalently, } \mathbb{U}^{l} \cdot \mathfrak{Y}_{\mathfrak{m}^{\prime}, \mathfrak{m}}^{l^{\prime}}\right) \text { is dense in } \mathfrak{C}_{\mathfrak{n}} . \\
& \mathbb{P}^{l} \cdot \mathfrak{Z}_{\mathfrak{m}, \mathfrak{m}^{\prime}} \text { (or equivalently, } \mathbb{U}^{l} \cdot \mathfrak{Z}_{\mathfrak{m}, \mathfrak{m}^{\prime}} \text { is dense in } \mathfrak{Y}_{\mathfrak{m}, \mathfrak{m}^{\prime}}^{l} .
\end{aligned}
$$

Definition 5 We denote the above equivalent conditions by $\operatorname{SG}\left(\mathfrak{m}, \mathfrak{m}^{\prime}\right)$. (This stands for "subrepresentation+geometric") We denote by IG $\left(\mathfrak{m}, \mathfrak{m}^{\prime}\right)$ (for "irreducible+geometric") the condition

$$
\mathbb{G}(\tilde{\mathbf{V}}) \cdot \mathfrak{Z}_{\mathfrak{m}, \mathfrak{m}^{\prime}} \text { is dense in } \mathfrak{C}_{\mathfrak{n}}
$$

Example 1 Let $\mathfrak{m}=\{\rho\}, \mathfrak{m}^{\prime}=\{\vec{\rho}\}$ for some $\rho \in \operatorname{Irr}_{c}$. Note that $\mathfrak{X}(\mathbf{V})=$ $\mathfrak{X}\left(\mathbf{V}^{\prime}\right)=\{(0,0)\}, \mathbb{G}(\tilde{\mathbf{V}})=\mathbb{P}^{l}=\mathbb{P}^{\prime}$ is a two-dimension torus, $\mathbb{U}^{l}=\mathbb{U}^{\iota^{\prime}}=1$, $\overleftrightarrow{\mathbb{E}}(\tilde{\mathbf{V}})$ are one-dimensional and $\mathfrak{X}(\tilde{\mathbf{V}})$ is the "cross" $\mathfrak{C}_{\mathfrak{n}} \cup \mathfrak{C}_{\Delta}$ where $\mathfrak{C}_{\mathfrak{n}}=$ $\{0\} \times \overleftarrow{\mathbb{E}}(\tilde{\mathbf{V}}), \Delta=\{\rho, \vec{\rho}\}, \mathfrak{C}_{\Delta}=\overrightarrow{\mathbb{E}}(\tilde{\mathbf{V}}) \times\{0\}$. Thus, $\mathfrak{Y}_{\mathfrak{m}^{\prime}, \mathfrak{m}}^{\prime^{\prime}}=\mathfrak{Z}_{\mathfrak{m}, \mathfrak{m}^{\prime}}=\{(0,0)\}$ $\mathfrak{Y}_{\mathfrak{m}, \mathfrak{m}^{\prime}}^{l}=\mathfrak{C}_{\mathfrak{n}}$. Hence, $\mathrm{SG}\left(\mathfrak{m}, \mathfrak{m}^{\prime}\right)$ is not satisfied and $\mathrm{SG}\left(\mathfrak{m}^{\prime}, \mathfrak{m}\right)$ is satisfied.

We will give some more equivalent conditions for $\mathrm{SG}\left(\mathfrak{m}, \mathfrak{m}^{\prime}\right)$ in Sect. 5.4 below and ultimately prove Proposition 2 in Sect. 5.8. In the meantime, we state a simple corollary of Proposition 2.

Corollary $2 \mathrm{IG}\left(\mathfrak{m}, \mathfrak{m}^{\prime}\right)$ is the conjunction of $\operatorname{SG}\left(\mathfrak{m}, \mathfrak{m}^{\prime}\right)$ and $\operatorname{SG}\left(\mathfrak{m}^{\prime}, \mathfrak{m}\right)$.

Proof Clearly, IG $\left(\mathfrak{m}, \mathfrak{m}^{\prime}\right)$ implies (16a) and its symmetric analog (interchanging $\mathfrak{m}$ and $\mathfrak{m}^{\prime}$ ). Conversely, (16b) and the symmetric counterpart of (16a) imply that

$$
\overline{\mathbb{G}(\tilde{\mathbf{V}}) \cdot \mathfrak{Z}_{\mathfrak{m}, \mathfrak{m}^{\prime}}}=\overline{\mathbb{G}(\tilde{\mathbf{V}}) \mathbb{P}^{l} \cdot \mathfrak{Z}_{\mathfrak{m}, \mathfrak{m}^{\prime}}}=\overline{\mathbb{G}(\tilde{\mathbf{V}}) \cdot \mathfrak{Y}_{\mathfrak{m}, \mathfrak{m}^{\prime}}^{l}}=\mathfrak{C}_{\mathfrak{n}}
$$

The corollary follows. 


\subsection{The main conjecture}

We now state the new conjecture.

Conjecture 4 Suppose (ALO) of Sect. 3.3. Then,

1. The conditions $\operatorname{SA}\left(\mathfrak{m}, \mathfrak{m}^{\prime}\right)$ and $\operatorname{SG}\left(\mathfrak{m}, \mathfrak{m}^{\prime}\right)$ are equivalent.

2. $Z(\mathfrak{m}) \times Z\left(\mathfrak{m}^{\prime}\right)$ is irreducible if and only if $\operatorname{IG}\left(\mathfrak{m}, \mathfrak{m}^{\prime}\right)$.

Note that the second part of the conjecture would follow from the first one by Corollaries 1 and 2 .

More generally, it would be desirable to have a geometric/combinatorial grasp on $\operatorname{soc}\left(Z(\mathfrak{m}) \times Z\left(\mathfrak{m}^{\prime}\right)\right)$ under $(\mathrm{ALO})$. We hope to get back to this question in the future.

A more ambitious formulation of Conjecture 4 is the following.

Question 1 Are the conditions $\mathrm{SA}\left(\mathfrak{m}, \mathfrak{m}^{\prime}\right)$ and $\operatorname{SG}\left(\mathfrak{m}, \mathfrak{m}^{\prime}\right)$ equivalent even without assuming (ALO) ?

An affirmative answer would give that

$$
\mathrm{IG}\left(\mathfrak{m}, \mathfrak{m}^{\prime}\right) \Longleftrightarrow Z\left(\mathfrak{m}+\mathfrak{m}^{\prime}\right) \text { is a direct summand of } Z(\mathfrak{m}) \times Z\left(\mathfrak{m}^{\prime}\right)
$$

However, it would not directly give an exact irreducibility criterion for $\pi_{1} \times \pi_{2}$ when neither $\pi_{1}$ nor $\pi_{2}$ is $\square$-irreducible.

Remark 2 1. As we shall see in Sect. 5.4 below, the condition $G L S(\mathfrak{m})$ implies $\operatorname{SG}(\mathfrak{m}, \mathfrak{m})$ for any $\mathfrak{m}$. (This is consistent with Conjecture 3 and the fact that $\operatorname{SSA}(\mathfrak{m}, \mathfrak{m})$ implies $\operatorname{SA}(\mathfrak{m}, \mathfrak{m})$.)

2. By inspection, for $\mathfrak{m}$ consisting of up to 6 segments, $S G(\mathfrak{m}, \mathfrak{m})$ is equivalent to the multiplicity freeness of $Z(\mathfrak{m}) \times Z(\mathfrak{m})$, and in particular it implies $\mathrm{SA}(\mathfrak{m}, \mathfrak{m})$ in these cases (cf. Remark 1). Leclerc's example (12) shows that in general, the condition $\operatorname{SG}(\mathfrak{m}, \mathfrak{m})$ is strictly weaker than $G L S(\mathfrak{m})$.

3. If neither $G L S(\mathfrak{m})$ nor $G L S\left(\mathfrak{m}^{\prime}\right)$ is satisfied, then at the moment we do not have a conjectural geometric criterion for either $\operatorname{SSA}\left(\mathfrak{m}, \mathfrak{m}^{\prime}\right)$ or the irreducibility of $Z(\mathfrak{m}) \times Z\left(\mathfrak{m}^{\prime}\right)(\mathrm{cf} .(11 b))$.

\subsection{Linearization}

Before proving the equivalence of the conditions in Proposition 2 we will first linearize and explicate them in a way similar to what was done for the condition $G L S(\mathfrak{m})$ in Sect. 4.3.

Write $\mathfrak{m}=\sum_{i \in I} \Delta_{i}$ and $\mathfrak{m}^{\prime}=\sum_{i^{\prime} \in I^{\prime}} \Delta_{i^{\prime}}$ with $I \cap I^{\prime}=\emptyset$. Fix $\mathbf{A}_{\mathfrak{m}} \in \mathcal{O}_{\mathfrak{m}}$ and $\mathbf{A}_{\mathfrak{m}^{\prime}} \in \mathcal{O}_{\mathfrak{m}^{\prime}}$ and let $\mathbf{A}_{\mathfrak{n}}=\mathbf{A}_{\mathfrak{m}} \oplus \mathbf{A}_{\mathfrak{m}^{\prime}} \in \mathcal{O}_{\mathfrak{n}}$. We take a graded basis $x_{\rho, i}$, $\rho \in \Delta_{i}, i \in I$ (resp., $i \in I^{\prime}$ ) for $\mathbf{V}$ (resp. $\mathbf{V}^{\prime}$ ) for which $\mathbf{A}_{\mathfrak{m}}$ (resp., $\mathbf{A}_{\mathfrak{m}^{\prime}}$ ) has a 
graded Jordan normal form (14). Thus, $\mathbf{A}_{\mathfrak{n}}$ has a graded Jordan normal form with respect to the union $x_{\rho, i}, \rho \in \Delta_{i}, i \in I \cup I^{\prime}$. Let

$$
X_{\mathfrak{m}, \mathfrak{m}^{\prime}}=\left\{\left(i, i^{\prime}\right) \in I \times I^{\prime}: \Delta_{i} \prec \Delta_{i^{\prime}}\right\}
$$

and

$$
Y_{\mathfrak{m}, \mathfrak{m}^{\prime}}=\left\{\left(i, i^{\prime}\right) \in I \times I^{\prime}: \Delta_{i} \prec \vec{\Delta}_{i^{\prime}}\right\}
$$

so that $X_{\mathfrak{m}}=X_{\mathfrak{m}, \mathfrak{m}}$ and $Y_{\mathfrak{m}}=Y_{\mathfrak{m}, \mathfrak{m}}$ (see Sect. 4.3). We have $X_{\mathfrak{n}}=X_{\mathfrak{m}} \cup$ $X_{\mathfrak{m}^{\prime}} \cup X_{\mathfrak{m}, \mathfrak{m}^{\prime}} \cup X_{\mathfrak{m}^{\prime}, \mathfrak{m}}$ and $Y_{\mathfrak{n}}=Y_{\mathfrak{m}} \cup Y_{\mathfrak{m}^{\prime}} \cup Y_{\mathfrak{m}, \mathfrak{m}^{\prime}} \cup Y_{\mathfrak{m}^{\prime}, \mathfrak{m}}$ (disjoint unions). As in Sect. 4.3, let $\mathbf{C}_{\mathfrak{m}}, \mathbf{C}_{\mathfrak{m}^{\prime}}$ and $\mathbf{C}_{\mathfrak{n}}$ be the centralizers of $\mathbf{A}_{\mathfrak{m}} \mathbf{A}_{\mathfrak{m}^{\prime}}$ and $\mathbf{A}_{\mathfrak{n}}$ in $\overleftarrow{\mathbb{E}}(\mathbf{V}), \overleftarrow{\mathbb{E}}\left(\mathbf{V}^{\prime}\right)$ and $\overleftarrow{\mathbb{E}}(\tilde{\mathbf{V}})$ respectively. Similarly, let $\mathbf{G}_{\mathfrak{m}}$ (resp., $\left.\mathbf{G}_{\mathfrak{m}^{\prime}}, \mathbf{G}_{\mathfrak{n}}\right)$ be the centralizer of $\mathbf{A}_{\mathfrak{m}}\left(\right.$ resp., $\left.\mathbf{A}_{\mathfrak{m}^{\prime}}, \mathbf{A}_{\mathfrak{n}}\right)$ in $\mathbb{G}(\mathbf{V})$ (resp., $\mathbb{G}\left(\mathbf{V}^{\prime}\right), \mathbb{G}(\tilde{\mathbf{V}})$ ). Let $\mathbf{C}_{\mathfrak{n}}^{l}=\mathbf{C}_{\mathfrak{n}} \cap \overleftarrow{\mathbb{E}^{l}}(\tilde{\mathbf{V}})$. Thus, $\mathbf{C}_{\mathfrak{n}}^{l}$ consists of the elements of $\mathbf{C}_{\mathfrak{n}}$ whose $X_{\mathfrak{m}^{\prime}, \mathfrak{m}^{-}}$ coordinates vanish. Define similarly $\mathbf{C}_{\mathfrak{n}}^{l^{\prime}}$ and let $\mathbf{C}_{\mathfrak{n}}^{l, l^{\prime}}=\mathbf{C}_{\mathfrak{n}}^{l} \cap \mathbf{C}_{\mathfrak{n}}^{l^{\prime}} \simeq \mathbf{C}_{\mathfrak{m}} \oplus \mathbf{C}_{\mathfrak{m}^{\prime}}$. Let

$$
\begin{aligned}
\mathbf{U}^{l} & =\mathbf{G}_{\mathfrak{n}} \cap \mathbb{U}^{l}=\left\{g \in \mathbf{G}_{\mathfrak{n}}: g_{i, j}=\delta_{i, j} \text { for all }(i, j) \in Y_{\mathfrak{n}} \backslash Y_{\mathfrak{m}, \mathfrak{m}^{\prime}}\right\}, \\
\mathbf{P}^{l} & =\mathbf{G}_{\mathfrak{n}} \cap \mathbb{P}^{l}=\left\{g \in \mathbf{G}_{\mathfrak{n}}: g_{i, j}=0 \text { for all }(i, j) \in Y_{\mathfrak{m}^{\prime}, \mathfrak{m}}\right\}, \\
\mathbf{U}^{\iota^{\prime}} & =\mathbf{G}_{\mathfrak{n}} \cap \mathbb{U}^{\iota^{\prime}}=\left\{g \in \mathbf{G}_{\mathfrak{n}}: g_{i, j}=\delta_{i, j} \text { for all }(i, j) \in Y_{\mathfrak{n}} \backslash Y_{\mathfrak{m}^{\prime}, \mathfrak{m}}\right\}, \\
\mathbf{P}^{\iota^{\prime}} & =\mathbf{G}_{\mathfrak{n}} \cap \mathbb{P}^{\iota^{\prime}}=\left\{g \in \mathbf{G}_{\mathfrak{n}}: g_{i, j}=0 \text { for all }(i, j) \in Y_{\mathfrak{m}, \mathfrak{m}^{\prime}}\right\}, \\
\mathbf{M} & =\mathbf{G}_{\mathfrak{n}} \cap \mathbb{M}=\mathbf{P}^{l} \cap \mathbf{P}^{\iota^{\prime}} \simeq \mathbf{G}_{\mathfrak{m}} \times \mathbf{G}_{\mathfrak{m}^{\prime}},
\end{aligned}
$$

so that

$$
\mathbf{P}^{l}=\mathbf{M} \ltimes \mathbf{U}^{l}, \quad \mathbf{P}^{i^{\prime}}=\mathbf{M} \ltimes \mathbf{U}^{l^{\prime}} .
$$

The Lie algebra of $\mathbf{U}^{l}$ is spanned by $\beta_{i, j}^{\mathfrak{n}},(i, j) \in Y_{\mathfrak{m}, \mathfrak{m}^{\prime}}$.

Proposition 3 We have $\overline{\mathbf{G}_{\mathfrak{n}} \cdot \mathbf{C}_{\mathfrak{n}}^{l^{\prime}}}=\overline{\mathbf{U}^{l} \cdot \mathbf{C}_{\mathfrak{n}}^{\iota^{\prime}}}$ and $\mathbf{P}^{l} \cdot \mathbf{C}_{\mathfrak{n}}^{l, l^{\prime}}=\mathbf{U}^{l} \cdot \mathbf{C}_{\mathfrak{n}}^{l, l^{\prime}}$. Moreover, the following conditions are equivalent.

1. $\mathbf{G}_{\mathfrak{n}} \cdot \mathbf{C}_{\mathfrak{n}}^{l^{\prime}}$ (or equivalently, $\mathbf{U}^{l} \cdot \mathbf{C}_{\mathfrak{n}}^{l^{\prime}}$ ) is dense in $\mathbf{C}_{\mathfrak{n}}$.

2. $\mathbf{P}^{l} \cdot \mathbf{C}_{\mathfrak{n}}^{l, l^{\prime}}$ (or equivalently. $\mathbf{U}^{l} \cdot \mathbf{C}_{\mathfrak{n}}^{l, l^{\prime}}$ ) is dense in $\mathbf{C}_{\mathfrak{n}}^{l}$.

3. There exist $\lambda \in \mathbf{C}_{\mathfrak{m}}$ and $\lambda^{\prime} \in \mathbf{C}_{\mathfrak{m}^{\prime}}$ with coordinates $\lambda_{i, j},(i, j) \in X_{\mathfrak{m}}$ and $\lambda_{i, j}^{\prime},(i, j) \in X_{\mathfrak{m}^{\prime}}$ respectively, such that the vectors $v_{i, j}^{\mathfrak{m}, \mathfrak{m}^{\prime}}\left(\lambda, \lambda^{\prime}\right),(i, j) \in$ $X_{\mathfrak{m}, \mathfrak{m}^{\prime}}$ given by 


$$
\begin{aligned}
v_{i, j}^{\mathfrak{m}, \mathfrak{m}^{\prime}}\left(\lambda, \lambda^{\prime}\right)= & \sum_{r \in I:(i, r) \in X_{\mathfrak{m}},(r, j) \in Y_{\mathfrak{m}, \mathfrak{m}^{\prime}}} \lambda_{i, r} y_{r, j} \\
& -\sum_{s \in I^{\prime}:(s, j) \in X_{\mathfrak{m}^{\prime}},(i, s) \in Y_{\mathfrak{m}, \mathfrak{m}^{\prime}}} \lambda_{s, j}^{\prime} y_{i, s}
\end{aligned}
$$

are linearly independent in the complex vector space $\mathbb{C}^{Y_{\mathfrak{m}, \mathfrak{m}^{\prime}} \text { with standard }}$ basis $\left\{y_{i, j}:(i, j) \in Y_{\mathfrak{m}, \mathfrak{m}^{\prime}}\right\}$.

Finally, $\mathrm{SG}\left(\mathfrak{m}, \mathfrak{m}^{\prime}\right)$ is equivalent to the conditions above.

In the rest of the section we will prove Proposition 3.

Remark 3 Assume that Proposition 3 holds. Then,

1. By the same argument as in Corollary 2 (or alternatively, using Remark 4 below), $\operatorname{IG}\left(\mathfrak{m}, \mathfrak{m}^{\prime}\right)$ is equivalent to the condition

$$
\mathbf{G}_{\mathfrak{n}} \cdot \mathbf{C}_{\mathfrak{n}}^{l, l^{\prime}} \text { is dense in } \mathbf{C}_{\mathfrak{n}} .
$$

2. For any $\mathfrak{m}, G L S(\mathfrak{m})$ implies $S G(\mathfrak{m}, \mathfrak{m})$, since in the notation of Lemma 1 we have

$$
v_{i, j}^{\mathfrak{m}}(\lambda)=v_{i, j}^{\mathfrak{m}, \mathfrak{m}}(\lambda, \lambda)
$$

for $(i, j) \in X_{\mathfrak{m}}$ and $\lambda \in \mathbf{C}_{\mathfrak{m}}$.

3. The linear independence of (17) is an open and $\mathbf{G}_{\mathfrak{m}} \times \mathbf{G}_{\mathfrak{m}^{\prime}}$-invariant condition in $\left(\lambda, \lambda^{\prime}\right) \in \mathbf{C}_{\mathfrak{m}} \times \mathbf{C}_{\mathfrak{m}^{\prime}}$.

4. The conditions $\operatorname{SG}\left(\mathfrak{m}, \mathfrak{m}^{\prime}\right)$ and $\mathrm{SG}\left(\mathfrak{m}^{\prime \vee}, \mathfrak{m}^{\vee}\right)$ are equivalent, since $X_{\mathfrak{m}, \mathfrak{m}^{\prime}}=$ $X_{\mathfrak{m}^{\prime}, \mathfrak{m}^{\vee}}$ and $Y_{\mathfrak{m}, \mathfrak{m}^{\prime}}=Y_{\mathfrak{m}^{\vee}, \mathfrak{m}^{\vee}}$. Thus, taking into account (9), in Conjecture 4 we may assume without loss of generality that $Z(\mathfrak{m})$ is $\square$-irreducible.

5. Condition 3 is the least conceptual but the most practical to check. It can be easily implemented on a computer, at least as a randomized algorithm. Nonetheless, it would be interesting to replace it by a deterministic criterion.

\subsection{Proof of first part of Proposition 3}

Since $\mathbf{C}_{\mathfrak{n}}^{l, l^{\prime}}$ is $\mathbf{M}$-invariant and $\mathbf{P}^{l}=\mathbf{M} \ltimes \mathbf{U}^{l}$, it is clear that $\mathbf{P}^{l} \cdot \mathbf{C}_{\mathfrak{n}}^{l, l^{\prime}}=\mathbf{U}^{l} \cdot \mathbf{C}_{\mathfrak{n}}^{l, l^{\prime}}$. Also, since $\mathbf{U}^{l} \mathbf{P}^{t^{\prime}}$ is dense in $\mathbf{G}_{\mathfrak{n}}$ (e.g., by considering the Lie algebras) and $\mathbf{C}_{\mathfrak{n}}^{l^{\prime}}$ is $\mathbf{P}^{l^{\prime}}$-invariant, we have $\overline{\mathbf{G}_{\mathfrak{n}} \cdot \mathbf{C}_{\mathfrak{n}}^{\iota^{\prime}}}=\overline{\mathbf{U}^{\imath} \cdot \mathbf{C}_{\mathfrak{n}}^{\iota^{\prime}}}$.

Next, we show the equivalence of conditions 1, 2 and 3 of Proposition 3. We use the following simple general criterion. 
Remark 4 Suppose that $G$ is a linear algebraic group with a rational (linear) representation on a finite-dimensional vector space $W$ and let $W^{\prime}$ be a subspace of $W$. Then,

$$
G \cdot W^{\prime} \text { is dense in } W \Longleftrightarrow \exists w \in W^{\prime} \text { such that } \operatorname{Lie}(G) \cdot w+W^{\prime}=W .
$$

Returning to the case at hand, we identify

$$
\mathbf{C}_{\mathfrak{n}}^{l} / \mathbf{C}_{\mathfrak{n}}^{l, l^{\prime}} \simeq \mathbf{C}_{\mathfrak{n}} / \mathbf{C}_{\mathfrak{n}}^{l^{\prime}}
$$

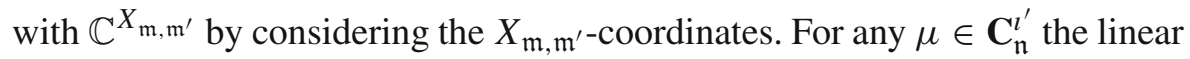
map

$$
u \in \operatorname{Lie}\left(\mathbf{U}^{l}\right) \mapsto u \cdot \mu+\mathbf{C}_{\mathfrak{n}}^{l^{\prime}}
$$

gives rise to a linear map

$$
L_{\mu}: \mathbb{C}^{Y_{\mathfrak{m}, \mathfrak{m}^{\prime}}} \rightarrow \mathbb{C}^{X_{\mathfrak{m}, \mathfrak{m}^{\prime}}}
$$

which depends only on the image $\left(\lambda, \lambda^{\prime}\right)$ of $\mu$ under the projection $\mathbf{C}_{\mathfrak{n}}^{l^{\prime}} \rightarrow$ $\mathbf{C}_{\mathfrak{m}} \oplus \mathbf{C}_{\mathfrak{m}^{\prime}}$. Thus, by Remark 4, the conditions 1 and 2 of Proposition 3 are both equivalent to the surjectivity of $L_{\mu}$ for some $\mu \in \mathbf{C}_{\mathfrak{n}}^{l, l^{\prime}}$. Condition 3 is merely an explication of this (or more precisely, of the injectivity of the dual map).

\subsection{A simple reduction}

It remains to show the equivalence of each of the conditions in Proposition 2 with the corresponding condition in Proposition 3, thus completing the proof of the two propositions.

We use the following simple result.

Lemma 2 Let $G$ be a linear algebraic group acting algebraically on quasiaffine varieties $X$ and $Y$ and let $p: X \rightarrow Y$ be a $G$-equivariant morphism. Let $H$ be a closed subgroup of $G$ and let $W$ be an $H$-invariant subvariety of $X$. Assume that $G$ acts transitively on $Y$ and fix $y_{0} \in Y$. Let $G_{0}$ be the stabilizer of $y_{0}$ in $G, X_{0}=p^{-1}\left(y_{0}\right)$ and $W_{0}=W \cap X_{0}$. Assume that $X_{0}$ is irreducible and that $W=H \cdot W_{0}$. Then, $G \cdot W=G \cdot W_{0}$ and

$$
G \cdot W \text { is dense in } X \Longleftrightarrow G_{0} \cdot W_{0} \text { is dense in } X_{0} \text {. }
$$

Proof The first assertion is clear since $W=H \cdot W_{0}$. Suppose that $\overline{G_{0} \cdot W_{0}}=$ $X_{0}$. Then $\overline{G \cdot W_{0}}=\overline{G \cdot G_{0} \cdot W_{0}}=\overline{G \cdot X_{0}} \supset G \cdot X_{0}=X$ by the transitivity 
of $G$ on $Y$. Conversely, suppose that $\overline{G \cdot W_{0}}=X$. Then $G \cdot W_{0}$ contains an open dense (and without loss of generality, $G$-invariant) subset $U$ of $X$. The set

$$
\left\{y \in Y: p^{-1}(y) \cap U \neq \emptyset\right\}
$$

is $G$-invariant and non-empty (and in fact dense in $Y$ ). Since $G$ acts transitively on $Y$ we infer that $p^{-1}(y) \cap U \neq \emptyset$ for all $y \in Y$. In particular, $X_{0} \cap U$ is a non-empty open subset of $X_{0}$, hence dense since $X_{0}$ is irreducible. A fortiori, $X_{0} \cap G \cdot W_{0}$ is dense in $X_{0}$. However, $X_{0} \cap G \cdot W_{0}=G_{0} \cdot W_{0}$ since if $g x_{0} \in X_{0}$ where $x_{0} \in X_{0}$ and $g \in G$, then necessarily $g \in G_{0}$. Hence, $G_{0} \cdot W_{0}$ is dense in $X_{0}$ as required.

\subsection{An auxiliary result}

We go back to the setup of Sect. 5.2. In order to invoke Lemma 2 we will need an additional result.

Lemma 3 We have the following equalities of spaces.

$$
\begin{aligned}
& \mathbb{U}^{l} \cdot \mathbf{A}_{\mathfrak{n}}=\overline{\mathcal{O}_{\mathfrak{n}}} \cap\left(\vec{p}^{l}\right)^{-1}\left(\mathbf{A}_{\mathfrak{m}}, \mathbf{A}_{\mathfrak{m}^{\prime}}\right)=\mathcal{O}_{\mathfrak{n}} \cap\left(\vec{p}^{l}\right)^{-1}\left(\mathbf{A}_{\mathfrak{m}}, \mathbf{A}_{\mathfrak{m}^{\prime}}\right), \\
& \mathbb{P}^{l} \cdot \mathbf{A}_{\mathfrak{n}}=\overline{\mathcal{O}_{\mathfrak{n}}} \cap\left(\vec{p}^{l}\right)^{-1}\left(\mathcal{O}_{\mathfrak{m}} \times \mathcal{O}_{\mathfrak{m}^{\prime}}\right)=\mathcal{O}_{\mathfrak{n}} \cap\left(\vec{p}^{l}\right)^{-1}\left(\mathcal{O}_{\mathfrak{m}} \times \mathcal{O}_{\mathfrak{m}^{\prime}}\right), \\
& \mathfrak{C}_{\mathfrak{n}} \cap p_{l}^{-1}\left(\mathfrak{C}_{\mathfrak{m}}^{\circ} \times \mathfrak{C}_{\mathfrak{m}^{\prime}}^{\circ}\right)=\mathfrak{C}_{\mathfrak{n}}^{\circ} \cap p_{l}^{-1}\left(\mathfrak{C}_{\mathfrak{m}}^{\circ} \times \mathfrak{C}_{\mathfrak{m}^{\prime}}^{\circ}\right)=\mathbb{P}^{l} \cdot\left(\vec{p}_{\tilde{\mathbf{V}}}^{-1}\left(\mathbf{A}_{\mathfrak{n}}\right) \cap \mathfrak{X}^{l}(\tilde{\mathbf{V}})\right) .
\end{aligned}
$$

A similar statement holds for $\iota^{\prime}$.

Proof By the $\mathbb{P}^{l}$-equivariant of the map $\vec{p}^{l}$ and the surjectivity of the map $\mathbb{P}^{\imath} \rightarrow \mathbb{G}(\mathbf{V}) \times \mathbb{G}\left(\mathbf{V}^{\prime}\right)$, we have

$$
\begin{aligned}
\mathbb{P}^{l} \cdot\left(\left(\vec{p}^{l}\right)^{-1}\left(\mathbf{A}_{\mathfrak{m}}, \mathbf{A}_{\mathfrak{m}^{\prime}}\right)\right) & =\left(\vec{p}^{l}\right)^{-1}\left(\mathbb{G}(\mathbf{V}) \cdot \mathbf{A}_{\mathfrak{m}}, \mathbb{G}\left(\mathbf{V}^{\prime}\right) \cdot \mathbf{A}_{\mathfrak{m}^{\prime}}\right) \\
& =\left(\vec{p}^{l}\right)^{-1}\left(\mathcal{O}_{\mathfrak{m}} \times \mathcal{O}_{\mathfrak{m}^{\prime}}\right)
\end{aligned}
$$

Hence, the equality (18a) implies (18b)

Next, we show that (18b) implies (18c). Indeed, assuming (18b), we have

$$
\begin{aligned}
& \mathbb{P}^{l} \cdot\left(\vec{p}_{\tilde{\mathbf{V}}}^{-1}\left(\mathbf{A}_{\mathfrak{n}}\right)\right)=\vec{p}_{\tilde{\mathbf{V}}}^{-1}\left(\mathbb{P}^{l} \cdot \mathbf{A}_{\mathfrak{n}}\right)=\vec{p}_{\tilde{\mathbf{V}}}^{-1}\left(\mathcal{O}_{\mathfrak{n}} \cap\left(\vec{p}^{l}\right)^{-1}\left(\mathcal{O}_{\mathfrak{m}} \times \mathcal{O}_{\mathfrak{m}^{\prime}}\right)\right) \\
= & \mathfrak{C}_{\mathfrak{n}}^{\circ} \cap\left(\vec{p}^{l} \circ \vec{p}_{\tilde{\mathbf{V}}}\right)^{-1}\left(\mathcal{O}_{\mathfrak{m}} \times \mathcal{O}_{\mathfrak{m}^{\prime}}\right) .
\end{aligned}
$$


Since

$$
\left(\vec{p}_{\mathbf{V}}, \vec{p}_{\mathbf{V}^{\prime}}\right) \circ p_{l}=\left.\vec{p}^{l} \circ \vec{p}_{\tilde{\mathbf{V}}}\right|_{\mathfrak{X}^{l}(\tilde{\mathbf{V}})},
$$

we deduce that

$$
\begin{aligned}
\mathbb{P}^{l} \cdot\left(\vec{p}_{\tilde{\mathbf{V}}}^{-1}\left(\mathbf{A}_{\mathfrak{n}}\right)\right) \cap \mathfrak{X}^{l}(\tilde{\mathbf{V}}) & =\mathfrak{C}_{\mathfrak{n}}^{\circ} \cap p_{l}^{-1}\left(\vec{p}_{\mathbf{V}}^{-1}\left(\mathcal{O}_{\mathfrak{m}}\right) \times \vec{p}_{\mathbf{V}^{\prime}}^{-1}\left(\mathcal{O}_{\mathfrak{m}^{\prime}}\right)\right) \\
& =\mathfrak{C}_{\mathfrak{n}}^{\circ} \cap p_{l}^{-1}\left(\mathfrak{C}_{\mathfrak{m}}^{\circ} \times \mathfrak{C}_{\mathfrak{m}^{\prime}}^{\circ}\right) .
\end{aligned}
$$

Also,

$$
\begin{aligned}
& \vec{p}_{\tilde{\mathbf{V}}}\left(\mathfrak{C}_{\mathfrak{n}} \cap p_{l}^{-1}\left(\mathfrak{C}_{\mathfrak{m}}^{\circ} \times \mathfrak{C}_{\mathfrak{m}^{\prime}}^{\circ}\right)\right) \subset \vec{p}_{\tilde{\mathbf{V}}}\left(\mathfrak{C}_{\mathfrak{n}}\right) \cap \vec{p}_{\tilde{\mathbf{V}}}\left(p_{l}^{-1}\left(\mathfrak{C}_{\mathfrak{m}}^{\circ} \times \mathfrak{C}_{\mathfrak{m}^{\prime}}^{\circ}\right)\right) \subset \\
& \overline{\mathcal{O}_{\mathfrak{n}}} \cap\left(\vec{p}^{l}\right)^{-1}\left(\vec{p}_{\mathbf{V}}\left(\mathfrak{C}_{\mathfrak{m}}^{\circ}\right), \vec{p}_{\mathbf{V}^{\prime}}\left(\mathfrak{C}_{\mathfrak{m}^{\prime}}^{\circ}\right)\right)=\overline{\mathcal{O}_{\mathfrak{n}}} \cap\left(\vec{p}^{l}\right)^{-1}\left(\mathcal{O}_{\mathfrak{m}} \times \mathcal{O}_{\mathfrak{m}^{\prime}}\right) \subset \mathcal{O}_{\mathfrak{n}}
\end{aligned}
$$

by (18b) and hence

$$
\mathfrak{C}_{\mathfrak{n}} \cap p_{l}^{-1}\left(\mathfrak{C}_{\mathfrak{m}}^{\circ} \times \mathfrak{C}_{\mathfrak{m}^{\prime}}^{\circ}\right) \subset \vec{p}_{\tilde{\mathbf{v}}}^{-1}\left(\mathcal{O}_{\mathfrak{n}}\right)=\mathfrak{C}_{\mathfrak{n}}^{\circ}
$$

as required.

Note that the statements with respect to $\imath^{\prime}$ are obtained from the original ones by interchanging $\mathfrak{m}$ and $\mathfrak{m}^{\prime}$. Thus, it remains to prove (18a).

Since $\vec{p}^{l}$ is $\mathbb{U}^{l}$-invariant, the $\mathbb{U}^{l}$-orbit of $\mathbf{A}_{\mathfrak{n}}$ is contained in $\mathcal{O}_{\mathfrak{n}} \cap$ $\left(\vec{p}^{l}\right)^{-1}\left(\mathbf{A}_{\mathfrak{m}}, \mathbf{A}_{\mathfrak{m}^{\prime}}\right)$.

Next, we show that

$$
\overline{\mathcal{O}_{\mathfrak{n}}} \cap\left(\vec{p}^{l}\right)^{-1}\left(\mathbf{A}_{\mathfrak{m}}, \mathbf{A}_{\mathfrak{m}^{\prime}}\right) \subset \mathcal{O}_{\mathfrak{n}}
$$

Indeed, recall that

$$
\mathcal{O}_{\mathfrak{n}}=\left\{D \in \overrightarrow{\mathbb{E}}(\tilde{\mathbf{V}}):\left.\operatorname{rk} D^{r}\right|_{\tilde{\mathbf{V}}_{\rho}}=\left.\operatorname{rk} \mathbf{A}_{\mathfrak{n}}^{r}\right|_{\tilde{\mathbf{V}}_{\rho}} \forall \rho \in \operatorname{Irr}_{c}, r \geq 0\right\}
$$

and

$$
\overline{\mathcal{O}_{\mathfrak{n}}}=\left\{D \in \overrightarrow{\mathbb{E}}(\tilde{\mathbf{V}}):\left.\operatorname{rk} D^{r}\right|_{\tilde{\mathbf{V}}_{\rho}} \leq\left.\operatorname{rk} \mathbf{A}_{\mathfrak{n}}^{r}\right|_{\tilde{\mathbf{V}}_{\rho}} \forall \rho \in \operatorname{Irr}_{c}, r \geq 0\right\} .
$$

On the other hand, for any $D \in\left(\vec{p}^{l}\right)^{-1}\left(\mathbf{A}_{\mathfrak{m}}, \mathbf{A}_{\mathfrak{m}^{\prime}}\right)$ we have

$$
\left.\operatorname{rk} D^{r}\right|_{\tilde{\mathbf{V}}_{\rho}} \geq\left.\operatorname{rk} \mathbf{A}_{\mathfrak{m}}^{r}\right|_{\mathbf{V}_{\rho}}+\left.\operatorname{rk} \mathbf{A}_{\mathfrak{m}^{\prime}}^{r}\right|_{\mathbf{V}_{\rho}^{\prime}}=\left.\operatorname{rk} \mathbf{A}_{\mathfrak{n}}^{r}\right|_{\tilde{\mathbf{V}}_{\rho}}
$$

Our claim follows. 
It remains to show that $\mathcal{O}_{\mathfrak{n}} \cap\left(\vec{p}^{l}\right)^{-1}\left(\mathbf{A}_{\mathfrak{m}}, \mathbf{A}_{\mathfrak{m}^{\prime}}\right)$ is contained in the $\mathbb{U}^{l}$-orbit of $\mathbf{A}_{\mathfrak{n}}$.

Consider the abelian category whose objects are pairs $(U, D)$ where $U$ is a finite-dimensional $\operatorname{Irr}_{c}$-graded vector space and $D \in \overrightarrow{\mathbb{E}}(U)$; the morphisms between $(U, D)$ and $\left(U^{\prime}, D^{\prime}\right)$ are the grading preserving linear transformations $T: U \rightarrow U^{\prime}$ such that $T \circ D=D^{\prime} \circ T$.

The statement that we need to prove is that if

$$
0 \rightarrow\left(\mathbf{V}, \mathbf{A}_{\mathfrak{m}}\right) \stackrel{\iota}{\rightarrow}(\tilde{\mathbf{V}}, D) \stackrel{J}{\rightarrow}\left(\mathbf{V}^{\prime}, \mathbf{A}_{\mathfrak{m}^{\prime}}\right) \rightarrow 0
$$

is a short exact sequence and $(\tilde{\mathbf{V}}, D) \simeq\left(\tilde{\mathbf{V}}, \mathbf{A}_{\mathfrak{n}}\right)=\left(\mathbf{V}, \mathbf{A}_{\mathfrak{m}}\right) \oplus\left(\mathbf{V}^{\prime}, \mathbf{A}_{\mathfrak{m}^{\prime}}\right)$, then (19) splits. In fact, this is true for any locally finite $\mathbb{C}$-linear abelian category. Indeed, we have an exact sequence

$$
\begin{array}{r}
0 \rightarrow \operatorname{Hom}\left(\left(\mathbf{V}^{\prime}, \mathbf{A}_{\mathfrak{m}^{\prime}}\right),\left(\mathbf{V}, \mathbf{A}_{\mathfrak{m}}\right)\right) \stackrel{J_{*}^{*}}{\rightarrow} \operatorname{Hom}\left((\tilde{\mathbf{V}}, D),\left(\mathbf{V}, \mathbf{A}_{\mathfrak{m}}\right)\right) \stackrel{l_{*}}{\rightarrow} \\
\operatorname{Hom}\left(\left(\mathbf{V}, \mathbf{A}_{\mathfrak{m}}\right),\left(\mathbf{V}, \mathbf{A}_{\mathfrak{m}}\right)\right)
\end{array}
$$

and

$$
\begin{aligned}
\operatorname{Hom}\left((\tilde{\mathbf{V}}, D),\left(\mathbf{V}, \mathbf{A}_{\mathfrak{m}}\right)\right) \simeq & \operatorname{Hom}\left(\left(\mathbf{V}^{\prime}, \mathbf{A}_{\mathfrak{m}^{\prime}}\right),\left(\mathbf{V}, \mathbf{A}_{\mathfrak{m}}\right)\right) \\
& \oplus \operatorname{Hom}\left(\left(\mathbf{V}, \mathbf{A}_{\mathfrak{m}}\right),\left(\mathbf{V}, \mathbf{A}_{\mathfrak{m}}\right)\right) .
\end{aligned}
$$

Comparing dimensions we infer that $l_{*}$ is onto. Hence, (19) splits.

\subsection{Conclusion of proof of Propositions 2 and 3}

Using Lemmas 2 and 3 we show the equivalence of (16a) and (16b) with the first (resp., second) condition in Proposition 3, thereby completing the proofs of Propositions 2 and 3.

For the first equivalence we apply Lemma 2 to

$$
W=\mathfrak{C}_{\mathfrak{n}}^{\circ} \cap p_{l^{\prime}}^{-1}\left(\mathfrak{C}_{\mathfrak{m}^{\prime}}^{\circ} \times \mathfrak{C}_{\mathfrak{m}}^{\circ}\right) \hookrightarrow X=\mathfrak{C}_{\mathfrak{n}}^{\circ} \stackrel{\vec{p}_{\tilde{\mathbf{v}}}}{\longrightarrow} Y=\mathcal{O}_{\mathfrak{n}} \ni y_{0}=\mathbf{A}_{\mathfrak{n}},
$$

with $G=\mathbb{G}(\tilde{\mathbf{V}})$ and $H=\mathbb{P}^{t^{\prime}}$. Note that $G_{0}=\mathbf{G}_{\mathfrak{n}}$ and the embedding $W_{0} \hookrightarrow X_{0}$ can be identified via $\overleftarrow{p}_{\tilde{\mathbf{V}}}$ with $\mathbf{C}_{\mathfrak{n}}^{l^{\prime}} \hookrightarrow \mathbf{C}_{\mathfrak{n}}$. By Lemma 3 (with respect to $\iota^{\prime}$ ) we have $W=H \cdot W_{0}$ and $\mathfrak{Y}_{\mathfrak{m}^{\prime}, \mathfrak{m}}^{l^{\prime}}=\bar{W}$. Also, by definition, $\mathfrak{C}_{\mathfrak{n}}=\bar{X}$. 
For the second equivalence, we apply Lemma 2 with

$$
\begin{gathered}
W=\mathcal{D}_{l, l^{\prime}}\left(\mathfrak{C}_{\mathfrak{m}}^{\circ} \times \mathfrak{C}_{\mathfrak{m}^{\prime}}^{\circ}\right) \hookrightarrow X=p_{l}^{-1}\left(\mathfrak{C}_{\mathfrak{m}}^{\circ} \times \mathfrak{C}_{\mathfrak{m}^{\prime}}^{\circ}\right) \cap \mathfrak{C}_{\mathfrak{n}}^{\circ} \stackrel{\vec{p}_{\tilde{\mathbf{v}}}}{\longrightarrow} \\
Y=\mathcal{O}_{\mathfrak{n}} \cap\left(\vec{p}^{l}\right)^{-1}\left(\mathcal{O}_{\mathfrak{m}} \times \mathcal{O}_{\mathfrak{m}^{\prime}}\right) \ni y_{0}=\mathbf{A}_{\mathfrak{n}},
\end{gathered}
$$

$G=\mathbb{P}^{l}$ and $H=\mathbb{G}(\mathbf{V}) \times \mathbb{G}\left(\mathbf{V}^{\prime}\right)$. By Lemma $3, G$ acts transitively on $Y$. The condition $W=H \cdot W_{0}$ is clear. We have $G_{0}=\mathbf{U}^{l}$ and the embedding $W_{0} \hookrightarrow X_{0}$ can be identified using $\overleftarrow{p}_{\tilde{\mathbf{V}}}$, with $\mathbf{C}_{\mathfrak{n}}^{l, l^{\prime}} \hookrightarrow \mathbf{C}_{\mathfrak{n}}^{l}$. Note that by (15) we have $\mathfrak{Z}_{\mathfrak{m}, \mathfrak{m}^{\prime}}=\bar{W}$, and as before, $\bar{X}=\mathfrak{Y}_{\mathfrak{m}, \mathfrak{m}^{\prime}}$.

Remark 5 Let $p: \mathbf{C}_{\mathfrak{n}}^{l} \rightarrow \mathbf{C}_{\mathfrak{m}} \oplus \mathbf{C}_{\mathfrak{m}^{\prime}}$ be the restriction of $\overleftarrow{p}^{l}$ to $\mathbf{C}_{\mathfrak{n}}^{l}$. Thus, $p(\lambda)_{i, j}=\lambda_{i, j}$ for $(i, j) \in X_{\mathfrak{m}} \cup X_{\mathfrak{m}^{\prime}}$. Clearly, $p$ is $\mathbf{U}^{l}$-invariant, to wit, $\mathbf{U}^{l}$ acts on the fibers of $p$.

It is not difficult to see that $\operatorname{SG}\left(\mathfrak{m}, \mathfrak{m}^{\prime}\right)$ is also equivalent to each of the following conditions.

1. The set

$$
\left\{\left(\lambda, \lambda^{\prime}\right) \in \mathbf{C}_{\mathfrak{m}} \oplus \mathbf{C}_{\mathfrak{m}^{\prime}}: p^{-1}\left(\lambda, \lambda^{\prime}\right) \text { is a } \mathbf{U}^{l} \text {-orbit }\right\}
$$

is dense in $\mathbf{C}_{\mathfrak{m}} \oplus \mathbf{C}_{\mathfrak{m}^{\prime}}$.

2. The set

$$
\left\{(x, y) \in \mathfrak{C}_{\mathfrak{m}} \times \mathfrak{C}_{\mathfrak{m}^{\prime}}: \mathfrak{C}_{\mathfrak{n}} \cap p_{l}^{-1}(x, y) \text { is a } \mathbb{U}^{l} \text {-orbit }\right\}
$$

is dense in $\mathfrak{C}_{\mathfrak{m}} \times \mathfrak{C}_{\mathfrak{m}^{\prime}}$.

We will not give details since we will not use this result.

Remark 6 We could have defined an analogous condition $\overleftarrow{\mathrm{SG}}\left(\mathfrak{m}, \mathfrak{m}^{\prime}\right)$ to $\mathrm{SG}\left(\mathfrak{m}, \mathfrak{m}^{\prime}\right)$ by considering $\overleftarrow{\mathfrak{C}}_{\mathfrak{m}}$ instead of $\overrightarrow{\mathfrak{C}}_{\mathfrak{m}}$ (and similarly for other objects). Propositions 2 and 3 can be modified accordingly to yield that the conditions $\overleftarrow{\mathrm{SG}}\left(\mathfrak{m}, \mathfrak{m}^{\prime}\right)$ and $\mathrm{SG}\left(\mathfrak{m}^{\prime}, \mathfrak{m}\right)$ are equivalent.

\section{Corroborating evidence}

\subsection{Main evidence}

For convenience, let us say that a multisegment $\mathfrak{m}$ is good if for every multisegment $\mathfrak{m}^{\prime}$ the conditions $\mathrm{SA}\left(\mathfrak{m}, \mathfrak{m}^{\prime}\right)$ and $\operatorname{SG}\left(\mathfrak{m}, \mathfrak{m}^{\prime}\right)$ are equivalent and the conditions $\operatorname{SA}\left(\mathfrak{m}^{\prime}, \mathfrak{m}\right)$ and $\operatorname{SG}\left(\mathfrak{m}^{\prime}, \mathfrak{m}\right)$ are equivalent. Note that if $\mathfrak{m}$ is good 
and $Z(\mathfrak{m})$ is $\square$-irreducible, then for any $\mathfrak{m}^{\prime} \in \mathbb{N}(\Sigma)$ the irreducibility of $Z(\mathfrak{m}) \times Z\left(\mathfrak{m}^{\prime}\right)$ is equivalent to $\operatorname{IG}\left(\mathfrak{m}, \mathfrak{m}^{\prime}\right)$. By remark 3 , Conjecture 4 is equivalent to saying that every $\mathfrak{m}$ such that $Z(\mathfrak{m}) \in \operatorname{Irr}^{\square}$ is good, while Question 1 asks whether in fact every $\mathfrak{m} \in \mathbb{N}(\Sigma)$ is good.

Recall that a multisegment $\mathfrak{m}$ is called a (strict) ladder if it can be written as $\Delta_{1}+\cdots+\Delta_{k}$ where $\Delta_{i+1} \prec \Delta_{i}$ for all $i=1, \ldots, k-1$. This class is invariant under $\mathfrak{m} \mapsto \mathfrak{m}^{\#}$. The corresponding representations were studied in $[18,19]$. In particular, $G L S(\mathfrak{m})$ is satisfied and $Z(\mathfrak{m})$ is $\square$-irreducible for any ladder $\mathfrak{m}$.

The following result provides plenty of examples of good multisegments, in support of Conjecture 4.

Theorem 1 1. Every ladder multisegment is good.

2. Suppose that $\mathfrak{m}_{1}, \ldots, \mathfrak{m}_{k}$ are good multisegments and that $Z\left(\mathfrak{m}_{i}\right) \times Z\left(\mathfrak{m}_{j}\right)$ is irreducible for all $i, j$. Then $\mathfrak{m}=\mathfrak{m}_{1}+\cdots+\mathfrak{m}_{k}$ is good. (Recall that in this case, $Z(\mathfrak{m})=Z\left(\mathfrak{m}_{1}\right) \times \cdots \times Z\left(\mathfrak{m}_{k}\right)$ is $\square$-irreducible. $)$

We note that the class of representations in the second part of Theorem 1 is invariant under the Zelevinsky involution. (We do not know whether in general, $\mathfrak{m}$ is good $\Longleftrightarrow \mathfrak{m}^{\#}$ is good.) Also note that if both $\mathfrak{m}, \mathfrak{m}^{\prime}$ are ladders, then the condition $\operatorname{SG}\left(\mathfrak{m}, \mathfrak{m}^{\prime}\right)$ (and consequently, the irreducibility of $Z(\mathfrak{m}) \times Z\left(\mathfrak{m}^{\prime}\right)$ ) admits a very simple combinatorial description—see [19, Lemma 6.21].

Corollary 3 If $Z(\mathfrak{m})$ is unitarizable, then $\mathfrak{m}$ is good. Also, if every segment in $\mathfrak{m}$ (or $\mathfrak{m}^{\#}$ ) has the same length, then $\mathfrak{m}$ is good. In particular, if $Z(\mathfrak{m})$ is generic or unramified, then $\mathfrak{m}$ is good.

Indeed, the first part follows from Tadić classification [27] (see also [19]) which implies that if $Z(\mathfrak{m})$ is unitarizable, then we can write $\mathfrak{m}$ as a sum of multisegments

$$
\mathfrak{m}=\mathfrak{m}_{1}+\cdots+\mathfrak{m}_{k}
$$

where each $\mathfrak{m}_{i}$ is a ladder of the form $\mathfrak{m}_{i}=\Delta_{i, 1}+\cdots+\Delta_{i, r_{i}}$ where $\Delta_{i, j+1}=$ $\overleftarrow{\Delta}_{i, j}, j=1, \ldots, r_{i}-1$, and moreover $Z\left(\mathfrak{m}_{i}\right) \times Z\left(\mathfrak{m}_{j}\right)$ is irreducible for all $i, j$.

The second part follows from [21, Proposition 2.7].

Finally, if $Z(\mathfrak{m})$ is generic (resp., unramified), then every segment is $\mathfrak{m}$ $\left(\right.$ resp., $\left.\mathfrak{m}^{\#}\right)$ is a singleton.

Another case which will be useful later on is the following.

Example 2 Suppose that $\mathfrak{m}=\Delta_{1}+\cdots+\Delta_{k}$ and there exists $\rho \in \operatorname{Irr}_{c}$ such that $b\left(\Delta_{i}\right) \in\{\rho, \vec{\rho}\}$ for all $i$. Then, we can write $\mathfrak{m}=\mathfrak{m}_{1}+\cdots+\mathfrak{m}_{l}$ such that each $\mathfrak{m}_{i}$ is a ladder (consisting of either one or two segments) and $Z\left(\mathfrak{m}_{i}\right) \times Z\left(\mathfrak{m}_{j}\right)$ is irreducible for all $i, j$. (See Sect. 8.7 below.) Thus, $\mathfrak{m}$ is good. 
We will prove the theorem (along with all other results stated in this section) in Sect. 8 below.

\subsection{More precise statements}

The proof of Theorem 1 depends on several compatibility properties of the conditions $\mathrm{SA}\left(\mathfrak{m}, \mathfrak{m}^{\prime}\right)$ and $\mathrm{SG}\left(\mathfrak{m}, \mathfrak{m}^{\prime}\right)$ which are interesting in their own right. We formulate them in the following propositions, which provide additional attestation for Conjecture 4 as well as for an affirmative answer to Question 1.

Recall the notation max $\mathfrak{m}$ and $\mathfrak{m}^{-}$from Sect. 2.2.

Proposition 4 Suppose that $0 \neq \mathfrak{m}, \mathfrak{m}^{\prime} \in \mathbb{N}(\Sigma)$ with $\max \mathfrak{m}<\max \mathfrak{m}^{\prime}$. Then

1. $\mathrm{SA}\left(\mathfrak{m}, \mathfrak{m}^{\prime}\right)$ if and only if $\mathrm{SA}\left(\mathfrak{m}, \mathfrak{m}^{\prime-}\right)$ and $\left(\mathfrak{m}+\mathfrak{m}^{\prime}\right)^{-}=\mathfrak{m}+\mathfrak{m}^{\prime-}$.

2. $\operatorname{SG}\left(\mathfrak{m}, \mathfrak{m}^{\prime}\right)$ if and only if $\mathrm{SG}\left(\mathfrak{m}, \mathfrak{m}^{\prime-}\right)$ and $\left(\mathfrak{m}+\mathfrak{m}^{\prime}\right)^{-}=\mathfrak{m}+\mathfrak{m}^{\prime-}$.

Thus, Conjecture 4 and Question 1 reduce to the case where $\max \mathfrak{m}^{\prime} \leq \max \mathfrak{m}$.

The first part of Theorem 1 will follow from Proposition 4 and the following.

Proposition 5 Let $\mathfrak{m}=\sum_{i \in I} \Delta_{i}, \mathfrak{m}^{\prime}=\sum_{i^{\prime} \in I^{\prime}} \Delta_{i^{\prime}} \in \mathbb{N}(\Sigma)$. Assume that $I=I_{1} \cup I_{2}$ and $I^{\prime}=I_{1}^{\prime} \cup I_{2}^{\prime}$ (disjoint unions) and that

for any $i \in I_{1} \cup I_{1}^{\prime}$ and $j \in I_{2} \cup I_{2}^{\prime}$ we have $\Delta_{i} \nprec \Delta_{j}$,

for any $i \in I_{1} \cup I_{1}^{\prime}$ and $j \in I_{2} \cup I_{2}^{\prime}$ we have $\overleftarrow{\Delta}_{i} \nprec \Delta_{j}$

Let $\mathfrak{m}_{r}=\sum_{i \in I_{r}} \Delta_{i}$ and $\mathfrak{m}_{r}^{\prime}=\sum_{i^{\prime} \in I_{r}^{\prime}} \Delta_{i^{\prime}}, r=1,2$. Then,

1. $\mathrm{SA}\left(\mathfrak{m}, \mathfrak{m}^{\prime}\right)$ implies $\mathrm{SA}\left(\mathfrak{m}_{1}, \mathfrak{m}_{1}^{\prime}\right)$ and $\mathrm{SA}\left(\mathfrak{m}_{2}, \mathfrak{m}_{2}^{\prime}\right)$.

2. If $Z(\mathfrak{m}) \times Z\left(\mathfrak{m}^{\prime}\right)$ is irreducible, then $Z\left(\mathfrak{m}_{1}\right) \times Z\left(\mathfrak{m}_{1}^{\prime}\right)$ and $Z\left(\mathfrak{m}_{2}\right) \times Z\left(\mathfrak{m}_{2}^{\prime}\right)$ are irreducible.

3. $\operatorname{SG}\left(\mathfrak{m}, \mathfrak{m}^{\prime}\right)$ implies $\mathrm{SG}\left(\mathfrak{m}_{1}, \mathfrak{m}_{1}^{\prime}\right)$ and $\mathrm{SG}\left(\mathfrak{m}_{2}, \mathfrak{m}_{2}^{\prime}\right)$.

4. $G L S(\mathfrak{m})$ implies $G L S\left(\mathfrak{m}_{1}\right)$ and $G L S\left(\mathfrak{m}_{2}\right)$.

Extending the partial order on $\operatorname{Irr}_{c}$ lexicographically to $\Sigma$ (see Sect. 7.3) we infer

Corollary 4 For any two multisegments $\mathfrak{m}=\sum_{i \in I} \Delta_{i}, \mathfrak{m}^{\prime}=\sum_{i^{\prime} \in I^{\prime}} \Delta_{i^{\prime}}$ and $\Delta \in \Sigma$ we have

1. $\mathrm{SA}\left(\mathfrak{m}, \mathfrak{m}^{\prime}\right) \Longrightarrow \mathrm{SA}\left(\sum_{i \in I: \Delta_{i} \geq \Delta} \Delta_{i}, \sum_{i^{\prime} \in I^{\prime}: \Delta_{i^{\prime}} \geq \Delta} \Delta_{i^{\prime}}\right)$.

2. $\mathrm{SG}\left(\mathfrak{m}, \mathfrak{m}^{\prime}\right) \Longrightarrow \mathrm{SG}\left(\sum_{i \in I: \Delta_{i} \geq \Delta} \Delta_{i}, \sum_{i^{\prime} \in I^{\prime}: \Delta_{i^{\prime}} \geq \Delta} \Delta_{i^{\prime}}\right)$.

3. $\mathrm{SA}\left(\mathfrak{m}, \mathfrak{m}^{\prime}\right) \Longrightarrow \mathrm{SA}\left(\sum_{i \in I: e\left(\Delta_{i}\right) \in \Delta} \Delta_{i}, \sum_{i^{\prime} \in I^{\prime}: e\left(\Delta_{i^{\prime}}\right) \in \Delta} \Delta_{i^{\prime}}\right)$.

4. $\mathrm{SG}\left(\mathfrak{m}, \mathfrak{m}^{\prime}\right) \Longrightarrow \mathrm{SG}\left(\sum_{i \in I: e\left(\Delta_{i}\right) \in \Delta} \Delta_{i}, \sum_{i^{\prime} \in I^{\prime}: e\left(\Delta_{i^{\prime}}\right) \in \Delta} \Delta_{i^{\prime}}\right)$.

5. $\mathrm{SA}\left(\mathfrak{m}, \mathfrak{m}^{\prime}\right) \Longrightarrow \mathrm{SA}\left(\sum_{i \in I: b\left(\Delta_{i}\right) \in \Delta} \Delta_{i}, \sum_{i^{\prime} \in I^{\prime}: b\left(\Delta_{i^{\prime}}\right) \in \Delta} \Delta_{i^{\prime}}\right)$. 
6. $\mathrm{SG}\left(\mathfrak{m}, \mathfrak{m}^{\prime}\right) \Longrightarrow \mathrm{SG}\left(\sum_{i \in I: b\left(\Delta_{i}\right) \in \Delta} \Delta_{i}, \sum_{i^{\prime} \in I^{\prime}: b\left(\Delta_{i^{\prime}}\right) \in \Delta} \Delta_{i^{\prime}}\right)$.

7. If $Z(\mathfrak{m})$ is $\square$-irreducible, then so are $Z\left(\sum_{i \in I: \Delta_{i} \geq \Delta} \Delta_{i}\right), Z\left(\sum_{i \in I: e\left(\Delta_{i}\right) \in \Delta} \Delta_{i}\right)$ and $Z\left(\sum_{i \in I: b\left(\Delta_{i}\right) \in \Delta} \Delta_{i}\right)$.

8. $G L S(\mathfrak{m})$ implies $G L S\left(\sum_{i \in I: \Delta_{i} \geq \Delta} \Delta_{i}\right), \quad G L S\left(\sum_{i \in I: e\left(\Delta_{i}\right) \in \Delta} \Delta_{i}\right)$ and $G L S\left(\sum_{i \in I: b\left(\Delta_{i}\right) \in \Delta} \Delta_{i}\right)$.

The second part of Theorem 1 follows from the following more precise statement.

Proposition 6 Let $\mathfrak{m}, \mathfrak{m}^{\prime}, \mathfrak{n} \in \mathbb{N}(\Sigma)$. Then,

1. $\mathrm{SA}\left(\mathfrak{m}, \mathfrak{m}^{\prime}\right)$ and $\mathrm{SA}\left(\mathfrak{m}+\mathfrak{m}^{\prime}, \mathfrak{n}\right)$ imply $\mathrm{SA}\left(\mathfrak{m}, \mathfrak{m}^{\prime}+\mathfrak{n}\right)$.

2. $\mathrm{SG}\left(\mathfrak{m}, \mathfrak{m}^{\prime}\right)$ and $\mathrm{SG}\left(\mathfrak{m}+\mathfrak{m}^{\prime}, \mathfrak{n}\right)$ imply $\mathrm{SG}\left(\mathfrak{m}, \mathfrak{m}^{\prime}+\mathfrak{n}\right)$ and $\mathrm{SG}(\mathfrak{m}, \mathfrak{n})$.

3. $\mathrm{SG}\left(\mathfrak{m}, \mathfrak{m}^{\prime}\right)$ and $\mathrm{SG}(\mathfrak{m}, \mathfrak{n})$ imply $\mathrm{SG}\left(\mathfrak{m}, \mathfrak{m}^{\prime}+\mathfrak{n}\right)$.

4. Suppose that $\mathrm{SG}\left(\mathfrak{m}, \mathfrak{m}^{\prime}\right)$ and $\mathrm{SG}\left(\mathfrak{m}^{\prime}, \mathfrak{m}\right)$. Then

$$
\mathrm{SG}\left(\mathfrak{m}+\mathfrak{m}^{\prime}, \mathfrak{n}\right) \Longleftrightarrow \mathrm{SG}(\mathfrak{m}, \mathfrak{n}) \text { and } \mathrm{SG}\left(\mathfrak{m}^{\prime}, \mathfrak{n}\right) \text {. }
$$

5. Suppose that $Z(\mathfrak{m})$ and $Z\left(\mathfrak{m}^{\prime}\right)$ are $\square$-irreducible and $Z(\mathfrak{m}) \times Z\left(\mathfrak{m}^{\prime}\right)$ is irreducible. Then

$$
\mathrm{SA}\left(\mathfrak{m}+\mathfrak{m}^{\prime}, \mathfrak{n}\right) \Longleftrightarrow \mathrm{SA}(\mathfrak{m}, \mathfrak{n}) \text { and } \mathrm{SA}\left(\mathfrak{m}^{\prime}, \mathfrak{n}\right) .
$$

By passing to the contragredient, similar statements hold for the relations obtained from SA or SG by switching the arguments.

Note that the statements of the proposition are not completely symmetric in SA and SG. It would be interesting to show the missing representationtheoretic counterparts, namely

$$
\begin{aligned}
& \mathrm{SA}\left(\mathfrak{m}, \mathfrak{m}^{\prime}\right) \text { and } \mathrm{SA}\left(\mathfrak{m}+\mathfrak{m}^{\prime}, \mathfrak{n}\right) \Longrightarrow \operatorname{SA}(\mathfrak{m}, \mathfrak{n}), \\
& \operatorname{SA}\left(\mathfrak{m}, \mathfrak{m}^{\prime}\right) \text { and } \operatorname{SA}(\mathfrak{m}, \mathfrak{n}) \Longrightarrow \operatorname{SA}\left(\mathfrak{m}, \mathfrak{m}^{\prime}+\mathfrak{n}\right)
\end{aligned}
$$

at least when $Z(\mathfrak{m}), Z\left(\mathfrak{m}^{\prime}\right)$ and $Z(\mathfrak{n})$ are $\square$-irreducible.

\subsection{Further evidence}

The following compatibility result is motivated by (13).

Proposition 7 Let $\mathfrak{m}, \mathfrak{m}^{\prime} \in \mathbb{N}(\Sigma)$ and $\mathfrak{n}=\mathfrak{m}+\mathfrak{m}^{\prime}$.

1. Suppose that $Z(\mathfrak{m}), Z\left(\mathfrak{m}^{\prime}\right)$ are $\square$-irreducible and the conditions $\operatorname{SA}\left(\mathfrak{m}, \mathfrak{m}^{\prime}\right)$ and $\mathrm{SA}(\mathfrak{n}, \mathfrak{m})$ are satisfied. Then, $Z(\mathfrak{n})$ is $\square$-irreducible.

2. Suppose that the conditions $G L S(\mathfrak{m}), G L S\left(\mathfrak{m}^{\prime}\right), S G\left(\mathfrak{m}, \mathfrak{m}^{\prime}\right)$ and $\mathrm{SG}(\mathfrak{n}, \mathfrak{m})$ are satisfied. Then, GLS(n) holds. 
For the last consistency check that we will state here, we fix $\rho \in \operatorname{Irr}_{c}$. For $\pi \in$ Irr we write $\rho\left\lceil\pi\right.$ if there does not exist $\pi^{\prime} \in \operatorname{Irr}$ such that $\pi \hookrightarrow \rho \times \pi^{\prime}$. (A more general notation will be introduced in Sect. 7.2 below.) For any $\pi=Z(\mathfrak{m}) \in$ Irr there exist a unique integer $m \geq 0$ and a unique $\pi^{\prime}=Z\left(\mathfrak{m}^{\prime}\right) \in$ Irr such that $\pi \hookrightarrow \overbrace{\rho \times \cdots \times \rho}^{m} \times \pi^{\prime}$ and $\rho \ltimes \pi^{\prime}$. We denote $\rho \mathfrak{m}=\mathfrak{m}^{\prime}$.

Proposition 8 Let $\mathfrak{m}, \mathfrak{m}^{\prime} \in \mathbb{N}(\Sigma), \rho \in \operatorname{Irr}_{c}$ and assume that $\rho \preccurlyeq Z\left(\mathfrak{m}^{\prime}\right)$. Let $\tilde{X}_{\mathfrak{m}, \mathfrak{m}^{\prime}}^{\rho}$ and $\tilde{Y}_{\mathfrak{m}, \mathfrak{m}^{\prime}}^{\rho}$ be as in (32) below. Then

1. $\mathrm{SA}\left(\mathfrak{m}, \mathfrak{m}^{\prime}\right) \Longleftrightarrow \operatorname{SA}_{\rho}\left(\mathfrak{m}, \mathfrak{m}^{\prime}\right)$ and $\# \tilde{X}_{\mathfrak{m}, \mathfrak{m}^{\prime}}^{\rho}=\# \tilde{Y}_{\mathfrak{m}, \mathfrak{m}^{\prime}}^{\rho}$.

2. $\mathrm{SG}\left(\mathfrak{m}, \mathfrak{m}^{\prime}\right) \Longleftrightarrow \mathrm{SG}\left({ }_{\rho} \mathfrak{m}, \mathfrak{m}^{\prime}\right)$ and $\# \tilde{X}_{\mathfrak{m}, \mathfrak{m}^{\prime}}^{\rho}=\# \tilde{Y}_{\mathfrak{m}, \mathfrak{m}^{\prime}}^{\rho}$.

\section{Some preparation}

\subsection{Jacquet modules}

For any $n, m \geq 0$ let $J_{n, m}: \mathcal{M}_{n+m} \rightarrow \mathcal{M}_{n} \otimes \mathcal{M}_{m}$ be the Jacquet functor with respect to the standard parabolic subgroup of type $(n, m)$ where we identify $\mathcal{M}_{n} \otimes \mathcal{M}_{m}$ with the category of finite-length representations of $\mathrm{GL}_{n}(F) \times$ $\mathrm{GL}_{m}(F) .{ }^{3}$ We obtain the "total" Jacquet functor

$$
J=\oplus_{n, m \geq 0} J_{n, m}: \mathcal{M} \rightarrow \mathcal{M} \otimes \mathcal{M} .
$$

On the level of the Grothendieck group, this gives rise to a ring homomorphism

$$
J_{\mathrm{ss}}: \mathcal{R} \rightarrow \mathcal{R} \otimes \mathcal{R}
$$

For instance, for any segment $\Delta=\left\{\rho_{1}, \ldots, \rho_{k}\right\}$ with $\rho_{i+1}=\vec{\rho}_{i}, i=$ $1, \ldots, k-1$ we have

$$
\begin{aligned}
& J(Z(\Delta))=\oplus_{l=0}^{k} Z\left(\left\{\rho_{1}, \ldots, \rho_{l}\right\}\right) \otimes Z\left(\left\{\rho_{l+1}, \ldots, \rho_{k}\right\}\right), \\
& J(L(\Delta))=\oplus_{l=0}^{k} L\left(\left\{\rho_{l+1}, \ldots, \rho_{k}\right\}\right) \otimes L\left(\left\{\rho_{1}, \ldots, \rho_{l}\right\}\right) .
\end{aligned}
$$

By Frobenius reciprocity, for any $\pi, \pi^{\prime} \in \mathcal{M}$ we have a canonical functorial surjection [5]

$$
\mathfrak{p}_{\pi, \pi^{\prime}}: J\left(\pi \times \pi^{\prime}\right) \rightarrow \pi \otimes \pi^{\prime}
$$

\footnotetext{
3 The tensor product of categories was defined by Deligne in [8].
} 
For any $\mathfrak{s} \in \mathbb{N}\left(\operatorname{Irr}_{c}\right)$ let $\mathcal{M}_{\mathfrak{s}}$ be the Serre subcategory of $\mathcal{M}$ consisting of representations all of whose irreducible subquotients have supercuspidal support $\mathfrak{s}$. We have

$$
\mathcal{M}=\oplus_{\mathfrak{s} \in \mathbb{N}\left(\operatorname{Irr}_{c}\right)} \mathcal{M}_{\mathfrak{s}}
$$

and $\mathcal{M}_{\mathfrak{s}} \times \mathcal{M}_{\mathfrak{s}^{\prime}} \subset \mathcal{M}_{\mathfrak{s}+\mathfrak{s}^{\prime}}$ for any $\mathfrak{s}, \mathfrak{s}^{\prime} \in \mathbb{N}\left(\operatorname{Irr}_{c}\right)$. For any $\pi \in \mathcal{M}$ and $\mathfrak{s} \in \mathbb{N}\left(\operatorname{Irr}_{c}\right)$ we denote by $\pi_{\mathfrak{s}}$ the $\mathfrak{s}$-component of $\pi$ with the respect to the decomposition (22). Thus,

$$
\pi=\oplus_{\mathfrak{s} \in \mathbb{N}\left(\operatorname{Irr}_{c}\right)} \pi_{\mathfrak{s}} .
$$

Similarly, for any $\Pi \in \mathcal{M} \otimes \mathcal{M}$ and $\mathfrak{s}, \mathfrak{s}^{\prime} \in \mathbb{N}\left(\operatorname{Irr}_{c}\right)$ we denote by $\Pi_{\mathfrak{s} \otimes \mathfrak{s}^{\prime}}$ the $\mathfrak{s} \otimes \mathfrak{s}^{\prime}$-component of $\Pi$ with the respect to the decomposition

$$
\mathcal{M} \otimes \mathcal{M}=\oplus_{\mathfrak{s}, \mathfrak{s}^{\prime} \in \mathbb{N}\left(\operatorname{Irr}_{c}\right)} \mathcal{M}_{\mathfrak{s}} \otimes \mathcal{M}_{\mathfrak{s}^{\prime}}
$$

Remark 7 Let $\pi \in \mathcal{M}, \mathfrak{s} \in \mathbb{N}\left(\operatorname{Irr}_{c}\right)$ and $\tau \in \mathcal{M}_{\mathfrak{s}}$. Any morphism $p: \pi \rightarrow \tau$ factor through a morphism $\pi_{\mathfrak{s}} \rightarrow \tau$. Suppose that $p$ is surjective. Then, the restriction $p^{\mathfrak{s}}$ of $p$ to $\pi_{\mathfrak{s}}$ is surjective. Moreover, the following conditions are equivalent.

1. $p^{\mathfrak{s}}$ is an isomorphism.

2. $\pi_{\mathfrak{s}} \simeq \tau$.

3. $\operatorname{JH}\left(\pi_{\mathfrak{s}}\right)=\mathrm{JH}(\tau)$.

4. $\mathrm{JH}\left(\pi_{\mathfrak{s}}\right) \leq \mathrm{JH}(\tau)$ in $\mathbb{N}(\mathrm{Irr})$.

In this case, we say that $p$ is a component map, or that (somewhat informally) $\tau$ "is" the $\mathfrak{s}$-component of $\pi$. Similar terminology will apply for $\mathcal{M} \otimes \mathcal{M}$ and $\mathfrak{s} \otimes \mathfrak{s}^{\prime}$ with $\mathfrak{s}, \mathfrak{s}^{\prime} \in \mathbb{N}\left(\operatorname{Irr}_{C}\right)$.

\subsection{Separated representations}

The following technical definition will be useful.

Definition 6 Suppose that $\pi \in \mathcal{M}_{\mathfrak{s}}$ and $\pi^{\prime} \in \mathcal{M}_{\mathfrak{s}^{\prime}}$ for some $\mathfrak{s}, \mathfrak{s}^{\prime} \in \mathbb{N}\left(\operatorname{Irr}_{c}\right)$. We write $\pi \gtrless \pi^{\prime}$ and say that $\pi$ is left-separated from $\pi^{\prime}$ if $\mathfrak{p}_{\pi, \pi^{\prime}}$ is a component map, i.e., if the following equivalent conditions are satisfied.

1. The map $\mathfrak{p}_{\pi, \pi^{\prime}}$ factors through an isomorphism $J\left(\pi \times \pi^{\prime}\right)_{\mathfrak{s} \otimes \mathfrak{s}^{\prime}} \rightarrow \pi \otimes \pi^{\prime}$.

2. $J\left(\pi \times \pi^{\prime}\right)_{\mathfrak{s} \otimes \mathfrak{s}^{\prime}} \simeq \pi \otimes \pi^{\prime}$.

3. $\mathrm{JH}\left(J\left(\pi \times \pi^{\prime}\right)_{\mathfrak{s} \otimes \mathfrak{s}^{\prime}}\right)=\mathrm{JH}\left(\pi \otimes \pi^{\prime}\right)\left(=\mathrm{JH}(\pi) \otimes \mathrm{JH}\left(\pi^{\prime}\right)\right)$.

4. $\mathrm{JH}\left(J\left(\pi \times \pi^{\prime}\right)_{\mathfrak{s} \otimes \mathfrak{s}^{\prime}}\right) \leq \mathrm{JH}\left(\pi \otimes \pi^{\prime}\right)$ in $\mathbb{N}(\operatorname{Irr} \times \operatorname{Irr})$.

As the notation suggests, the relation $\Uparrow$ is not symmetric. 
Example 3 Suppose that $\rho \in \operatorname{Irr}_{c}$ and $\pi \in \operatorname{Irr}$. Then

$$
\begin{aligned}
\rho \ltimes \pi & \Longleftrightarrow \nexists \pi^{\prime} \in \text { Irr such that } \pi \hookrightarrow \rho \times \pi^{\prime} \\
& \Longleftrightarrow \nexists \pi^{\prime} \in \mathcal{M} \text { such that } \pi \hookrightarrow \rho \times \pi^{\prime} .
\end{aligned}
$$

The following easy property will be used repeatedly.

Lemma 4 Suppose that $\pi \in \mathcal{M}_{\mathfrak{s}}$ and $\pi^{\prime} \in \mathcal{M}_{\mathfrak{s}^{\prime}}$ for some $\mathfrak{s}, \mathfrak{s}^{\prime} \in \mathbb{N}\left(\operatorname{Irr}_{c}\right)$. If $\pi \ll \pi^{\prime}$, then $\tau \Uparrow \tau^{\prime}$ for any subquotient $\tau$ of $\pi$ and $\tau^{\prime}$ of $\pi^{\prime}$. Conversely, if $\tau \ltimes \tau^{\prime}$ for every $\tau \in \mathrm{JH}(\pi)$ and $\tau^{\prime} \in \mathrm{JH}\left(\pi^{\prime}\right)$, then $\pi \ll \pi^{\prime}$.

Proof Suppose that we have a short exact sequence

$$
0 \rightarrow \sigma \rightarrow \pi \rightarrow \tau \rightarrow 0
$$

Then, we have a commutative diagram

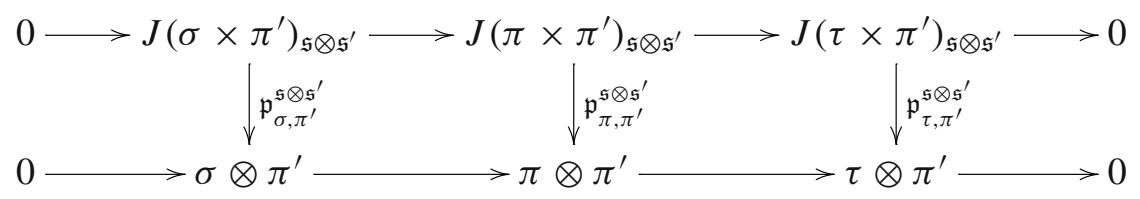

where the rows are exact and the vertical morphisms are surjective. It follows that $\pi \gtrless \pi^{\prime}$ if and only if $\sigma \gtrless \pi^{\prime}$ and $\tau \Uparrow \pi^{\prime}$. A similar statement holds for a short exact sequence

$$
0 \rightarrow \sigma^{\prime} \rightarrow \pi^{\prime} \rightarrow \tau^{\prime} \rightarrow 0
$$

The lemma immediately follows.

We will use two additional properties of the relation $§$.

Lemma 5 Let $\pi, \pi^{\prime} \in \operatorname{Irr}$ and suppose that $\pi \times \pi^{\prime}$. Then, $\pi \times \pi^{\prime}$ is SI and

$$
J\left(\operatorname{soc}\left(\pi \times \pi^{\prime}\right)\right)_{\mathfrak{s}(\pi) \otimes \mathfrak{s}\left(\pi^{\prime}\right)}=\pi \otimes \pi^{\prime} .
$$

Moreover, up to isomorphism, $\operatorname{soc}\left(\pi \times \pi^{\prime}\right)$ is the only irreducible subquotient $\sigma$ of $\pi \times \pi^{\prime}$ such that $J(\sigma)_{\mathfrak{s}(\pi) \otimes \mathfrak{s}\left(\pi^{\prime}\right)} \neq 0$.

Proof If $\sigma$ is a non-zero subrepresentation of $\pi \times \pi^{\prime}$, then by Frobenius reciprocity $J(\sigma) \rightarrow \pi \otimes \pi^{\prime}$. Since $\pi \ll \pi^{\prime}$ it follows that $J(\sigma)_{\mathfrak{s}(\pi) \otimes \mathfrak{s}\left(\pi^{\prime}\right)}=$ $J\left(\pi \times \pi^{\prime}\right)_{\mathfrak{s}(\pi) \otimes \mathfrak{s}\left(\pi^{\prime}\right)}=\pi \otimes \pi^{\prime}$. The statements now follow from the exactness of $J$. 
Lemma 6 Suppose that $\pi \in \mathcal{M}_{\mathfrak{s}}, \pi^{\prime} \in \mathcal{M}_{\mathfrak{s}^{\prime}}$ and $\pi \Uparrow \pi^{\prime}$. Then,

$$
\operatorname{soc}\left(\pi \times \pi^{\prime}\right)=\operatorname{soc}\left(\operatorname{soc}(\pi) \times \operatorname{soc}\left(\pi^{\prime}\right)\right) .
$$

Proof Clearly, $\operatorname{soc}\left(\operatorname{soc}(\pi) \times \operatorname{soc}\left(\pi^{\prime}\right)\right) \hookrightarrow \operatorname{soc}\left(\pi \times \pi^{\prime}\right)$. Let $\tau$ be an irreducible subrepresentation of $\pi \times \pi^{\prime}$ and suppose on the contrary that $\tau$ is not a subrepresentation of $\operatorname{soc}(\pi) \times \operatorname{soc}\left(\pi^{\prime}\right)$. Then $\tau \cap\left(\operatorname{soc}(\pi) \times \operatorname{soc}\left(\pi^{\prime}\right)\right)=0$, and by the exactness of $J$ we have $J(\tau) \cap J\left(\operatorname{soc}(\pi) \times \operatorname{soc}\left(\pi^{\prime}\right)\right)=0$. In particular,

$$
J(\tau)_{\mathfrak{s} \otimes \mathfrak{s}^{\prime}} \cap J\left(\operatorname{soc}(\pi) \times \operatorname{soc}\left(\pi^{\prime}\right)\right)_{\mathfrak{s} \otimes \mathfrak{s}^{\prime}}=0 .
$$

On the other hand, we claim that

$$
J\left(\operatorname{soc}(\pi) \times \operatorname{soc}\left(\pi^{\prime}\right)\right)_{\mathfrak{s} \otimes \mathfrak{s}^{\prime}}=\operatorname{soc}\left(J\left(\pi \times \pi^{\prime}\right)_{\mathfrak{s} \otimes \mathfrak{s}^{\prime}}\right) .
$$

Indeed, since $\pi \times \pi^{\prime}$, the restriction of $\mathfrak{p}_{\pi, \pi^{\prime}}$ to $J\left(\pi \times \pi^{\prime}\right)_{\mathfrak{s} \otimes \mathfrak{s}^{\prime}}$ is an isomorphism. It is therefore enough to check that the image of $J\left(\operatorname{soc}(\pi) \times \operatorname{soc}\left(\pi^{\prime}\right)\right)_{\mathfrak{s} \otimes \mathfrak{s}^{\prime}}$ under $\mathfrak{p}_{\pi, \pi^{\prime}}$ is $\operatorname{soc}\left(\pi \otimes \pi^{\prime}\right)=\operatorname{soc}(\pi) \otimes \operatorname{soc}\left(\pi^{\prime}\right)$, and this holds since $\operatorname{soc}(\pi) \Uparrow \operatorname{soc}\left(\pi^{\prime}\right)$ by Lemma 4 .

It follows from (24) and (25) that $J(\tau)_{\mathfrak{s} \otimes \mathfrak{s}^{\prime}}=0$ since any non-zero subrepresentation of $J\left(\pi \times \pi^{\prime}\right)_{\mathfrak{s} \otimes \mathfrak{s}^{\prime}}$ must intersect its socle nontrivially. However, by Frobenius reciprocity $\operatorname{Hom}\left(J(\tau), \pi \otimes \pi^{\prime}\right) \neq 0$ and hence $J(\tau)_{\mathfrak{s} \otimes \mathfrak{s}^{\prime}} \neq 0$. We get a contradiction.

Corollary 5 If $\pi \in \mathcal{M}_{\mathfrak{s}}, \pi^{\prime} \in \mathcal{M}_{\mathfrak{s}^{\prime}}$ are SI and $\pi \ll \pi^{\prime}$, then $\pi \times \pi^{\prime}$ is SI and $\operatorname{soc}\left(\pi \times \pi^{\prime}\right)=\operatorname{soc}\left(\operatorname{soc}(\pi) \times \operatorname{soc}\left(\pi^{\prime}\right)\right)$. Moreover, $\operatorname{soc}(\pi) \otimes \operatorname{soc}\left(\pi^{\prime}\right) \unlhd$ $J\left(\pi \times \pi^{\prime}\right)$.

Indeed, $\operatorname{soc}(\pi) \otimes \operatorname{soc}\left(\pi^{\prime}\right) \leq J\left(\operatorname{soc}\left(\operatorname{soc}(\pi) \times \operatorname{soc}\left(\pi^{\prime}\right)\right)\right)$ by Frobenius reciprocity, while $\operatorname{soc}(\pi) \otimes \operatorname{soc}\left(\pi^{\prime}\right) \unlhd J\left(\pi \times \pi^{\prime}\right)$ since $\operatorname{soc}(\pi) \otimes \operatorname{soc}\left(\pi^{\prime}\right) \unlhd$ $J\left(\pi \times \pi^{\prime}\right)_{\mathfrak{s} \otimes \mathfrak{s}^{\prime}} \simeq \pi \otimes \pi^{\prime}$ by assumption.

\subsection{A simple criterion for separation}

Recall that we fixed a total order $\leq$ on $\operatorname{Irr}_{c}$ subject only to the condition that $\rho<\vec{\rho}$ for all $\rho \in \operatorname{Irr}_{c}$. We extend this lexicographically to a total order on $\Sigma$ (also denoted by $\leq$ ), that is, $\Delta \leq \Delta^{\prime}$ iff either $e(\Delta)<e\left(\Delta^{\prime}\right)$ or $e(\Delta)=e\left(\Delta^{\prime}\right)$ and $b(\Delta) \leq b\left(\Delta^{\prime}\right)$. Clearly, if $\Delta \prec \Delta^{\prime}$ (resp., $\overleftarrow{\Delta} \prec \Delta^{\prime}$ ) then $\Delta<\Delta^{\prime}$ (resp., $\Delta \leq \Delta^{\prime}$ ). Note that if $e(\Delta)=e\left(\Delta^{\prime}\right)$ then $\Delta \leq \Delta^{\prime}$ if and only if $\Delta \supseteq \Delta^{\prime}$. (In order to avoid confusion, we will never consider $\Sigma$ as a poset with respect by inclusion.)

If $\mathfrak{m}=\sum_{i \in I} \Delta_{i} \in \mathbb{N}(\Sigma)$ and $\Delta \in \Sigma$, then we write $\mathfrak{m}_{\geq \Delta}=\sum_{i \in I: \Delta_{i} \geq \Delta} \Delta_{i}$ and similarly for $\mathfrak{m}_{<\Delta}$. 
Lemma 7 Let $\mathfrak{m}=\sum_{i \in I} \Delta_{i}, \mathfrak{m}^{\prime}=\sum_{i^{\prime} \in I^{\prime}} \Delta_{i^{\prime}} \in \mathbb{N}(\Sigma)$ with $I \cap I^{\prime}=\emptyset$.

Suppose that $\overleftarrow{\Delta}_{i} \nprec \Delta_{i^{\prime}}$ for all $i \in I, i^{\prime} \in I^{\prime}$. (For instance, this holds if there exists $\Delta \in \Sigma$ such that $\mathfrak{m}=\mathfrak{m}_{\geq \Delta}$ and $\mathfrak{m}^{\prime}=\mathfrak{m}_{<\Delta}^{\prime}$.) Then, $\mathfrak{z}(\mathfrak{m}) \ltimes \mathfrak{z}\left(\mathfrak{m}^{\prime}\right)$ and $\lambda\left(\mathfrak{m}^{\prime}\right)\lceil\lambda(\mathfrak{m})$.

Proof We show that $\mathfrak{z}(\mathfrak{m}) \preccurlyeq \mathfrak{z}\left(\mathfrak{m}^{\prime}\right)$, the other part being similar. Suppose on the contrary that this is not the case. Then, by the geometric lemma of BernsteinZelevinsky and (21), there exists for each $i \in I \cup I^{\prime}$ a partition $\Delta_{i}=A_{i} \cup B_{i}$ such that

- $\rho_{1}<\rho_{2}$ whenever $\rho_{1} \in A_{i}$ and $\rho_{2} \in B_{i}, i \in I \cup I^{\prime}$. In particular, $A_{i}, B_{i} \in \Sigma \cup\{\emptyset\}$.

$-0 \neq \sum_{i \in I} \operatorname{supp} B_{i}=\sum_{i^{\prime} \in I^{\prime}} \operatorname{supp} A_{i^{\prime}}$ and in particular $\cup_{i^{\prime} \in I^{\prime}} A_{i^{\prime}}=$ $\cup_{i \in I} B_{i} \neq \varnothing$.

Let

$$
\rho=\min \cup_{i \in I} B_{i}=\min \cup_{i^{\prime} \in I^{\prime}} A_{i^{\prime}}
$$

and let $i \in I$ be such that $\rho \in B_{i}$. Then, $e\left(\Delta_{i}\right) \in B_{i}$ and therefore there exists $i^{\prime} \in I^{\prime}$ such that $e\left(\Delta_{i}\right) \in A_{i^{\prime}}$. Thus, $b\left(\Delta_{i^{\prime}}\right) \in A_{i^{\prime}}$ and we infer that $b\left(\Delta_{i^{\prime}}\right) \geq \rho$ by the minimality of $\rho$. Hence, $\overleftarrow{B}_{i} \prec A_{i^{\prime}}$ (cf. (3)) and therefore $\overleftarrow{\Delta}_{i} \prec \Delta_{i^{\prime}}$, in contradiction to our assumption.

Corollary 6 Suppose that $\pi \in \mathcal{M}_{\mathfrak{s}}, \pi^{\prime} \in \mathcal{M}_{\mathfrak{s}^{\prime}}$ and there exists $\Delta \in \Sigma$ such that each irreducible subquotient $Z(\mathfrak{m})$ of $\pi$ (resp., $\pi^{\prime}$ ) satisfies $\mathfrak{m}=$ $\mathfrak{m}_{\geq \Delta}$ (resp., $\left.\mathfrak{m}=\mathfrak{m}_{<\Delta}\right)$. Then, $\pi \mathbb{R} \pi^{\prime}$. A similar conclusion holds if each irreducible subquotient $L(\mathfrak{m})$ of $\pi$ (resp., $\left.\pi^{\prime}\right)$ satisfies $\mathfrak{m}=\mathfrak{m}_{<\Delta}$ (resp., $\mathfrak{m}=$ $\mathfrak{m}_{\geq \Delta}$ ).

\section{Proofs}

In this section we prove the statements of Sect. 6.

For the properties pertaining to the geometric condition $\mathrm{SG}\left(\mathfrak{m}, \mathfrak{m}^{\prime}\right)$ we will mostly use the last and most tangible criterion of Proposition 3. It is likely, however, that the proofs can be made more conceptual.

When writing a multisegment as $\mathfrak{m}=\sum_{i \in I} \Delta_{i}$ it will sometimes be convenient to allow $\Delta_{i}=\emptyset$ for some indices. These inconsequential indices will not have any effect on $\mathfrak{m}$ or on the objects pertaining to it (such as $X_{\mathfrak{m}}, Y_{\mathfrak{m}}$ of Sect. 4.3). We will use this convention throughout. 


\subsection{Proof of Proposition 5 and Corollary 4}

Let $\pi=Z(\mathfrak{m}), \pi^{\prime}=Z\left(\mathfrak{m}^{\prime}\right), \pi_{j}=Z\left(\mathfrak{m}_{j}\right), \pi_{j}^{\prime}=Z\left(\mathfrak{m}_{j}^{\prime}\right), j=1,2$. Then, by (10) and (20a), $\pi=\operatorname{soc}\left(\pi_{1} \times \pi_{2}\right)$ and $\pi^{\prime}=\operatorname{soc}\left(\pi_{1}^{\prime} \times \pi_{2}^{\prime}\right)$. Thus $J(\pi) \rightarrow$ $\pi_{1} \otimes \pi_{2}$ and $J\left(\pi^{\prime}\right) \rightarrow \pi_{1}^{\prime} \otimes \pi_{2}^{\prime}$. By the geometric lemma, it follows that $J\left(\pi \times \pi^{\prime}\right)$ admits $\pi_{1} \times \pi_{1}^{\prime} \otimes \pi_{2} \times \pi_{2}^{\prime}$ as a subquotient. On the other hand by (20b) and Lemma $7, \pi_{1} \times \pi_{1}^{\prime} \ltimes \pi_{2} \times \pi_{2}^{\prime}$ and hence

$$
J\left(\pi_{1} \times \pi_{1}^{\prime} \times \pi_{2} \times \pi_{2}^{\prime}\right)_{\mathfrak{s}\left(\pi_{1}\right)+\mathfrak{s}\left(\pi_{1}^{\prime}\right) \otimes \mathfrak{s}\left(\pi_{2}\right)+\mathfrak{s}\left(\pi_{2}^{\prime}\right)}=\pi_{1} \times \pi_{1}^{\prime} \otimes \pi_{2} \times \pi_{2}^{\prime} .
$$

Since $\mathrm{JH}\left(\pi_{1} \times \pi_{1}^{\prime} \times \pi_{2} \times \pi_{2}^{\prime}\right)=\mathrm{JH}\left(\pi_{1} \times \pi_{2} \times \pi_{1}^{\prime} \times \pi_{2}^{\prime}\right)$, we infer that

$$
J\left(\pi \times \pi^{\prime}\right)_{\mathfrak{s}\left(\pi_{1}\right)+\mathfrak{s}\left(\pi_{1}^{\prime}\right) \otimes \mathfrak{s}\left(\pi_{2}\right)+\mathfrak{s}\left(\pi_{2}^{\prime}\right)}=\pi_{1} \times \pi_{1}^{\prime} \otimes \pi_{2} \times \pi_{2}^{\prime} .
$$

Moreover, $Z\left(\mathfrak{m}_{1}+\mathfrak{m}_{1}^{\prime}\right) \Uparrow Z\left(\mathfrak{m}_{2}+\mathfrak{m}_{2}^{\prime}\right)$ (since $\left.\pi_{1} \times \pi_{1}^{\prime} \lessdot \pi_{2} \times \pi_{2}^{\prime}\right)$ and $\mathrm{SA}\left(\mathfrak{m}_{1}+\mathfrak{m}_{1}^{\prime}, \mathfrak{m}_{2}+\mathfrak{m}_{2}^{\prime}\right)$ (by (20a) and (10)). Hence, by Lemma 5

$$
J\left(Z\left(\mathfrak{m}+\mathfrak{m}^{\prime}\right)\right)_{\mathfrak{s}\left(\pi_{1}\right)+\mathfrak{s}\left(\pi_{1}^{\prime}\right) \otimes \mathfrak{s}\left(\pi_{2}\right)+\mathfrak{s}\left(\pi_{2}^{\prime}\right)}=Z\left(\mathfrak{m}_{1}+\mathfrak{m}_{1}^{\prime}\right) \otimes Z\left(\mathfrak{m}_{2}+\mathfrak{m}_{2}^{\prime}\right)
$$

The first two parts of Proposition 5 readily follow from (26) and (27). Indeed, if $Z\left(\mathfrak{m}+\mathfrak{m}^{\prime}\right) \hookrightarrow \pi \times \pi^{\prime}$, then

$$
Z\left(\mathfrak{m}_{1}+\mathfrak{m}_{1}^{\prime}\right) \otimes Z\left(\mathfrak{m}_{2}+\mathfrak{m}_{2}^{\prime}\right) \hookrightarrow \pi_{1} \times \pi_{1}^{\prime} \otimes \pi_{2} \times \pi_{2}^{\prime},
$$

while if $\pi \times \pi^{\prime}$ is irreducible (in which case $\pi \times \pi^{\prime}=Z\left(\mathfrak{m}+\mathfrak{m}^{\prime}\right)$ ) then

$$
Z\left(\mathfrak{m}_{1}+\mathfrak{m}_{1}^{\prime}\right) \otimes Z\left(\mathfrak{m}_{2}+\mathfrak{m}_{2}^{\prime}\right)=\pi_{1} \times \pi_{1}^{\prime} \otimes \pi_{2} \times \pi_{2}^{\prime}
$$

as required.

To prove the third part, let $\lambda \in \mathbf{C}_{\mathfrak{m}}$ and $\lambda^{\prime} \in \mathbf{C}_{\mathfrak{m}^{\prime}}$. For $r=1$, 2, let $\tilde{\lambda} \in \mathbf{C}_{\mathfrak{m}_{r}}$ be such that $\tilde{\lambda}_{i, j}=\lambda_{i, j}$ for all $(i, j) \in X_{\mathfrak{m}_{r}}$. Define $\tilde{\lambda}^{\prime} \in \mathbf{C}_{\mathfrak{m}_{r}^{\prime}}$ similarly. Clearly, for any $(i, j) \in X_{\mathfrak{m}_{r}, \mathfrak{m}_{r}^{\prime}}$, the $Y_{\mathfrak{m}_{r}, \mathfrak{m}_{r}^{\prime}}$-coordinates of $v_{i, j}^{\mathfrak{m}, \mathfrak{m}^{\prime}}\left(\lambda, \lambda^{\prime}\right)$ coincide with those of $v_{i, j}^{\mathfrak{m}_{r}, \mathfrak{m}_{r}^{\prime}}\left(\tilde{\lambda}, \tilde{\lambda}^{\prime}\right)$. On the other hand, the assumptions (20a) and (20b) imply that the coordinates outside $Y_{\mathfrak{m}_{r}, \mathfrak{m}_{r}^{\prime}}$ vanish. This clearly implies the third part of Proposition 5.

The last part of Proposition 5 is proved similarly.

Corollary 4 is now an immediate consequence. 


\subsection{Proof of first part of Proposition 4}

For any $0 \neq \mathfrak{m}=\sum_{i \in I} \Delta_{i} \in \mathbb{N}(\Sigma)$ we write $\mathfrak{m}=\mathfrak{m}_{\mathrm{mx}}+\mathfrak{m}_{\mathrm{nmx}}$ where $\mathfrak{m}_{\mathrm{mx}}=$ $\sum_{i \in I: e}\left(\Delta_{i}\right)=\max \mathfrak{m} \Delta_{i}$ and $\mathfrak{m}_{\mathrm{nmx}}=\sum_{i \in I: e\left(\Delta_{i}\right)<\max \mathfrak{m}} \Delta_{i}$. For consistency we also write $0_{\mathrm{mx}}=0_{\mathrm{nmx}}=0$.

Lemma 8 For any $\mathfrak{m} \in \mathbb{N}(\Sigma)$, the representation $L\left(\mathfrak{m}_{\mathrm{nmx}}\right) \times L\left(\mathfrak{m}_{\mathrm{mx}}\right)$ is $S I$ and we have $L(\mathfrak{m})=\operatorname{soc}\left(L\left(\mathfrak{m}_{\mathrm{nmx}}\right) \times L\left(\mathfrak{m}_{\mathrm{mx}}\right)\right)$. Moreover, suppose that $L\left(\mathfrak{m}^{\prime}\right)$ is an irreducible subquotient of $L\left(\mathfrak{m}_{\mathrm{nmx}}\right) \times L\left(\mathfrak{m}_{\mathrm{mx}}\right)$ such that $\mathfrak{m}_{\mathrm{mx}}^{\prime}=\mathfrak{m}_{\mathrm{mx}}$. Then, $\mathfrak{m}^{\prime}=\mathfrak{m}$.

This follows from (10) and Lemmas 5 and 7.

Lemma 9 Suppose that $1 \neq \pi \in \operatorname{Irr}$ and $0 \neq \mathfrak{m} \in \mathbb{N}(\Sigma)$ with $\max \operatorname{supp} \pi<$ $\max \mathfrak{m}$. Then

$$
\begin{aligned}
& \operatorname{soc}(\pi \times L(\mathfrak{m}))=\operatorname{soc}\left(\pi \times \operatorname{soc}\left(L\left(\mathfrak{m}_{\mathrm{nmx}}\right) \times L\left(\mathfrak{m}_{\mathrm{mx}}\right)\right)\right) \\
& \quad=\operatorname{soc}\left(\operatorname{soc}\left(\pi \times L\left(\mathfrak{m}_{\mathrm{nmx}}\right)\right) \times L\left(\mathfrak{m}_{\mathrm{mx}}\right)\right)=\operatorname{soc}\left(\pi \times L\left(\mathfrak{m}_{\mathrm{nmx}}\right) \times L\left(\mathfrak{m}_{\mathrm{mx}}\right)\right) .
\end{aligned}
$$

In particular, for any $\mathfrak{m}^{\prime} \in \mathbb{N}(\Sigma)$

$$
L\left(\mathfrak{m}^{\prime}\right) \hookrightarrow \pi \times L(\mathfrak{m}) \Longleftrightarrow \mathfrak{m}_{\mathrm{mx}}^{\prime}=\mathfrak{m}_{\mathrm{mx}} \text { and } L\left(\mathfrak{m}_{\mathrm{nmx}}^{\prime}\right) \hookrightarrow \pi \times L\left(\mathfrak{m}_{\mathrm{nmx}}\right)
$$

Proof The first equality of (28) follows from Lemma 8 while the last one follows from Lemma 6 , Corollary 6 and the condition on $\pi$. It remains to show that any irreducible subrepresentation $\pi^{\prime}$ of $\pi \times L\left(\mathfrak{m}_{\mathrm{nmx}}\right) \times L\left(\mathfrak{m}_{\mathrm{mx}}\right)$ is contained in $\pi \times L(\mathfrak{m})$. Assume on the contrary that this is not the case. Then $\pi^{\prime} \hookrightarrow \pi \times L\left(\mathfrak{m}^{\prime \prime}\right)$ for some $L\left(\mathfrak{m}^{\prime \prime}\right) \leq L\left(\mathfrak{m}_{\mathrm{nmx}}\right) \times L\left(\mathfrak{m}_{\mathrm{mx}}\right)$ with $\mathfrak{m}_{\mathrm{mx}}^{\prime \prime} \neq \mathfrak{m}_{\mathrm{mx}}$ (by the second part of Lemma 8). On the other hand, by the last equality of (28) we have $\pi^{\prime} \hookrightarrow \tau \times L\left(\mathfrak{m}_{\mathrm{mx}}\right)$ for some irreducible $\tau \hookrightarrow \pi \times L\left(\mathfrak{m}_{\mathrm{nmx}}\right)$ and hence by Lemma 8 we have $\mathfrak{m}_{\mathrm{mx}}^{\prime}=\mathfrak{m}_{\mathrm{mx}}$ where we write $\pi^{\prime}=L\left(\mathfrak{m}^{\prime}\right)$.

Now, $\pi^{\prime} \hookrightarrow \pi \times L\left(\mathfrak{m}_{\mathrm{nmx}}^{\prime \prime}\right) \times L\left(\mathfrak{m}_{\mathrm{mx}}^{\prime \prime}\right)$. Hence, $\pi^{\prime} \hookrightarrow \tau^{\prime} \times L\left(\mathfrak{m}_{\mathrm{mx}}^{\prime \prime}\right)$ for some irreducible subquotient $\tau^{\prime}$ of $\pi \times L\left(\mathfrak{m}_{\mathrm{nmx}}^{\prime \prime}\right)$. It follows from Lemma 8 that $\mathfrak{m}_{\mathrm{mx}}^{\prime \prime}=\mathfrak{m}_{\mathrm{mx}}^{\prime}=\mathfrak{m}_{\mathrm{mx}}$. We get a contradiction.

The last part follows from (28) and Lemma 8.

Corollary 7 Let $\pi$ and $\mathfrak{m}$ be as in Lemma 9. Let $\Delta$ be a segment in $\mathfrak{m}$ such that $e(\Delta)=\max \mathfrak{m}$. Then, for any $\mathfrak{m}^{\prime} \in \mathbb{N}(\Sigma)$

$$
\begin{aligned}
L\left(\mathfrak{m}^{\prime}\right) \hookrightarrow \pi \times L(\mathfrak{m}) \Longleftrightarrow & \Delta \text { is contained in } \mathfrak{m}^{\prime} \text { and } \\
& L\left(\mathfrak{m}^{\prime}-\Delta\right) \hookrightarrow \pi \times L(\mathfrak{m}-\Delta) .
\end{aligned}
$$


Indeed, if $\max (\mathfrak{m}-\Delta) \neq \max \mathfrak{m}$, i.e., if $\mathfrak{m}_{\mathrm{mx}}=\Delta$ then this is just Lemma 9; otherwise this follows from Lemma 9 by applying it to both $\mathfrak{m}$ and $\mathfrak{m}-\Delta$ (with $\mathfrak{m}^{\prime}$ and $\mathfrak{m}^{\prime}-\Delta$ respectively).

In fact, the following slightly more precise statement holds (although we shall not use it here).

Corollary 8 Under the conditions of Corollary 7 we have

$$
\begin{aligned}
\operatorname{soc}(\pi \times L(\mathfrak{m})) & =\operatorname{soc}(\operatorname{soc}(\pi \times L(\mathfrak{m}-\Delta)) \times L(\Delta)) \\
& =\operatorname{soc}(\pi \times L(\mathfrak{m}-\Delta) \times L(\Delta)) .
\end{aligned}
$$

Proof Since

$$
\begin{aligned}
& \pi \times L(\mathfrak{m}) \hookrightarrow \pi \times L(\mathfrak{m}-\Delta) \times L(\Delta) \hookrightarrow \pi \times L\left(\mathfrak{m}_{\mathrm{nmx}}\right) \times L\left(\mathfrak{m}_{\mathrm{mx}}-\Delta\right) \\
& \quad \times L(\Delta)
\end{aligned}
$$

and $L\left(\mathfrak{m}_{\mathrm{mx}}\right)=L\left(\mathfrak{m}_{\mathrm{mx}}-\Delta\right) \times L(\Delta)$, the equality $\operatorname{soc}(\pi \times L(\mathfrak{m}))=\operatorname{soc}(\pi \times$ $L(\mathfrak{m}-\Delta) \times L(\Delta))$ follows from Lemma 9 which also implies that

$$
\operatorname{soc}(\pi \times L(\mathfrak{m}))=\operatorname{soc}\left(\operatorname{soc}\left(\pi \times L\left(\mathfrak{m}_{\mathrm{nmx}}\right)\right) \times L\left(\mathfrak{m}_{\mathrm{mx}}-\Delta\right) \times L(\Delta)\right) .
$$

Note that since $L\left(\mathfrak{m}_{\mathrm{mx}}\right)=L\left(\mathfrak{m}_{\mathrm{mx}}-\Delta\right) \times L(\Delta)$ is $\square$-irreducible, for any $\sigma \in \operatorname{Irr}$ we have

$$
\operatorname{soc}\left(\sigma \times L\left(\mathfrak{m}_{\mathrm{mx}}-\Delta\right) \times L(\Delta)\right)=\operatorname{soc}\left(\operatorname{soc}\left(\sigma \times L\left(\mathfrak{m}_{\mathrm{mx}}-\Delta\right)\right) \times L(\Delta)\right) .
$$

The same is therefore true for any semisimple $\sigma$. Since $\operatorname{soc}\left(\pi \times L\left(\mathfrak{m}_{\mathrm{nmx}}\right)\right)$ is semisimple (by definition of soc) we infer that

$$
\operatorname{soc}(\pi \times L(\mathfrak{m}))=\operatorname{soc}\left(\operatorname{soc}\left(\operatorname{soc}\left(\pi \times L\left(\mathfrak{m}_{\mathrm{nmx}}\right)\right) \times L\left(\mathfrak{m}_{\mathrm{mx}}-\Delta\right)\right) \times L(\Delta)\right) .
$$

On the other hand, applying Lemma 9 to $\mathfrak{m}-\Delta($ if $\max (\mathfrak{m}-\Delta)=\max \mathfrak{m})$ we have

$$
\operatorname{soc}(\pi \times L(\mathfrak{m}-\Delta))=\operatorname{soc}\left(\operatorname{soc}\left(\pi \times L\left(\mathfrak{m}_{\mathrm{nmx}}\right)\right) \times L\left(\mathfrak{m}_{\mathrm{mx}}-\Delta\right)\right) .
$$

(If $\max (\mathfrak{m}-\Delta) \neq \max \mathfrak{m}$ then the last relation is trivial.) Hence,

$$
\operatorname{soc}(\pi \times L(\mathfrak{m}))=\operatorname{soc}(\operatorname{soc}(\pi \times L(\mathfrak{m}-\Delta)) \times L(\Delta)) .
$$

The corollary follows. 
We can now prove the first part of Proposition 4, which is a strengthening of [19, Lemma 4.16].

Suppose first that $\left(\mathfrak{m}+\mathfrak{m}^{\prime}\right)^{-}=\mathfrak{m}+\mathfrak{m}^{\prime-}$. Then, by comparing supp of both sides, $\Delta\left(\mathfrak{m}+\mathfrak{m}^{\prime}\right)=\Delta\left(\mathfrak{m}^{\prime}\right)$. For simplicity write $\Delta=\Delta\left(\mathfrak{m}^{\prime}\right)$ and $\pi=Z(\mathfrak{m})$. By Corollary 7 ,

$$
\begin{aligned}
Z\left(\mathfrak{m}+\mathfrak{m}^{\prime}\right) & =L\left(\left(\mathfrak{m}+\mathfrak{m}^{\prime}\right)^{\#}\right) \leq \operatorname{soc}\left(\pi \times L\left(\mathfrak{m}^{\prime \#}\right)\right)=\operatorname{soc}\left(\pi \times Z\left(\mathfrak{m}^{\prime}\right)\right) \Longleftrightarrow \\
Z\left(\mathfrak{m}+\mathfrak{m}^{\prime-}\right) & =Z\left(\left(\mathfrak{m}+\mathfrak{m}^{\prime}\right)^{-}\right)=L\left(\left(\mathfrak{m}+\mathfrak{m}^{\prime}\right)^{\#}-\Delta\right) \\
& \leq \operatorname{soc}\left(\pi \times L\left(\mathfrak{m}^{\prime \#}-\Delta\right)\right)=\operatorname{soc}\left(\pi \times Z\left(\mathfrak{m}^{\prime-}\right)\right)
\end{aligned}
$$

as required.

It remains therefore to show that $\mathrm{SA}\left(\mathfrak{m}, \mathfrak{m}^{\prime}\right)$ implies $\left(\mathfrak{m}+\mathfrak{m}^{\prime}\right)^{-}=\mathfrak{m}+\mathfrak{m}^{\prime}$. The argument is essentially in [19]. For completeness we recall it. Write $\mathfrak{m}=$ $\sum_{i \in I} \Delta_{i}, \mathfrak{m}^{\prime}=\sum_{i^{\prime} \in I^{\prime}} \Delta_{i^{\prime}}$ and let $i_{1}^{\prime}, \ldots, i_{m^{\prime}}^{\prime}$ be leading indices of $\mathfrak{m}^{\prime}$. Assume on the contrary that $\left(\mathfrak{m}+\mathfrak{m}^{\prime}\right)^{-} \neq \mathfrak{m}+\mathfrak{m}^{\prime-}$. Then, there exist indices $l^{\prime} \leq m^{\prime}$ and $l \in I$ such that $\Delta_{l} \prec \Delta_{i_{l^{\prime}}}, e\left(\Delta_{l}\right)=e\left(\overleftarrow{\Delta}_{i_{l^{\prime}}^{\prime}}\right)$ and either $l^{\prime}=m^{\prime}$ or $\Delta_{l} \subsetneq \Delta_{i_{l^{\prime}+1}^{\prime}}$ By Corollary 4 we may replace $\mathfrak{m}$ and $\mathfrak{m}^{\prime}$ by $\mathfrak{m}_{\geq \Delta_{l}}$ and $\mathfrak{m}_{\geq \Delta_{l}}^{\prime}$ respectively since neither the condition $\mathrm{SA}\left(\mathfrak{m}, \mathfrak{m}^{\prime}\right)$ nor the assumption $\left(\mathfrak{m}+\mathfrak{m}^{\prime}\right)^{-} \neq \mathfrak{m}+\mathfrak{m}^{\prime-}$ is affected by this change. Thus, we may assume without loss of generality that $\mathfrak{m}=\mathfrak{m}_{\geq \Delta_{l}}$ and $l^{\prime}=m^{\prime}$. By Lemma 9 (applied to $\mathfrak{m}^{\prime \prime \#}$ ), the condition $\mathrm{SA}\left(\mathfrak{m}, \mathfrak{m}^{\prime}\right)$ implies that

$$
\mathfrak{m}_{\mathrm{mx}}^{\prime \#}=\left(\mathfrak{m}+\mathfrak{m}^{\prime}\right)_{\mathrm{mx}}^{\#}
$$

However, the right-hand side admits a segment which contains $e\left(\Delta_{l}\right)$ in its support while the left-hand side does not. We obtain a contradiction.

\subsection{Proof of second part of Proposition 4}

Lemma 10 Let $\mathfrak{m}=\sum_{i \in I} \Delta_{i} \in \mathbb{N}(\Sigma)$ and let $i_{1}, \ldots, i_{m}$ be leading indices

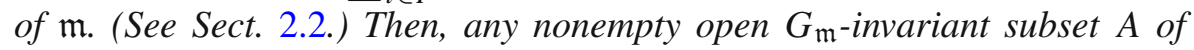
$\mathbf{C}_{\mathfrak{m}}$ contains an element $\lambda$ whose coordinates satisfy $\lambda_{i, i_{j}}=\delta_{i, i_{j+1}}$ for all $j=1, \ldots, m-1$ and $i \in I$ such that $e\left(\Delta_{i}\right)=e\left(\Delta_{i_{j+1}}\right)$.

Proof We argue as in [24]. We prove by induction on $l=0, \ldots, m-1$ that there exists $\lambda \in A$ such $\lambda_{i, i_{j}}=\delta_{i, i_{j+1}}$ for all $j=1, \ldots, l$ and $i \in I$ such that $e\left(\Delta_{i}\right)=e\left(\Delta_{i_{j+1}}\right)$. The base of the induction $(l=0)$ is trivial. For the induction step, we assume that $0<l<m$ and the statement holds for $l-1$. In addition, by openness, we may assume that $\lambda_{i_{l+1}, i_{l}} \neq 0$. Take $g \in G_{\mathfrak{m}}$ whose coordinates are given by 


$$
g_{i, j}= \begin{cases}\lambda_{i, i_{l}} & \text { if } j=i_{l+1} \text { and } e\left(\Delta_{i}\right)=e\left(\Delta_{i_{l+1}}\right), \quad(i, j) \in Y_{\mathfrak{m}} . \\ \delta_{i, j} & \text { otherwise }\end{cases}
$$

( $g$ is invertible since $\lambda_{i_{l+1}, i_{l}} \neq 0$.) Let $\tilde{\lambda}=g^{-1} \lambda g \in A$. We show that $\tilde{\lambda}$ satisfies the required conditions for $l$. Suppose first that $j<l$. By assumption, for any $\rho \in \Delta_{i_{j}}$ we have $\lambda x_{\rho, i_{j}}=x_{\overleftarrow{\rho}, i_{j+1}}+\xi$ where the coordinates of $\xi$ with respect to $x_{\leftarrow, i}$ vanish if $e\left(\Delta_{i}\right)=e\left(\Delta_{i_{j+1}}\right)$. The same condition holds for $g^{-1} \xi$. It follows that

$$
\tilde{\lambda} x_{\rho, i_{j}}=g^{-1} \lambda g x_{\rho, i_{j}}=g^{-1} \lambda x_{\rho, i_{j}}=x_{\stackrel{\leftarrow}{\leftarrow}, i_{j+1}}+g^{-1} \xi
$$

and hence, $\tilde{\lambda}_{i, i_{j}}=\delta_{i, i_{j+1}}$ for all $i$ such that $e\left(\Delta_{r}\right)=e\left(\Delta_{i_{j+1}}\right)$. Also, we may write

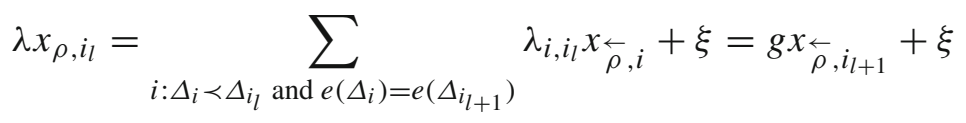

where the coordinates of $\xi$ with respect to $x_{\rho, i}$ vanish if $e\left(\Delta_{i}\right)=e\left(\Delta_{i_{l+1}}\right)$. Hence,

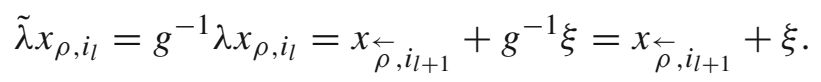

It follows that $\tilde{\lambda}_{i, i_{l}}=\delta_{i, i_{l+1}}$ for all $i$ such that $e\left(\Delta_{i}\right)=e\left(\Delta_{i_{l+1}}\right)$. This concludes the induction step.

Similarly, we have the following.

Lemma 11 Let $\mathfrak{m} \in \mathbb{N}(\Sigma)$. Then, any nonempty open $G_{\mathfrak{m}^{-}}$-invariant subset $B$ of $\mathbf{C}_{\mathfrak{m}^{-}}$contains an element $\lambda$ whose coordinates satisfy $\lambda_{i, j}=0$ for all $(i, j) \in X_{\mathfrak{m}^{-}} \backslash X_{\mathfrak{m}}$.

Proof Let $i_{1}, \ldots, i_{m}$ be leading indices of $\mathfrak{m}$. Note that

$$
X_{\mathfrak{m}^{-}} \backslash X_{\mathfrak{m}}=\left\{\left(i_{j}, i\right) \in X_{\mathfrak{m}^{-}}: j=2, \ldots, m \text { and } e\left(\Delta_{i_{j}}\right)=e\left(\Delta_{i}\right)\right\}
$$

We show by descending induction on $l=m, \ldots, 1$ that there exists $\lambda \in B$ such that $\lambda_{i_{j}, i}=0$ for all $j>l$ and $i$ such that $e\left(\Delta_{i_{j}}\right)=e\left(\Delta_{i}\right)$. Once again, the base of the induction $(l=m)$ is trivial.

For the induction step we may assume that $\Delta_{i_{l-1}}$ is not a singleton (or equivalently, $\left.\left(i_{l}, i_{l-1}\right) \in X_{\mathfrak{m}^{-}}\right)$since otherwise, $\left(i_{l}, i\right) \notin X_{\mathfrak{m}^{-}}$for all $i$ such 
that $e\left(\Delta_{i_{j}}\right)=e\left(\Delta_{i}\right)$. We may also assume that $\lambda \in B$ satisfies $\lambda_{i_{l}, i_{l-1}} \neq 0$. Consider the set

$$
I_{l}=\left\{i \in I:\left(i_{l}, i\right) \in X_{\mathfrak{m}^{-}} \text {and } e\left(\Delta_{i_{l}}\right)=e\left(\Delta_{i}\right)\right\} .
$$

Note that it follows from the definition of $i_{l}$ that $\left(i_{l-1}, i\right) \in Y_{\mathfrak{m}^{-}}$for all $i \in I_{l}$. Let $g \in G_{\mathfrak{m}^{-}}$be such that $g_{i_{l-1}, i}=-\lambda_{i_{l}, i_{l-1}}^{-1} \lambda_{i_{l}, i}$ for all $i \in I_{l}$ and $g_{i, j}=\delta_{i, j}$ if $j \neq I_{l}$ or $i \neq i_{l-1}$. Let $\tilde{\lambda}=g^{-1} \lambda g \in B$. Then, for any $\rho \in \Delta_{i}, i \in I_{l}$ the coefficients of

$$
\lambda g x_{\rho, i}=\lambda\left(x_{\rho, i}-\lambda_{i_{l}, i_{l-1}}^{-1} \lambda_{i_{l}, i} x_{\rho, i_{l-1}}\right)
$$

with respect to $x_{\leftarrow}^{\leftarrow}, j$ are 0 for all $j$ such that $\Delta_{j} \geq \Delta_{i_{l}}$. Hence, the coefficient of $x_{\overleftarrow{\rho}_{,} i_{l}}$ in $\tilde{\lambda} x_{\rho, i}$ is 0 as well. Thus, $\tilde{\lambda}_{i_{l}, i}=0$ for all $i \in I_{l}$. Moreover, if $i \in I_{j}$ with $j>l$ then $\tilde{\lambda} x_{\rho, i}=g^{-1} \lambda x_{\rho, i}=\lambda x_{\rho, i}$ since $g$ fixes $x_{\leftarrow, k}$ for all $k$ such that $\Delta_{k} \leq \Delta_{i_{l}}$. It follows from the induction hypothesis that $\tilde{\lambda}_{i_{j}, i}=\lambda_{i_{j}, i}=0$ for all $i \in I_{j}$. This completes the induction step.

Recall the sets $X_{\mathfrak{m}, \mathfrak{m}^{\prime}}$ and $Y_{\mathfrak{m}, \mathfrak{m}^{\prime}}$ defined in Sect. 5.4.

Lemma 12 Suppose that $0 \neq \mathfrak{m}, \mathfrak{m}^{\prime} \in \mathbb{N}(\Sigma)$ with $\max \mathfrak{m}<\max \mathfrak{m}^{\prime}$. Write $\mathfrak{m}=\sum_{i \in I} \Delta_{i}, \mathfrak{m}^{\prime}=\sum_{i \in I^{\prime}} \Delta_{i^{\prime}}$ and let $i_{1}^{\prime}, \ldots, i_{m^{\prime}}^{\prime}$ be leading indices of $\mathfrak{m}^{\prime}$. Write $\mathfrak{m}^{\prime-}=\sum_{i \in I^{\prime}} \Delta_{i^{\prime}}^{\prime}$ where

$$
\Delta_{i^{\prime}}^{\prime}= \begin{cases}\Delta_{i^{\prime}} \backslash\left\{e\left(\Delta_{i^{\prime}}\right)\right\} & \text { if } i^{\prime}=i_{j^{\prime}}^{\prime} \text { for some } j^{\prime}=1, \ldots, m^{\prime}, \\ \Delta_{i^{\prime}} & \text { otherwise. }\end{cases}
$$

Then, $X_{\mathfrak{m}, \mathfrak{m}^{\prime-}} \subseteq X_{\mathfrak{m}, \mathfrak{m}^{\prime}}$ and $Y_{\mathfrak{m}, \mathfrak{m}^{\prime}} \subseteq Y_{\mathfrak{m}, \mathfrak{m}^{\prime} . \text { Let }} \tilde{X}_{\mathfrak{m}, \mathfrak{m}^{\prime}}=X_{\mathfrak{m}, \mathfrak{m}^{\prime}} \backslash X_{\mathfrak{m}, \mathfrak{m}^{\prime-}}$ and $\tilde{Y}_{\mathfrak{m}, \mathfrak{m}^{\prime}}=Y_{\mathfrak{m}, \mathfrak{m}^{\prime}} \backslash Y_{\mathfrak{m}, \mathfrak{m}^{\prime}}$ and let $f_{\mathfrak{m}, \mathfrak{m}^{\prime}}: \tilde{Y}_{\mathfrak{m}, \mathfrak{m}^{\prime}} \rightarrow \tilde{X}_{\mathfrak{m}, \mathfrak{m}^{\prime}}$ be given by $\left(i, i_{j^{\prime}}^{\prime}\right) \mapsto\left(i, i_{j^{\prime}-1}^{\prime}\right)$. Then, upon fixing a total order on $I$, the function $f_{\mathfrak{m}, \mathfrak{m}^{\prime}}$ is strictly monotone increasing with respect to the lexicographic order, and in particular injective. Moreover, $f_{\mathfrak{m}, \mathfrak{m}^{\prime}}$ is a bijection (i.e., onto) if and only if $\left(\mathfrak{m}+\mathfrak{m}^{\prime}\right)^{-}=\mathfrak{m}+\mathfrak{m}^{\prime-}$. In particular, this is the case if $\# \tilde{X}_{\mathfrak{m}, \mathfrak{m}^{\prime}} \leq \# \tilde{Y}_{\mathfrak{m}, \mathfrak{m}^{\prime}}$.

Proof The fact that $X_{\mathfrak{m}, \mathfrak{m}^{\prime}} \subseteq X_{\mathfrak{m}, \mathfrak{m}^{\prime}}$ and $Y_{\mathfrak{m}, \mathfrak{m}^{\prime}} \subseteq Y_{\mathfrak{m}, \mathfrak{m}^{\prime}}$ is clear. The function $f_{\mathfrak{m}, \mathfrak{m}^{\prime}}$ is well-defined since $\max \mathfrak{m}<\max \mathfrak{m}^{\prime}$. Note that

$$
\tilde{X}_{\mathfrak{m}, \mathfrak{m}^{\prime}}=\left\{\left(i, i_{j^{\prime}}^{\prime}\right) \in X_{\mathfrak{m}, \mathfrak{m}^{\prime}}: j^{\prime}=1, \ldots, m^{\prime}, e\left(\Delta_{i}\right)=e\left(\overleftarrow{\Delta}_{i_{j^{\prime}}^{\prime}}\right)\right\}
$$

and

$$
\tilde{Y}_{\mathfrak{m}, \mathfrak{m}^{\prime}}=\left\{\left(i, i_{j^{\prime}}^{\prime}\right) \in Y_{\mathfrak{m}, \mathfrak{m}^{\prime}}: j^{\prime}=2, \ldots, m^{\prime}, e\left(\Delta_{i}\right)=e\left(\Delta_{i_{j^{\prime}}^{\prime}}\right)\right\}
$$


The monotonicity of $f_{\mathfrak{m}, \mathfrak{m}^{\prime}}$ is obvious. The equivalence of $\left(\mathfrak{m}+\mathfrak{m}^{\prime}\right)^{-}=\mathfrak{m}+$ $\mathfrak{m}^{\prime-}$ and the surjectivity of $f_{\mathfrak{m}, \mathfrak{m}^{\prime}}$ is easy to check (cf., proof of [19, Proposition 6.19]).

We are now ready to show the second part of Proposition 4, let $i_{1}^{\prime}, \ldots, i_{m^{\prime}}^{\prime}$ be leading indices of $\mathfrak{m}^{\prime}$.

As in Lemma 12 we view $X_{\mathfrak{m}, \mathfrak{m}^{\prime}}$ and $Y_{\mathfrak{m}, \mathfrak{m}^{\prime-}}$ as subsets of $X_{\mathfrak{m}, \mathfrak{m}^{\prime}}$ and $Y_{\mathfrak{m}, \mathfrak{m}^{\prime}}$ respectively and let $\tilde{X}_{\mathfrak{m}, \mathfrak{m}^{\prime}}=X_{\mathfrak{m}, \mathfrak{m}^{\prime}} \backslash X_{\mathfrak{m}, \mathfrak{m}^{\prime}}$ and $\tilde{Y}_{\mathfrak{m}, \mathfrak{m}^{\prime}}=Y_{\mathfrak{m}, \mathfrak{m}^{\prime}} \backslash Y_{\mathfrak{m}, \mathfrak{m}^{\prime}}$.

Assume first that $\mathrm{SG}\left(\mathfrak{m}, \mathfrak{m}^{\prime}\right)$ holds. Thus, $v_{i, j}^{\mathfrak{m}, \mathfrak{m}^{\prime}}\left(\lambda, \lambda^{\prime}\right),(i, j) \in X_{\mathfrak{m}, \mathfrak{m}^{\prime}}$ are linearly independent in $\mathbb{C}^{Y_{\mathfrak{m}, \mathfrak{m}^{\prime}}}$ for some $\left(\lambda, \lambda^{\prime}\right) \in \mathbf{C}_{\mathfrak{m}} \times \mathbf{C}_{\mathfrak{m}^{\prime}}$ (see (17)). Let

$$
\begin{aligned}
& \tilde{X}_{\mathfrak{m}^{\prime}}=\left\{\left(i^{\prime}, i_{j^{\prime}}^{\prime}\right) \in X_{\mathfrak{m}^{\prime}}: j^{\prime}=1, \ldots, m^{\prime}-1, i^{\prime} \neq i_{j^{\prime}+1}^{\prime} \text { and } e\left(\Delta_{i^{\prime}}\right)=e\left(\Delta_{i_{j^{\prime}+1}^{\prime}}\right)\right\} \\
& \hat{X}_{\mathfrak{m}^{\prime}}=\left\{\left(i_{j^{\prime}}^{\prime}, i^{\prime}\right) \in X_{\mathfrak{m}^{\prime}}: e\left(\Delta_{i_{j^{\prime}}}\right)=b\left(\overleftarrow{\Delta}_{i^{\prime}}\right)\right\}
\end{aligned}
$$

Note that $X_{\mathfrak{m}^{\prime}} \backslash X_{\mathfrak{m}^{\prime-}}=\tilde{X}_{\mathfrak{m}^{\prime}} \cup \hat{X}_{\mathfrak{m}^{\prime}}$. By Lemma 10 (applied to $\mathfrak{m}^{\prime}$ ) we can assume without loss of generality that $\lambda_{i, j}^{\prime}=0$ for all $(i, j) \in \tilde{X}_{\mathfrak{m}^{\prime}}$.

Let pr: $\mathbb{C}^{Y_{\mathfrak{m}, \mathfrak{m}^{\prime}}} \rightarrow \mathbb{C}^{Y_{\mathfrak{m}, \mathfrak{m}^{\prime-}}}$ be the standard projection. It is easy to check that the vanishing of $\lambda_{i, j}^{\prime}$ for $(i, j) \in \tilde{X}_{\mathfrak{m}^{\prime}}$ (regardless of the values of $\lambda_{i, j}^{\prime}$, $\left.(i, j) \in \hat{X}_{\mathfrak{m}^{\prime}}\right)$ implies that $\operatorname{pr}\left(v_{i, j}^{\mathfrak{m}, \mathfrak{m}^{\prime}}\left(\lambda, \lambda^{\prime}\right)\right)=0$ for all $(i, j) \in \tilde{X}_{\mathfrak{m}, \mathfrak{m}^{\prime}}$. In particular, \#䄼, $\mathfrak{m}^{\prime} \leq \# \tilde{Y}_{\mathfrak{m}, \mathfrak{m}^{\prime}}$. By Lemma 12 we infer that $\left(\mathfrak{m}+\mathfrak{m}^{\prime}\right)^{-}=\mathfrak{m}+\mathfrak{m}^{\prime-}$ and $\# \tilde{X}_{\mathfrak{m}, \mathfrak{m}^{\prime}}=\# \tilde{Y}_{\mathfrak{m}, \mathfrak{m}^{\prime}}$. It also follows that $\operatorname{pr}\left(v_{i, j}^{\mathfrak{m}, \mathfrak{m}^{\prime}}\left(\lambda, \lambda^{\prime}\right)\right),(i, j) \in X_{\mathfrak{m}, \mathfrak{m}^{\prime-}}$ are linearly independent in $\mathbb{C}^{Y_{\mathfrak{m}, \mathfrak{m}^{\prime}-}}$. However, once again because of the condition on $\lambda^{\prime}$, we have $\operatorname{pr}\left(v_{i, j}^{\mathfrak{m}, \mathfrak{m}^{\prime}}\left(\lambda, \lambda^{\prime}\right)\right)=v_{i, j}^{\mathfrak{m}, \mathfrak{m}^{\prime-}}\left(\lambda, \tilde{\lambda}^{\prime}\right)$ for all $(i, j) \in X_{\mathfrak{m}, \mathfrak{m}^{\prime}}$ where the coefficients of $\tilde{\lambda}^{\prime} \in \mathbf{C}_{\mathfrak{m}^{\prime-}}$ are given by $\tilde{\lambda}_{i, j}^{\prime}=\lambda_{i, j}^{\prime}$ if $(i, j) \in X_{\mathfrak{m}^{\prime}} \cap X_{\mathfrak{m}^{\prime}}$ and $\tilde{\lambda}_{i, j}^{\prime}=0$ if $(i, j) \in X_{\mathfrak{m}^{\prime}} \backslash X_{\mathfrak{m}^{\prime}}$. Hence, $\mathrm{SG}\left(\mathfrak{m}, \mathfrak{m}^{\prime-}\right)$ holds.

Conversely, assume that $\mathrm{SG}\left(\mathfrak{m}, \mathfrak{m}^{\prime-}\right)$ and $\left(\mathfrak{m}+\mathfrak{m}^{\prime}\right)^{-}=\mathfrak{m}+\mathfrak{m}^{\prime-}$. Let $\left(\lambda, \tilde{\lambda}^{\prime}\right) \in \mathbf{C}_{\mathfrak{m}} \times \mathbf{C}_{\mathfrak{m}^{\prime}}$ be such that $v_{i, j}^{\mathfrak{m}, \mathfrak{m}^{-}}\left(\lambda, \tilde{\lambda}^{\prime}\right),(i, j) \in X_{\mathfrak{m}, \mathfrak{m}^{\prime}}$ are linearly independent in $\mathbb{C}^{Y_{\mathfrak{m}, \mathfrak{m}^{\prime}}}$, and let $f=f_{\mathfrak{m}, \mathfrak{m}^{\prime}}: \tilde{Y}_{\mathfrak{m}, \mathfrak{m}^{\prime}} \rightarrow \tilde{X}_{\mathfrak{m}, \mathfrak{m}^{\prime}}$ be the bijection defined in Lemma 12. By Lemma 11 we may assume without loss of generality that $\tilde{\lambda}_{i, j}^{\prime}=0$ if $(i, j) \in X_{\mathfrak{m}^{\prime}-} \backslash X_{\mathfrak{m}^{\prime}}$. We may also assume that $\tilde{\lambda}_{i_{j^{\prime}+1}^{\prime}, i_{j^{\prime}}^{\prime}}^{\prime} \neq 0$ for all $j^{\prime}=1, \ldots, m^{\prime}-1$ such that $\Delta_{i_{j^{\prime}}^{\prime}}$ is not a singleton. Define $\lambda^{\prime} \in \mathbf{C}_{\mathfrak{m}^{\prime}}$ by

$$
\lambda_{i, j}^{\prime}= \begin{cases}\tilde{\lambda}_{i, j}^{\prime} & (i, j) \in X_{\mathfrak{m}^{\prime}} \cap X_{\mathfrak{m}^{\prime-}}, \\ 0 & (i, j) \in \tilde{X}_{\mathfrak{m}^{\prime}}, \\ 1 & (i, j) \in \hat{X}_{\mathfrak{m}^{\prime}} .\end{cases}
$$


As before,

$$
\operatorname{pr}\left(v_{i, j}^{\mathfrak{m}, \mathfrak{m}^{\prime}}\left(\lambda, \lambda^{\prime}\right)\right)= \begin{cases}v_{i, j}^{\mathfrak{m}, \mathfrak{m}^{\prime-}}\left(\lambda, \tilde{\lambda}^{\prime}\right) & (i, j) \in X_{\mathfrak{m}, \mathfrak{m}^{\prime-}} \\ 0 & (i, j) \in \tilde{X}_{\mathfrak{m}, \mathfrak{m}^{\prime}}\end{cases}
$$

To conclude $\operatorname{SG}\left(\mathfrak{m}, \mathfrak{m}^{\prime}\right)$ it suffices to show that $v_{i, j}^{\mathfrak{m}, \mathfrak{m}^{\prime}}\left(\lambda, \lambda^{\prime}\right),(i, j) \in \tilde{X}_{\mathfrak{m}, \mathfrak{m}^{\prime}}$ are linearly independent. Indeed, for any $(i, j) \in \tilde{Y}_{\mathfrak{m}, \mathfrak{m}^{\prime}}$ the $(i, j)$-th coefficient of $v_{f(i, j)}^{\mathfrak{m}, \mathfrak{m}^{\prime}}\left(\lambda, \lambda^{\prime}\right)$ is $\lambda_{j, j^{\prime}}^{\prime} \neq 0$ where $f(i, j)=\left(i, j^{\prime}\right)$ while the only other non-zero coefficients of $v_{i, j}^{\mathfrak{m}, \mathfrak{m}^{\prime}}\left(\lambda, \lambda^{\prime}\right)(i, j) \in \tilde{X}_{\mathfrak{m}, \mathfrak{m}^{\prime}}$ can occur at coordinates $(r, j)$ with $r<i$. Thus, upon ordering $\tilde{X}_{\mathfrak{m}, \mathfrak{m}^{\prime}}$ and $\tilde{Y}_{\mathfrak{m}, \mathfrak{m}^{\prime}}$ lexicographically (with respect to a prescribed total order on $I$ ), the square matrix formed by the $\tilde{Y}_{\mathfrak{m}, \mathfrak{m}^{\prime}}$-coordinates of $v_{i, j}^{\mathfrak{m}, \mathfrak{m}^{\prime}}\left(\lambda, \lambda^{\prime}\right),(i, j) \in \tilde{X}_{\mathfrak{m}, \mathfrak{m}^{\prime}}$ is upper triangular with non-zero diagonal entries.

To complete the proof of Proposition 4 (the very last part), it remains to invoke the penultimate part of Corollary 4.

\subsection{Proof of first part of Theorem 1}

Suppose that $\mathfrak{m}=\Delta_{1}+\cdots+\Delta_{k}$ with $\Delta_{i+1} \prec \Delta_{i}$ for all $i=1, \ldots, k-1$. We show by induction on $k$ that the conditions $\operatorname{SA}\left(\mathfrak{m}, \mathfrak{m}^{\prime}\right)$ and $\operatorname{SG}\left(\mathfrak{m}, \mathfrak{m}^{\prime}\right)$ are equivalent for any multisegment $\mathfrak{m}^{\prime}=\sum_{i \in I^{\prime}} \Delta_{i^{\prime}}$. (The equivalence of the symmetric conditions $\mathrm{SA}\left(\mathfrak{m}^{\prime}, \mathfrak{m}\right)$ and $\operatorname{SG}\left(\mathfrak{m}^{\prime}, \mathfrak{m}\right)$ would then follow from (9) and Remark 3 part 4.) The case $k=0$ is trivial. Assume that $k>0$ and the statement holds for $k-1$. By Proposition 4 we may assume that $\max \mathfrak{m}^{\prime} \leq \max \mathfrak{m}$. In this case, by [19, Proposition 6.1] $\mathrm{SA}\left(\mathfrak{m}, \mathfrak{m}^{\prime}\right)$ is equivalent to $\operatorname{SA}\left(\mathfrak{m}-\Delta_{1}, \mathfrak{m}_{<\Delta_{1}}^{\prime}\right)$. To complete the induction step we show that the conditions $\operatorname{SG}\left(\mathfrak{m}, \mathfrak{m}^{\prime}\right)$ and $\operatorname{SG}\left(\mathfrak{m}-\Delta_{1}, \mathfrak{m}_{<\Delta_{1}}^{\prime}\right)$ are also equivalent.

Note that $X_{\mathfrak{m}, \mathfrak{m}^{\prime}}=X_{\mathfrak{m}-\Delta_{1}, \mathfrak{m}^{\prime} \Delta_{1}} \cup X_{\mathfrak{m}-\Delta_{1}, \mathfrak{m}_{\geq \Delta_{1}}^{\prime}}, Y_{\mathfrak{m}, \mathfrak{m}^{\prime}}=Y_{\mathfrak{m}-\Delta_{1}, \mathfrak{m}^{\prime}} \cup$ $Y_{\Delta_{1}, \mathfrak{m}_{\geq \Delta_{1}}^{\prime}} \cup Y_{\mathfrak{m}-\Delta_{1}, \mathfrak{m}_{\geq \Delta_{1}}^{\prime}}$ and $X_{\mathfrak{m}^{\prime}}=X_{\mathfrak{m}_{<\Delta_{1}}^{\prime}} \cup X_{\mathfrak{m}_{<\Delta_{1}}^{\prime}, \mathfrak{m}_{\geq \Delta_{1}}^{\prime}}$.

We compare the vectors $v_{i, j}^{\mathfrak{m}, \mathfrak{m}^{\prime}}\left(\lambda, \lambda^{\prime}\right) \in \mathbb{C}^{Y_{\mathfrak{m}, \mathfrak{m}^{\prime}}},(i, j) \in X_{\mathfrak{m}, \mathfrak{m}^{\prime}}$ for $\left(\lambda, \lambda^{\prime}\right) \in$

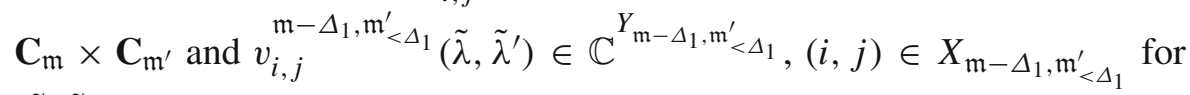
$\left(\tilde{\lambda}, \tilde{\lambda}^{\prime}\right) \in \mathbf{C}_{\mathfrak{m}-\Delta_{1}} \times \mathbf{C}_{\mathfrak{m}_{<\Delta_{1}}^{\prime}}$.

Suppose that $\lambda_{i, j}=\tilde{\lambda}_{i, j}$ for all $(i, j) \in X_{\mathfrak{m}-\Delta_{1}}$ and $\lambda_{i, j}^{\prime}=\tilde{\lambda}_{i, j}^{\prime}$ for all $(i, j) \in X_{\mathfrak{m}_{<\Delta_{1}}^{\prime}}$. Then, it is easy to check that for all $(i, j) \in X_{\mathfrak{m}-\Delta_{1}, \mathfrak{m}_{<\Delta_{1}}^{\prime}}$, the $Y_{\mathfrak{m}-\Delta_{1}, \mathfrak{m}^{\prime} \Delta_{1}}$-coordinates of $v_{i, j}^{\mathfrak{m}, \mathfrak{m}^{\prime}}\left(\lambda, \lambda^{\prime}\right)$ and $v_{i, j}^{\mathfrak{m}-\Delta_{1}, \mathfrak{m}_{<\Delta_{1}}^{\prime}}\left(\tilde{\lambda}, \tilde{\lambda}^{\prime}\right)$ coincide, while the coordinates of $v_{i, j}^{\mathfrak{m}, \mathfrak{m}^{\prime}}\left(\lambda, \lambda^{\prime}\right)$ outside $Y_{\mathfrak{m}-\Delta_{1}, \mathfrak{m}_{<\Delta_{1}}^{\prime}}$ all vanish. It follows 
that $\mathrm{SG}\left(\mathfrak{m}, \mathfrak{m}^{\prime}\right) \Longrightarrow \mathrm{SG}\left(\mathfrak{m}-\Delta_{1}, \mathfrak{m}_{<\Delta_{1}}\right)$. For the converse implication, observe that if $(i, j) \in X_{\mathfrak{m}-\Delta_{1}, \mathfrak{m}_{\geq \Delta_{1}}^{\prime}}$, then $(i-1, j) \in Y_{\mathfrak{m}, \mathfrak{m}_{\geq \Delta_{1}}^{\prime}},(i, i-1) \in X_{\mathfrak{m}}$ and the $(i-1, j)$-coordinate of vectors $v_{i, j}^{\mathfrak{m}, \mathfrak{m}^{\prime}}\left(\lambda, \lambda^{\prime}\right)$ is $\lambda_{i, i-1}$. Moreover, if the $\left(i^{\prime}, j^{\prime}\right)$-coordinate of $v_{i, j}^{\mathfrak{m}, \mathfrak{m}^{\prime}}\left(\lambda, \lambda^{\prime}\right)$ is nonzero for some $\left(i^{\prime}, j^{\prime}\right) \in Y_{\mathfrak{m}, \mathfrak{m}_{\geq \Delta_{1}}^{\prime}}$, then $j^{\prime}=j$ and $i^{\prime}<i$. By imposing the open condition $\lambda_{i, i-1} \neq 0$ for all $(i, i-1) \in X_{\mathfrak{m}}$ we see that $\mathrm{SG}\left(\mathfrak{m}-\Delta_{1}, \mathfrak{m}_{<\Delta_{1}}\right) \Longrightarrow \mathrm{SG}\left(\mathfrak{m}, \mathfrak{m}^{\prime}\right)$. This completes the induction step.

Remark 8 In [19, Proposition 6.15] we also proved that if at least one of $\mathfrak{m}$ and $\mathfrak{m}^{\prime}$ is a ladder then $\operatorname{SG}\left(\mathfrak{m}, \mathfrak{m}^{\prime}\right)$ is equivalent to the condition that there exists an injective map $f: X_{\mathfrak{m}, \mathfrak{m}^{\prime}} \rightarrow Y_{\mathfrak{m}, \mathfrak{m}^{\prime}}$ such that for all $\left(i, i^{\prime}\right) \in X_{\mathfrak{m}, \mathfrak{m}^{\prime}}$ either $f\left(i, i^{\prime}\right)=\left(i, j^{\prime}\right)$ with $\Delta_{j^{\prime}} \prec \Delta_{i^{\prime}}$ or $f\left(i, i^{\prime}\right)=(i, j)$ with $\Delta_{i} \prec \Delta_{j}$. In general, the latter condition is strictly weaker than $\mathrm{SG}\left(\mathfrak{m}, \mathfrak{m}^{\prime}\right)$.

\subsection{Proof of Proposition 6 and second part of Theorem 1}

Suppose that $Z\left(\mathfrak{m}+\mathfrak{m}^{\prime}+\mathfrak{n}\right) \hookrightarrow Z\left(\mathfrak{m}+\mathfrak{m}^{\prime}\right) \times Z(\mathfrak{n})$ and $Z\left(\mathfrak{m}+\mathfrak{m}^{\prime}\right) \hookrightarrow$ $Z(\mathfrak{m}) \times Z\left(\mathfrak{m}^{\prime}\right)$. Then, $Z\left(\mathfrak{m}+\mathfrak{m}^{\prime}+\mathfrak{n}\right) \hookrightarrow Z(\mathfrak{m}) \times Z\left(\mathfrak{m}^{\prime}\right) \times Z(\mathfrak{n})$. Thus, there exists an irreducible subquotient $\pi$ of $Z\left(\mathfrak{m}^{\prime}\right) \times Z(\mathfrak{n})$ such that $Z(\mathfrak{m}+$ $\left.\mathfrak{m}^{\prime}+\mathfrak{n}\right) \hookrightarrow Z(\mathfrak{m}) \times \pi$. Since $Z\left(\mathfrak{m}+\mathfrak{m}^{\prime}+\mathfrak{n}\right) \unlhd Z(\mathfrak{m}) \times Z\left(\mathfrak{m}^{\prime}\right) \times Z(\mathfrak{n})$ and $Z\left(\mathfrak{m}+\mathfrak{m}^{\prime}+\mathfrak{n}\right) \unlhd Z(\mathfrak{m}) \times Z\left(\mathfrak{m}^{\prime}+\mathfrak{n}\right)$, we infer that necessarily $\pi=Z\left(\mathfrak{m}^{\prime}+\mathfrak{n}\right)$. Hence, $\operatorname{SA}\left(\mathfrak{m}, \mathfrak{m}^{\prime}+\mathfrak{n}\right)$.

Now suppose that $\operatorname{SG}\left(\mathfrak{m}+\mathfrak{m}^{\prime}, \mathfrak{n}\right)$ holds. As before, we use the criterion 3 of Proposition 3. Let $\lambda \in \mathbf{C}_{\mathfrak{m}+\mathfrak{m}^{\prime}}$ and $\lambda^{\prime} \in \mathbf{C}_{\mathfrak{n}}$ be such that $v_{i, j}^{\mathfrak{m}+\mathfrak{m}^{\prime}, \mathfrak{n}}\left(\lambda, \lambda^{\prime}\right),(i, j) \in$ $X_{\mathfrak{m}+\mathfrak{m}^{\prime}, \mathfrak{n}}$ are linearly independent in $\mathbb{C}^{Y_{\mathfrak{m}+\mathfrak{m}^{\prime}, \mathfrak{n}}}$. Denote by $\lambda_{\mathfrak{m}}$ the element of $\mathbf{C}_{\mathfrak{m}}$ whose coordinates coincide with the $X_{\mathfrak{m}}$-coordinates of $\lambda$. Similarly for $\lambda_{\mathfrak{m}^{\prime}}$. If $\mathrm{SG}\left(\mathfrak{m}, \mathfrak{m}^{\prime}\right)$ holds, then by Proposition 3 , we may assume that $\lambda_{i, j}=0$ for all $(i, j) \in X_{\mathfrak{m}, \mathfrak{m}^{\prime}}$. (We may still assume that $\left(\lambda_{\mathfrak{m}}, \lambda_{\mathfrak{m}^{\prime}}\right) \in \mathbf{C}_{\mathfrak{m}} \times \mathbf{C}_{\mathfrak{m}^{\prime}}$ is generic.) In this case, the $Y_{\mathfrak{m}^{\prime}, \mathfrak{n}}$-coordinates of $v_{i, j}^{\mathfrak{m}+\mathfrak{m}^{\prime}, \mathfrak{n}}\left(\lambda, \lambda^{\prime}\right),(i, j) \in X_{\mathfrak{m}, \mathfrak{n}}$ vanish. On the other hand, the $Y_{\mathfrak{m}, \mathfrak{n}}$-coordinates of $v_{i, j}^{\mathfrak{m}+\mathfrak{m}^{\prime}, \mathfrak{n}}\left(\lambda, \lambda^{\prime}\right),(i, j) \in X_{\mathfrak{m}, \mathfrak{n}}$ coincide with those of $v_{i, j}^{\mathfrak{m}, \mathfrak{n}}\left(\lambda_{\mathfrak{m}}, \lambda^{\prime}\right)$. The condition $\operatorname{SG}(\mathfrak{m}, \mathfrak{n})$ follows.

Consider $v_{i, j}^{\mathfrak{m}, \mathfrak{m}^{\prime}+\mathfrak{n}}\left(\lambda_{\mathfrak{m}}, \mu\right)$ where $\mu_{\mathfrak{m}^{\prime}, \mathfrak{n}}=\mu_{\mathfrak{n}, \mathfrak{m}^{\prime}}=0$ and $\mu_{\mathfrak{n}}=\lambda^{\prime}$. Then, for $(i, j) \in X_{\mathfrak{m}, \mathfrak{n}}$ the $Y_{\mathfrak{m}, \mathfrak{n}}$-coordinates of $v_{i, j}^{\mathfrak{m}, \mathfrak{m}^{\prime}+\mathfrak{n}}\left(\lambda_{\mathfrak{m}}, \mu\right)$ are the same as

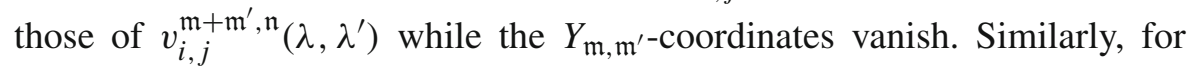
$(i, j) \in X_{\mathfrak{m}, \mathfrak{m}^{\prime}}$, the $Y_{\mathfrak{m}, \mathfrak{m}^{\prime}}$-coordinates of $v_{i, j}^{\mathfrak{m}, \mathfrak{m}^{\prime}+\mathfrak{n}}\left(\lambda_{\mathfrak{m}}, \mu\right)$ are the same as those

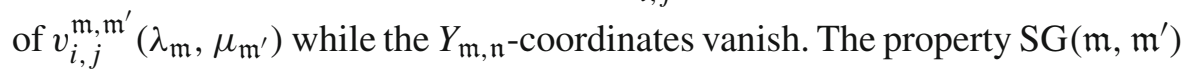
therefore implies $\mathrm{SG}\left(\mathfrak{m}, \mathfrak{m}^{\prime}+\mathfrak{n}\right)$. 
For the third part, write $X_{\mathfrak{m}^{\prime}+\mathfrak{n}}=X_{\mathfrak{m}^{\prime}} \cup X_{\mathfrak{n}} \cup X_{\mathfrak{m}^{\prime}, \mathfrak{n}} \cup X_{\mathfrak{n}, \mathfrak{m}^{\prime}}$ and take $\lambda \in \mathbf{C}_{\mathfrak{m}^{\prime}+\mathfrak{n}}$ whose coordinates with respect to $X_{\mathfrak{m}^{\prime}, \mathfrak{n}} \cup X_{\mathfrak{n}, \mathfrak{m}^{\prime}}$ vanish.

The fourth part is an immediate consequence.

To prove the last part of Proposition 6, recall that $\mathrm{SA}\left(\mathfrak{m}+\mathfrak{m}^{\prime}, \mathfrak{n}\right)$ if and only if the image of the intertwining operator $R_{Z(\mathfrak{n}), Z\left(\mathfrak{m}+\mathfrak{m}^{\prime}\right)}$ is $Z\left(\mathfrak{m}+\mathfrak{m}^{\prime}+\mathfrak{n}\right)$. On the other hand, by assumption $Z\left(\mathfrak{m}+\mathfrak{m}^{\prime}\right)=Z(\mathfrak{m}) \times Z\left(\mathfrak{m}^{\prime}\right)$ and therefore

$$
R_{Z(\mathfrak{n}), Z\left(\mathfrak{m}+\mathfrak{m}^{\prime}\right)}=\left(\mathrm{id} \times R_{Z(\mathfrak{n}), Z\left(\mathfrak{m}^{\prime}\right)}\right) \circ\left(R_{Z(\mathfrak{n}), Z(\mathfrak{m})} \times \mathrm{id}\right) .
$$

The required equivalence follows from the fact that $Z\left(\mathfrak{m}+\mathfrak{m}^{\prime}+\mathfrak{n}\right) \unlhd Z(\mathfrak{n}) \times$ $Z\left(\mathfrak{m}+\mathfrak{m}^{\prime}\right), \operatorname{JH}\left(Z(\mathfrak{n}+\mathfrak{m}) \times Z\left(\mathfrak{m}^{\prime}\right)\right)$ and $\operatorname{JH}\left(Z\left(\mathfrak{n}+\mathfrak{m}^{\prime}\right) \times Z(\mathfrak{m})\right)$ and that the images of $R_{Z(\mathfrak{n}), Z\left(\mathfrak{m}+\mathfrak{m}^{\prime}\right)}, R_{Z(\mathfrak{n}), Z(\mathfrak{m})}$ and $R_{Z(\mathfrak{n}), Z\left(\mathfrak{m}^{\prime}\right)}$ are irreducible.

This finishes the proof of Proposition 6.

The second part of Theorem 1 now follows by induction on $k$.

\subsection{Proof of Proposition 7}

The first part is a special case of (13). We prove the second part. Observe that $X_{\mathfrak{n}}=X_{\mathfrak{m}} \cup X_{\mathfrak{m}^{\prime}} \cup X_{\mathfrak{m}, \mathfrak{m}^{\prime}} \cup X_{\mathfrak{m}^{\prime}, \mathfrak{m}}$ and $Y_{\mathfrak{n}}=Y_{\mathfrak{m}} \cup Y_{\mathfrak{m}^{\prime}} \cup Y_{\mathfrak{m}, \mathfrak{m}^{\prime}} \cup Y_{\mathfrak{m}^{\prime}, \mathfrak{m}}$. Assume that $\lambda \in \mathbf{C}_{\mathfrak{n}}^{l^{\prime}}$. Let $\lambda_{\mathfrak{m}}$ and $\lambda_{\mathfrak{m}^{\prime}}$ be as in Sect. 8.5. Then, for any $(i, j) \in X_{\mathfrak{n}}$

1. The $Y_{\mathfrak{n}, \mathfrak{m}}$-coordinates of $v_{i, j}^{\mathfrak{n}}(\lambda)$ are $v_{i, j}^{\mathfrak{n}, \mathfrak{m}}\left(\lambda, \lambda_{\mathfrak{m}}\right)$ if $(i, j) \in X_{\mathfrak{n}, \mathfrak{m}}$ and 0 otherwise.

2. If $(i, j) \in X_{\mathfrak{n}, \mathfrak{m}^{\prime}}$ then the $Y_{\mathfrak{m}^{\prime}}$-coordinates of $v_{i, j}^{\mathfrak{n}}(\lambda)$ are $v_{i, j}^{\mathfrak{m}^{\prime}}\left(\lambda_{\mathfrak{m}^{\prime}}\right)$ if $(i, j) \in$ $X_{\mathfrak{m}^{\prime}}$ and 0 otherwise.

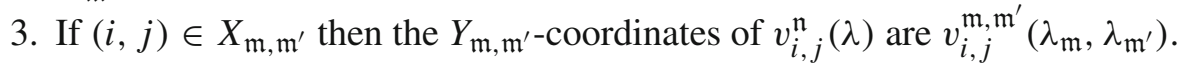

It follows from the assumptions $G L S\left(\mathfrak{m}^{\prime}\right)$ and $\operatorname{SG}\left(\mathfrak{m}, \mathfrak{m}^{\prime}\right)$ that in order to show $G L S(\mathfrak{n})$, it is enough to know that $v_{i, j}^{\mathfrak{n}, \mathfrak{m}}\left(\lambda, \lambda_{\mathfrak{m}}\right),(i, j) \in X_{\mathfrak{n}, \mathfrak{m}}$ are linearly independent for generic $\lambda \in \mathbf{C}_{\mathfrak{m}^{\prime}, \mathfrak{m}}$. By the condition $\operatorname{SG}(\mathfrak{n}, \mathfrak{m}), v_{i, j}^{\mathfrak{n}, \mathfrak{m}}\left(\lambda, \lambda^{\prime}\right)$, $(i, j) \in X_{\mathfrak{n}, \mathfrak{m}}$ are linearly independent for generic $\left(\lambda, \lambda^{\prime}\right) \in \mathbf{C}_{\mathfrak{n}} \times \mathbf{C}_{\mathfrak{m}}$. Since this condition in $\lambda$ is invariant under the action of $G_{\mathfrak{n}}$, it follows from Proposition 3 and the condition $\operatorname{SG}\left(\mathfrak{m}, \mathfrak{m}^{\prime}\right)$ that $v_{i, j}^{\mathfrak{n}, \mathfrak{m}}\left(\lambda, \lambda^{\prime}\right),(i, j) \in X_{\mathfrak{n}, \mathfrak{m}}$ are linearly independent for generic $\left(\lambda, \lambda^{\prime}\right) \in \mathbf{C}_{\mathfrak{n}}^{l^{\prime}} \times \mathbf{C}_{\mathfrak{m}}$. Since this condition in $\lambda^{\prime}$ is invariant under the action of $G_{\mathfrak{m}}$, the condition $G L S(\mathfrak{m})$ (which says that $\mathbf{C}_{\mathfrak{m}}$ admits an open $G_{\mathfrak{m}}$-orbit) guarantees that we can take $\lambda^{\prime}=\lambda_{\mathfrak{m}}$, as required.

\subsection{Proof of Proposition 8}

For this subsection fix $\rho \in \operatorname{Irr}_{c}$.

For any $\pi \in$ Irr there exist an integer $m \geq 0$ and $\pi^{\prime} \in$ Irr such that $\pi \hookrightarrow \rho^{\times m} \times \pi^{\prime}$ and $\rho \ltimes \pi^{\prime}$ where $\rho^{\times m}=\overbrace{\rho \times \cdots \times \rho}^{m}$. Moreover, $m$ 
and $\pi^{\prime}$ are unique. (In fact, $m$ is the largest non-negative integer for which $\pi \hookrightarrow \rho^{\times m} \times \pi^{\prime}$ for some $\pi^{\prime} \in \mathcal{M}$.) We write $\mu^{\rho}(\pi)=m$ and ${ }_{\rho} \pi=\pi^{\prime}$ (the left $\rho$-derivative of $\pi$ ). If $\pi=Z(\mathfrak{m})$ and $\pi^{\prime}=Z\left(\mathfrak{m}^{\prime}\right)$, then we also write $\mu^{\rho}(\mathfrak{m})=m$ and ${ }_{\rho} \mathfrak{m}=\mathfrak{m}^{\prime}$.

If $\pi$ is $\square$-irreducible then so is $\rho \pi$ [20, Corollary 2.13]. Similarly, $G L S(\mathfrak{m}) \Longrightarrow G L S\left({ }_{\rho} \mathfrak{m}\right)[20$, Lemma 4.17].

We now recall some results from [13,23] (see also [19,20]).

Let $\mathfrak{m}=\sum_{i \in I} \Delta_{i} \in \mathbb{N}(\Sigma)$. We say that two subsets $A$ and $B$ of $I$ are equivalent and write $A \sim B$ if $\sum_{i \in A} \Delta_{i}=\sum_{i \in B} \Delta_{i}$. Let

$$
X_{\mathfrak{m}}^{\rho}=\left\{i \in I: \rho \prec \Delta_{i}\right\}=\left\{i \in I: b\left(\Delta_{i}\right)=\vec{\rho}\right\}
$$

and

$$
Y_{\mathfrak{m}}^{\rho}=\left\{i \in I: \overleftarrow{\rho} \prec \Delta_{i}\right\}=\left\{i \in I: b\left(\Delta_{i}\right)=\rho\right\}
$$

A one-to-one relation $R \subset Y_{\mathfrak{m}}^{\rho} \times X_{\mathfrak{m}}^{\rho}$ between $Y_{\mathfrak{m}}^{\rho}$ and $X_{\mathfrak{m}}^{\rho}$ is called a $\rho$-matching (with respect to $\mathfrak{m}$ ) if $\Delta_{i} \prec \Delta_{j}$ for all $(i, j) \in R$. We think of $R$ as a partially defined bijection and we write $R(i)=j$ and $R^{-1}(j)=i$ if $(i, j) \in R$. We write

$A(R)=\left\{i \in Y_{\mathfrak{m}}^{\rho}: R(i)\right.$ not defined $\}, B(R)=\left\{j \in X_{\mathfrak{m}}^{\rho}: R^{-1}(j)\right.$ not defined $\}$

Given two $\rho$-matchings $R_{1}, R_{2}$ we say that $R_{2}$ dominates $R_{1}$ if one of the following conditions holds.

1. $R_{2} \supset R_{1}$.

2. There exist $i, j \in Y_{\mathfrak{m}}^{\rho}$ and $k \in X_{\mathfrak{m}}^{\rho}$ such that $R_{2} \backslash R_{1}=\{(j, k)\}, R_{1} \backslash R_{2}=$ $\{(i, k)\}$ and $\Delta_{i}<\Delta_{j}$.

3. There exist $i \in Y_{\mathfrak{m}}^{\rho}$ and $j, k \in X_{\mathfrak{m}}^{\rho}$ such that $R_{2} \backslash R_{1}=\{(i, k)\}, R_{1} \backslash R_{2}=$ $\{(i, j)\}$ and $\Delta_{k}<\Delta_{j}$.

The transitive closure of domination is a partial order on the set of $\rho$-matchings, which we denote by $\leq$. Clearly, if $R \leq R^{\prime}$ then $\sum_{i \in A\left(R^{\prime}\right)} \Delta_{i} \leq \sum_{i \in A(R)} \Delta_{i}$ in the sense that for every $\Delta \in \Sigma, \#\left\{i \in A\left(R^{\prime}\right): \Delta_{i} \geq \Delta\right\} \leq \#\{i \in A(R)$ : $\Delta_{i} \geq \Delta$ \}. Clearly, a $\rho$-matching $R$ is maximal (with respect to $\leq$ ) if and only if whenever $\Delta_{i} \prec \Delta_{j}$ with $(i, j) \in Y_{\mathfrak{m}}^{\rho} \times X_{\mathfrak{m}}^{\rho}$ (exactly) one of the following possibilities occurs.

1. $R(i)$ and $R^{-1}(j)$ are defined.

2. $R(i)$ is defined, $R^{-1}(j)$ is not defined and $\Delta_{j} \geq \Delta_{R(i)}$.

3. $R(i)$ is not defined, $R^{-1}(j)$ is defined and $\Delta_{i} \leq \Delta_{R^{-1}(j)}$. 
We say that two $\rho$-matchings $R_{1}$ and $R_{2}$ are equivalent if

$$
\sum_{(i, j) \in R_{1}}\left(\Delta_{i}, \Delta_{j}\right)=\sum_{(i, j) \in R_{2}}\left(\Delta_{i}, \Delta_{j}\right)
$$

as elements of $\mathbb{N}(\Sigma \times \Sigma)$.

Maximal $\rho$-matchings are not unique up to equivalence. (For instance, we could take $\mathfrak{m}=\Delta_{1}+\Delta_{2}+\Delta_{3}+\Delta_{4}$ such that $X_{\mathfrak{m}}^{\rho}=\{3,4\}, Y_{\mathfrak{m}}^{\rho}=\{1,2\}$, $\Delta_{1} \neq \Delta_{2}, \Delta_{3} \neq \Delta_{4}$ and $\Delta_{i} \prec \Delta_{j}$ for $i=1,2, j=3$, 4. Then both $\{(1,3),(2,4)\}$ and $\{(1,4),(2,3)\}$ are maximal $\rho$-matchings.) However, up to equivalence, $A(R)$ and $B(R)$ are independent of $R$, if $R$ is maximal. We denote them by

$$
A_{\mathfrak{m}}^{\rho} \text { and } B_{\mathfrak{m}}^{\rho} .
$$

(Technically, they are only defined up to equivalence, but this will not matter in what follows.) Moreover,

1. $\rho \mathfrak{m}=\sum_{i \in I} \Delta_{i}^{\prime}$ where

$$
\Delta_{i}^{\prime}= \begin{cases}\Delta_{i} \backslash\{\rho\} & i \in A_{\mathfrak{m}}^{\rho}, \\ \Delta_{i} & \text { otherwise. }\end{cases}
$$

Thus, $\mu^{\rho}(\mathfrak{m})=\# A_{\mathfrak{m}}^{\rho}$ and in particular, $\rho \lessdot Z(\mathfrak{m})$ if and only if $A_{\mathfrak{m}}^{\rho}=\emptyset$. 2. If $B_{\mathfrak{m}}^{\rho}=\emptyset$, then $\operatorname{soc}(\rho \times Z(\mathfrak{m}))=Z(\mathfrak{m}+\{\rho\})$. Otherwise, fix an index $i_{0} \in B_{\mathfrak{m}}^{\rho}$ for which $\Delta_{i_{0}}$ is maximal. Then $\operatorname{soc}(\rho \times Z(\mathfrak{m}))=Z\left(\mathfrak{m}^{\prime}\right)$ where $\mathfrak{m}^{\prime}=\sum_{i \in I} \Delta_{i}^{\prime}$ with $\Delta_{i}^{\prime}= \begin{cases}\{\rho\} \cup \Delta_{i} & \text { if } i=i_{0} \\ \Delta_{i} & \text { otherwise. }\end{cases}$

A maximal $\rho$-matching $R$ between $Y_{\mathfrak{m}}^{\rho}$ and $X_{\mathfrak{m}}^{\rho}$ is called best $\rho$-matching if $\nexists(i, j),\left(i^{\prime}, j^{\prime}\right) \in R$ such that $\Delta_{i}<\Delta_{i^{\prime}} \prec \Delta_{j}<\Delta_{j^{\prime}}$. A best $\rho$-matching exists and is unique up to equivalence. Moreover, a maximal $\rho$-matching $R$ is best if and only if the product of the representations $Z\left(\Delta_{i}\right), i \in A_{\mathfrak{m}}^{\rho} \cup$ $B_{\mathfrak{m}}^{\rho}$ and $Z\left(\Delta_{i}+\Delta_{j}\right),(i, j) \in R$ is irreducible, in which case it is equal to $Z\left(\sum_{i \in I: b\left(\Delta_{i}\right) \in\{\rho, \vec{\rho}\}} \Delta_{i}\right)$.

In particular, if $b\left(\Delta_{i}\right) \in\{\rho, \vec{\rho}\}$ for all $i$, then it follows from Theorem 1 that

$\mathfrak{m}$ is good. Moreover, if $R$ is a best $\rho$-matching, then for any $\mathfrak{m}^{\prime}$,

$$
\begin{aligned}
& \mathrm{SA}\left(\mathfrak{m}, \mathfrak{m}^{\prime}\right) \Longleftrightarrow \mathrm{SA}\left(\Delta_{i}, \mathfrak{m}^{\prime}\right) \forall i \in A_{\mathfrak{m}}^{\rho} \cup B_{\mathfrak{m}}^{\rho} \text { and } \mathrm{SA}\left(\Delta_{i}+\Delta_{j}, \mathfrak{m}^{\prime}\right)(31) \\
& \forall(i, j) \in R \text {. }
\end{aligned}
$$

It is useful to introduce another notion which is weaker than maximality. 
Definition 7 We say that a $\rho$-matching $R$ for $\mathfrak{m}$ is saturated if $X_{\mathfrak{m}} \cap(A(R) \times$ $B(R))=\emptyset$ and for every $(i, j) \in R$ and $i^{\prime} \in A(R)$ such that $\left(i^{\prime}, j\right) \in X_{\mathfrak{m}}$ we have $\Delta_{i^{\prime}} \leq \Delta_{i}$.

Lemma 13 Let $R$ be a saturated $\rho$-matching $R$ for $\mathfrak{m}$. Then, for any $R^{\prime} \geq R$, $R^{\prime}$ is saturated and $A(R)=A\left(R^{\prime}\right)$. Hence, $A(R) \sim A_{\mathfrak{m}}^{\rho}$.

Proof It is enough to show it when $R^{\prime}$ dominates $R$. By the saturation of $R$ it is clear that $R^{\prime}$ is obtained from $R$ by replacing a certain pair $(i, j) \in R$ by $\left(i, j^{\prime}\right) \in X_{\mathfrak{m}}$ where $j^{\prime} \in B(R)$ and $\Delta_{j^{\prime}}<\Delta_{j}$. Thus, $A(R)=A\left(R^{\prime}\right)$ and $B\left(R^{\prime}\right)=B(R) \cup\{j\} \backslash\left\{j^{\prime}\right\}$. Let us show that $R^{\prime}$ is saturated. First, if $\left(i^{\prime}, j\right) \in X_{\mathfrak{m}}$ with $i^{\prime} \in A(R)$ then $\Delta_{i^{\prime}} \leq \Delta_{i}$ by saturation and hence $\left(i^{\prime}, j^{\prime}\right) \in X_{\mathfrak{m}}$ (since $\left.\left(i, j^{\prime}\right) \in X_{\mathfrak{m}}\right)$. Thus, $X_{\mathfrak{m}} \cap\left(A\left(R^{\prime}\right) \times B\left(R^{\prime}\right)\right)=\emptyset$. On the other hand, we cannot have $i^{\prime} \in A(R)$ with $\left(i^{\prime}, j^{\prime}\right) \in X_{\mathfrak{m}}$ since $R$ is saturated. Therefore, the second condition for saturation for $R^{\prime}$ follows from the saturation of $R$.

For any $\mathfrak{m}^{\prime} \in \mathbb{N}(\Sigma)$ let

$$
\tilde{X}_{\mathfrak{m}, \mathfrak{m}^{\prime}}^{\rho}=X_{\mathfrak{m}, \mathfrak{m}^{\prime}} \cap\left(A_{\mathfrak{m}}^{\rho} \times X_{\mathfrak{m}^{\prime}}^{\rho}\right), \quad \tilde{Y}_{\mathfrak{m}, \mathfrak{m}^{\prime}}^{\rho}=Y_{\mathfrak{m}, \mathfrak{m}^{\prime}} \cap\left(A_{\mathfrak{m}}^{\rho} \times Y_{\mathfrak{m}^{\prime}}^{\rho}\right) .
$$

(See (29a), (29b) and (30).) As usual, we write $\tilde{X}_{\mathfrak{m}}^{\rho}=\tilde{X}_{\mathfrak{m}, \mathfrak{m}}^{\rho}$.

Lemma 14 Suppose that $\rho \in Z\left(\mathfrak{m}^{\prime}\right)$. Then, $\# \tilde{X}_{\mathfrak{m}, \mathfrak{m}^{\prime}}^{\rho} \geq \# \tilde{Y}_{\mathfrak{m}, \mathfrak{m}^{\prime}}^{\rho}$. Moreover, equality holds if and only if

$$
\operatorname{soc}\left(\rho^{\times \mu^{\rho}(\mathfrak{m})} \times Z\left({ }_{\rho} \mathfrak{m}+\mathfrak{m}^{\prime}\right)\right)=Z\left(\mathfrak{m}+\mathfrak{m}^{\prime}\right) .
$$

Proof Let $R$ and $R^{\prime}$ be maximal $\rho$-matchings with respect to $\mathfrak{m}$ and $\mathfrak{m}^{\prime}$ respectively. By assumption, $A_{\mathfrak{m}^{\prime}}^{\rho}=\emptyset$. Hence, the function $f: \tilde{Y}_{\mathfrak{m}, \mathfrak{m}^{\prime}}^{\rho} \rightarrow \tilde{X}_{\mathfrak{m}, \mathfrak{m}^{\prime}}^{\rho}$ given by $f\left(i, j^{\prime}\right)=\left(i, R^{\prime}\left(j^{\prime}\right)\right)$ is well-defined and injective.

Since $R \cup R^{\prime}$ is a $\rho$-matching with respect to $\mathfrak{m}+\mathfrak{m}^{\prime}$, we have $\mu^{\rho}\left(\mathfrak{m}+\mathfrak{m}^{\prime}\right)=$ $\# A_{\mathfrak{m}+\mathfrak{m}^{\prime}}^{\rho} \leq \# A_{\mathfrak{m}}^{\rho}=\mu^{\rho}(\mathfrak{m})$. By the above descriptions of ${ }_{\rho} \mathfrak{m}$ and $\operatorname{soc}(\rho \times *)$, the condition (33) means that $A_{\mathfrak{m}+\mathfrak{m}^{\prime}}^{\rho} \sim A_{\mathfrak{m}}^{\rho}$. We show that this is equivalent to the surjectivity of $f$. Suppose that $f$ is not onto and let $\left(i, j^{\prime}\right) \in \tilde{X}_{\mathfrak{m}, \mathfrak{m}^{\prime}}^{\rho}$ be outside the image of $f$. Then, either $j^{\prime} \in B_{\mathfrak{m}^{\prime}}^{\rho}$, in which case $R \cup R^{\prime} \cup\left\{\left(i, j^{\prime}\right)\right\}$ dominates $R \cup R^{\prime}$, or $\Delta_{i^{\prime}}<\Delta_{i}$ where $i^{\prime}=R^{\prime-1}\left(j^{\prime}\right)$, in which case $R \cup R^{\prime} \cup$ $\left\{\left(i, j^{\prime}\right)\right\} \backslash\left\{\left(i^{\prime}, j^{\prime}\right)\right\}$ dominates $R \cup R^{\prime}$. In both cases $\sum_{i \in A_{\mathfrak{m}+\mathfrak{m}^{\prime}}^{\rho}} \Delta_{i}<\sum_{i \in A_{\mathfrak{m}}^{\rho}} \Delta_{i}$. Conversely, suppose that $f$ is onto. Then, $R \cup R^{\prime}$ is saturated (with respect to $\left.\mathfrak{m}+\mathfrak{m}^{\prime}\right)$. It follows from Lemma 13 that $A_{\mathfrak{m}+\mathfrak{m}^{\prime}}^{\rho} \sim A_{\mathfrak{m}}^{\rho}$ as required.

The following result follows from Lemma 5.

Lemma 15 Suppose that $\pi \in \operatorname{Irr}$ with $\rho \Uparrow \pi$ and let $m \geq 1$. Then, $\rho^{\times m} \times \pi$ is $S I, \mu^{\rho}\left(\operatorname{soc}\left(\rho^{\times m} \times \pi\right)\right)=m$ and if $\pi^{\prime}$ is any subquotient of $\rho^{\times m} \times \pi$ other than $\operatorname{soc}\left(\rho^{\times m} \times \pi\right)$ then $\mu^{\rho}\left(\pi^{\prime}\right)<m$. 
Corollary 9 Suppose that $\pi^{\prime} \in \operatorname{Irr}$ with $\rho \gtrless \pi^{\prime}$. Then, for any $\pi \in \operatorname{Irr}$ we have

$$
\operatorname{soc}\left(\pi \times \pi^{\prime}\right)=\operatorname{soc}\left(\rho^{\times m} \times \rho_{\rho} \times \pi^{\prime}\right)=\operatorname{soc}\left(\rho^{\times m} \times \operatorname{soc}\left(\rho \pi \times \pi^{\prime}\right)\right) .
$$

Proof Since $\rho \gtrless{ }_{\rho} \pi$ and $\rho \ll \pi^{\prime}$, we have $\rho \ll{ }_{\rho} \pi \times \pi^{\prime}$. The second equality of (34) follows from Lemma 6 . To prove the first equality suppose on the contrary that $\sigma$ is a subrepresentation of $\rho^{\times m} \times \rho_{\rho} \times \pi^{\prime}$ which is not a subrepresentation of $\pi \times \pi^{\prime}$. Then, $\sigma \hookrightarrow \tau \times \pi^{\prime}$ for some irreducible subquotient $\tau$ of $\rho^{\times m} \times{ }_{\rho} \pi$ other than $\pi$. But then, $\mu^{\rho}(\sigma)=\mu^{\rho}(\tau)<m$. On the other hand, we already know by the second equality of (34) that $\sigma \hookrightarrow$ $\rho^{\times m} \times \sigma^{\prime}$ for some $\sigma^{\prime}$. We get a contradiction.

Lemma 16 Let $R$ be a $\rho$-matching for $\mathfrak{m}$. Then, any non-empty open $G_{\mathfrak{m}}$ invariant subset $S$ of $\mathbf{C}_{\mathfrak{m}}$ contains an element $\lambda$ such that $\lambda_{i, R(j)}=\delta_{i, j}$ for all $(i, j) \in Y_{\mathfrak{m}} \cap\left(Y_{\mathfrak{m}}^{\rho} \times Y_{\mathfrak{m}}^{\rho}\right)$ such that $R(j)$ is defined.

Proof Let $\left\{j_{1}, \ldots, j_{r}\right\}$ be the domain of $R$ with $\Delta_{j_{1}} \geq \cdots \geq \Delta_{j_{r}}$. We show by induction on $l$ that we can find $\lambda \in S$ such that $\lambda_{i, R\left(j_{m}\right)}=\delta_{i, j_{m}}$ for all $m=1, \ldots, l$ and all $i \in Y_{\mathfrak{m}}^{\rho}$ such that $\left(i, j_{m}\right) \in Y_{\mathfrak{m}}$. The case $l=0$ is trivial. For the induction step, let $0<l \leq r$ and assume that the statement holds for $l-1$. By openness, we may assume in addition that $\lambda$ satisfies $\lambda_{j_{l}, R\left(j_{l}\right)} \neq 0$. Define $g \in G_{\mathfrak{m}}$ by

$$
g_{i, j}=\left\{\begin{array}{ll}
\lambda_{i, R\left(j_{l}\right)} & j=j_{l}, \\
\delta_{i, j} & \text { otherwise, }
\end{array}(i, j) \in Y_{\mathfrak{m}} .\right.
$$

Note that $g$ is invertible because $\lambda_{j_{l}, R\left(j_{l}\right)} \neq 0$.

Let $\tilde{\lambda}=g^{-1} \lambda g$. Then, $g x_{\rho}, R\left(j_{l}\right)=x_{\rho, R\left(j_{l}\right)}$ and

$$
\lambda x_{\rho, R\left(j_{l}\right)}=\sum_{i} \lambda_{i, R\left(j_{l}\right)} x_{\rho, i}=g x_{\rho, j_{l}}+\sum_{i:\left(i, j_{l}\right) \notin Y_{\mathfrak{m}}} \lambda_{i, R\left(j_{l}\right)} x_{\rho, i} .
$$

Thus,

$$
\tilde{\lambda}_{\vec{\rho}, R\left(j_{l}\right)}=x_{\rho, j_{l}}+\sum_{i:\left(i, j_{l}\right) \notin Y_{\mathfrak{m}}} \lambda_{i, R\left(j_{l}\right)} x_{\rho, i} .
$$

It follows that $\tilde{\lambda}_{i, R\left(j_{l}\right)}=\delta_{i, j_{l}}$ for all $i \in Y_{\mathfrak{m}}^{\rho}$ such that $\left(i, j_{l}\right) \in Y_{\mathfrak{m}}$. Now let $m<l$. Then, $g x_{\vec{\rho}, R\left(j_{m}\right)}=x_{\vec{\rho}, R\left(j_{m}\right)}$ and by induction hypothesis

$$
\lambda x_{\rho, R\left(j_{m}\right)}=x_{\rho, j_{m}}+\xi
$$


where the coordinate of $\xi$ with respect to $x_{\rho, i}$ is zero unless $i \notin Y_{\mathfrak{m}}^{\rho}$ or $\Delta_{i}>$ $\Delta_{j_{m}}$. Since $\Delta_{j_{l}} \leq \Delta_{j_{m}}$ it follows that

$$
\tilde{\lambda}_{\vec{\rho}, R\left(j_{m}\right)}=\lambda x_{\vec{\rho}, R\left(j_{m}\right)}=x_{\rho, j_{m}}+\xi .
$$

This completes the induction hypothesis.

By passing to the contragredient we get

Lemma 17 Let $R$ be a $\rho$-matching for $\mathfrak{m}$. Then, any non-empty open $G_{\mathfrak{m}}$ invariant subset $S$ of $\mathbf{C}_{\mathfrak{m}}$ contains an element $\lambda$ such that $\lambda_{R^{-1}(i), j}=\delta_{i, j}$ for all $(i, j) \in Y_{\mathfrak{m}} \cap\left(X_{\mathfrak{m}}^{\rho} \times X_{\mathfrak{m}}^{\rho}\right)$ such that $R^{-1}(i)$ is defined.

Finally, we can prove Proposition 8.

By Lemma 14 and Corollary 9, if $\# \tilde{X}_{\mathfrak{m}, \mathfrak{m}^{\prime}}^{\rho}=\# \tilde{Y}_{\mathfrak{m}, \mathfrak{m}^{\prime}}^{\rho}$, then the conditions $\operatorname{SA}\left({ }_{\rho} \mathfrak{m}, \mathfrak{m}^{\prime}\right)$ and $\mathrm{SA}\left(\mathfrak{m}, \mathfrak{m}^{\prime}\right)$ are equivalent. We show that $\mathrm{SA}\left(\mathfrak{m}, \mathfrak{m}^{\prime}\right)$ implies $\# \tilde{X}_{\mathfrak{m}, \mathfrak{m}^{\prime}}^{\rho}=\# \tilde{Y}_{\mathfrak{m}, \mathfrak{m}^{\prime}}^{\rho}$. By Corollary 4, we may assume that $b\left(\Delta_{i}\right) \in\{\rho, \vec{\rho}\}$ for all $i \in I \cup I^{\prime}$. If $\mathrm{SA}\left(\mathfrak{m}, \mathfrak{m}^{\prime}\right)$, then it follows from (31) that $\mathrm{SA}\left(\Delta_{i}, \mathfrak{m}^{\prime}\right)$ for every $i \in A_{\mathfrak{m}}^{\rho}$. Taking a best matching $R^{\prime}$ for $\mathfrak{m}^{\prime}$, this means that $\operatorname{SA}\left(\Delta_{i}, \Delta_{j^{\prime}}\right)$ for every $j^{\prime} \in B_{\mathfrak{m}^{\prime}}^{\rho}$ and $\operatorname{SA}\left(\Delta_{i}, \Delta_{i^{\prime}}+\Delta_{j^{\prime}}\right)$ for every $\left(i^{\prime}, j^{\prime}\right) \in R^{\prime}$. This exactly means that the function $f$ defined in the proof of Lemma 14 is surjective, i.e. that $\# \tilde{X}_{\mathfrak{m}, \mathfrak{m}^{\prime}}^{\rho}=\# \tilde{Y}_{\mathfrak{m}, \mathfrak{m}^{\prime}}^{\rho}$.

For the second part, note that $X_{\rho \mathfrak{m}, \mathfrak{m}^{\prime}} \subseteq X_{\mathfrak{m}, \mathfrak{m}^{\prime}}, Y_{\rho \mathfrak{m}, \mathfrak{m}^{\prime}} \subseteq Y_{\mathfrak{m}, \mathfrak{m}^{\prime}}$ and

$$
\tilde{X}_{\mathfrak{m}, \mathfrak{m}^{\prime}}^{\rho}=X_{\mathfrak{m}, \mathfrak{m}^{\prime}} \backslash X_{\rho \mathfrak{m}, \mathfrak{m}^{\prime}}, \quad \tilde{Y}_{\mathfrak{m}, \mathfrak{m}^{\prime}}^{\rho}=Y_{\mathfrak{m}, \mathfrak{m}^{\prime}} \backslash Y_{\rho \mathfrak{m}, \mathfrak{m}^{\prime}} .
$$

Also, we have

$$
X_{\mathfrak{m}} \backslash X_{\rho \mathfrak{m}}=\tilde{X}_{\mathfrak{m}, \mathfrak{m}}^{\rho} \cup \hat{X}_{\mathfrak{m}} \text { where } \hat{X}_{\mathfrak{m}}=\left\{i \in I: e\left(\Delta_{i}\right)=\overleftarrow{\rho}\right\} \times A_{\mathfrak{m}}^{\rho}
$$

and

$$
X_{\rho \mathfrak{m}} \backslash X_{\mathfrak{m}}=X_{\rho \mathfrak{m}} \cap\left(Y_{\mathfrak{m}}^{\rho} \times A_{\mathfrak{m}}^{\rho}\right) .
$$

Suppose that $\operatorname{SG}\left(\mathfrak{m}, \mathfrak{m}^{\prime}\right)$. Let $\left(\lambda, \lambda^{\prime}\right) \in \mathbf{C}_{\mathfrak{m}} \times \mathbf{C}_{\mathfrak{m}^{\prime}}$ be such that $v_{i, j}^{\mathfrak{m}, \mathfrak{m}^{\prime}}\left(\lambda, \lambda^{\prime}\right)$,

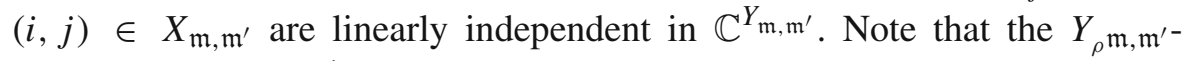
coordinates of $v_{i, j}^{\mathfrak{m}, \mathfrak{m}^{\prime}}\left(\lambda, \lambda^{\prime}\right),(i, j) \in X_{\mathfrak{m}, \mathfrak{m}^{\prime}}$ are independent of the $\hat{X}_{\mathfrak{m}}$ coordinates of $\lambda$. By Lemma 16 (for $R$ maximal) we may assume that $\lambda_{i, j}=0$ for all $(i, j) \in \tilde{X}_{\mathfrak{m}}^{\rho}$. This guarantees that

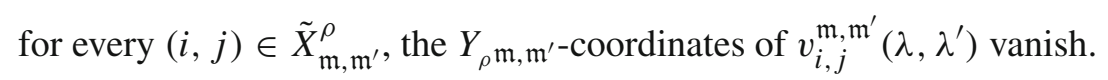


Hence, $\# \tilde{X}_{\mathfrak{m}, \mathfrak{m}^{\prime}}^{\rho} \leq \# \tilde{Y}_{\mathfrak{m}, \mathfrak{m}^{\prime}}^{\rho}$. Thus, Lemma $14 \tilde{X}_{\mathfrak{m}, \mathfrak{m}^{\prime}}^{\rho}=\# \tilde{Y}_{\mathfrak{m}, \mathfrak{m}^{\prime}}^{\rho}$. The vanishing of $\lambda_{i, j}=0$ for $(i, j) \in \tilde{X}_{\mathfrak{m}}^{\rho}$ also guarantees that

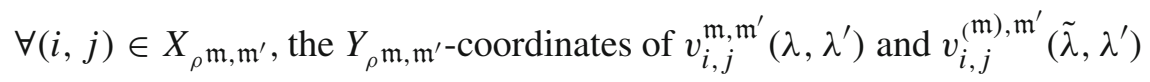

where $\tilde{\lambda} \in \mathbf{C}_{\rho}$ m is given by

$$
\tilde{\lambda}_{i, j}= \begin{cases}\lambda_{i, j} & (i, j) \in X_{(\mathfrak{m})} \cap X_{\mathfrak{m}}, \\ 0 & (i, j) \in X_{(\mathfrak{m})} \backslash X_{\mathfrak{m}} .\end{cases}
$$

Thus, $\operatorname{SG}\left(\mathfrak{m}, \mathfrak{m}^{\prime}\right) \Longrightarrow \operatorname{SG}\left({ }_{\rho} \mathfrak{m}, \mathfrak{m}^{\prime}\right)$.

Conversely, suppose that $\operatorname{SG}\left({ }_{\rho} \mathfrak{m}, \mathfrak{m}^{\prime}\right)$ is satisfied and $\# \tilde{X}_{\mathfrak{m}, \mathfrak{m}^{\prime}}^{\rho}=\# \tilde{Y}_{\mathfrak{m}, \mathfrak{m}^{\prime}}^{\rho}$. Let $\left(\tilde{\lambda}, \lambda^{\prime}\right) \in \mathbf{C}_{\rho \mathfrak{m}} \times \mathbf{C}_{\mathfrak{m}^{\prime}}$ be such that $v_{i, j}^{\rho^{\mathfrak{m}}, \mathfrak{m}^{\prime}}\left(\tilde{\lambda}, \lambda^{\prime}\right),(i, j) \in X_{\rho \mathfrak{m}, \mathfrak{m}^{\prime}}$ are linearly independent in $\mathbb{C}^{Y}{ }_{\rho \mathfrak{m}, \mathfrak{m}^{\prime}}$. By Lemma 17 (applied to ${ }_{\rho} \mathfrak{m}$ and a maximal $R$ ) we may assume that $\tilde{\lambda}_{i, j}=0$ for $(i, j) \in X_{\rho \mathfrak{m}} \backslash X_{\mathfrak{m}}$. Define $\lambda \in \mathbf{C}_{\mathfrak{m}}$ by

$$
\lambda_{i, j}= \begin{cases}\tilde{\lambda}_{i, j} & (i, j) \in X_{\mathfrak{m}} \cap X_{\rho \mathfrak{m}}, \\ 0 & (i, j) \in X_{\mathfrak{m}} \backslash X_{\rho \mathfrak{m}} .\end{cases}
$$

Then, (35) and (36) are satisfied. Consider the (square) matrix $M^{\prime}\left(\lambda^{\prime}\right)$ of size $\# \tilde{X}_{\mathfrak{m}, \mathfrak{m}^{\prime}}^{\rho} \times \# \tilde{Y}_{\mathfrak{m}, \mathfrak{m}^{\prime}}^{\rho}$ formed by the $\tilde{Y}_{\mathfrak{m}, \mathfrak{m}^{\prime}}^{\rho}$-coordinates of $v_{i, j}^{\mathfrak{m}, \mathfrak{m}^{\prime}}\left(\lambda, \lambda^{\prime}\right)$, $(i, j) \in \tilde{X}_{\mathfrak{m}, \mathfrak{m}^{\prime}}^{\rho}$ It is independent of $\lambda$ and depends only on the $X_{\mathfrak{m}^{\prime}} \cap\left(Y_{\mathfrak{m}^{\prime}}^{\rho} \times\right.$ $X_{\mathfrak{m}^{\prime}}^{\rho}$ )-coordinates of $\lambda^{\prime}$ (in fact, it does not depend on the $X_{\mathfrak{m}^{\prime}} \cap\left(Y_{\mathfrak{m}^{\prime}}^{\rho} \times B_{\mathfrak{m}^{\prime}}^{\rho}\right)$ coordinates of $\left.\lambda^{\prime}\right)$. To conclude $\mathrm{SG}\left(\mathfrak{m}, \mathfrak{m}^{\prime}\right)$ it remains to show that $M^{\prime}(\lambda)$ is non-singular for generic $\lambda^{\prime} \in \mathbf{C}_{\mathfrak{m}^{\prime}}$, or equivalently for $\lambda^{\prime} \in \mathbf{C}_{\mathfrak{m}^{\prime}}$ of our choice. As in the proof of Lemma 14 , let $R^{\prime}$ be a maximal $\rho$-matching with respect to $\mathfrak{m}^{\prime}$ and let $f: \tilde{Y}_{\mathfrak{m}, \mathfrak{m}^{\prime}}^{\rho} \rightarrow \tilde{X}_{\mathfrak{m}, \mathfrak{m}^{\prime}}^{\rho}$ be the injective function given by $f\left(i, j^{\prime}\right)=\left(i, R^{\prime}\left(j^{\prime}\right)\right)$. Since $\# \tilde{X}_{\mathfrak{m}, \mathfrak{m}^{\prime}}^{\rho}=\# \tilde{Y}_{\mathfrak{m}, \mathfrak{m}^{\prime}}^{\rho}, f$ is onto. Taking $\lambda^{\prime} \in \mathbf{C}_{\mathfrak{m}^{\prime}}$ such that $\lambda_{i^{\prime}, j^{\prime}}^{\prime}=\delta_{j^{\prime}, R^{\prime}\left(i^{\prime}\right)}$ for all $\left(i^{\prime}, j^{\prime}\right) \in X_{\mathfrak{m}^{\prime}} \cap\left(Y_{\mathfrak{m}^{\prime}}^{\rho} \times X_{\mathfrak{m}^{\prime}}^{\rho}\right), M^{\prime}\left(\lambda^{\prime}\right)$ becomes the permutation matrix representing $f$. Our claim follows.

Acknowledgements Open access funding provided by University of Vienna. We would like to thank David Kazhdan for useful discussions. The first-named author would like to thank the University of Vienna and the Institute for Mathematical Sciences, National University of Singapore for their hospitality and support. The second-named author would like to thank the University of Seville for its hospitality and support. Both authors would like to thank the University Institute of Mathematics Research of the University of Seville for its hospitality and support. Last, but not least, we thank the referee for making useful suggestions. 
Open Access This article is licensed under a Creative Commons Attribution 4.0 International License, which permits use, sharing, adaptation, distribution and reproduction in any medium or format, as long as you give appropriate credit to the original author(s) and the source, provide a link to the Creative Commons licence, and indicate if changes were made. The images or other third party material in this article are included in the article's Creative Commons licence, unless indicated otherwise in a credit line to the material. If material is not included in the article's Creative Commons licence and your intended use is not permitted by statutory regulation or exceeds the permitted use, you will need to obtain permission directly from the copyright holder. To view a copy of this licence, visit http://creativecommons.org/licenses/by/4.0/.

\section{References}

1. Arakawa, T., Suzuki, T.: Duality between $\mathfrak{s l}_{n}(C)$ and the degenerate affine Hecke algebra. J. Algebra 209(1), 288-304 (1998)

2. Aubert, A.-M.: Dualité dans le groupe de Grothendieck de la catégorie des représentations lisses de longueur finie d'un groupe réductif $p$-adique. Trans. Am. Math. Soc. 347(6), 2179-2189 (1995)

3. Aubert, A.-M.: Erratum: "Duality in the Grothendieck group of the category of finite-length smooth representations of a $p$-adic reductive group" [Trans. Amer. Math. Soc. 347(6), 1995, pp. 2179-2189; MR1285969 (95i:22025)]: Trans. Amer. Math. Soc. 348(11), 4687-4690 (1996)

4. Bernstein, I.N., Zelevinsky, A.V.: Representations of the group $G L(n, F)$, where $F$ is a local non-Archimedean field. Uspehi Mat. Nauk 31(3(189)), 5-70 (1976)

5. Bernstein, I.N., Zelevinsky, A.V.: Induced representations of reductive p-adic groups. I. Ann. Sci. École Norm. Sup. (4) 10(4), 441-472 (1977)

6. Bernstein, J., Bezrukavnikov, R., Kazhdan, D.: Deligne-Lusztig duality and wonderful compactification. Selecta Math. (N.S.) 24(1), 7-20 (2018)

7. Bernstein, J.N.: $P$-invariant distributions on $\mathrm{GL}(N)$ and the classification of unitary representations of GL $(N)$ (non-Archimedean case). Lie group representations, II (College Park, Md., 1982/1983), pp. 50-102 (1984)

8. Deligne, P.: Catégories tannakiennes. The Grothendieck Festschrift, Vol. II, pp. 111-195 (1990)

9. Geiss, C., Leclerc, B., Schröer, J.: Semicanonical bases and preprojective algebras. Ann. Sci. École Norm. Sup. (4) 38(2), 193-253 (2005)

10. Geiß, C., Leclerc, B., Schröer, J.: Kac-Moody groups and cluster algebras. Adv. Math. 228(1), 329-433 (2011)

11. Hernandez, D., Leclerc, B.: Cluster algebras and quantum affine algebras. Duke Math. J. 154(2), 265-341 (2010)

12. Hernandez, D., Leclerc, B.: Monoidal categorifications of cluster algebras of type $A$ and $D$. In: Symmetries, Integrable Systems and Representations, pp. 175-193. Springer, London (2013)

13. Jantzen, C.: Jacquet modules of $p$-adic general linear groups. Represent. Theory 11, 45-83 (2007). (electronic)

14. Kang, S.-J., Kashiwara, M., Kim, M., Oh, S.: Simplicity of heads and socles of tensor products. Compos. Math. 151(2), 377-396 (2015)

15. Kang, S.-J., Kashiwara, M., Kim, M., Oh, S.: Monoidal categorification of cluster algebras. J. Am. Math. Soc. 31(2), 349-426 (2018)

16. Knight, H., Zelevinsky, A.: Representations of quivers of type $A$ and the multisegment duality. Adv. Math. 117(2), 273-293 (1996)

17. Lapid, E.: Explicit decomposition of certain induced representations of the general linear group (2019). arXiv:1911.04270 
18. Lapid, E., Mínguez, A.: On a determinantal formula of Tadić. Am. J. Math. 136(1), 111-142 (2014)

19. Lapid, E., Mínguez, A.: On parabolic induction on inner forms of the general linear group over a non-archimedean local field. Selecta Math. (N.S.) 22(4), 2347-2400 (2016)

20. Lapid, E., Mínguez, A.: Geometric conditions for $\square$-irreducibility of certain representations of the general linear group over a non-archimedean local field. Adv. Math. 339, 113-190 (2018)

21. Lapid, E.M., Mao, Z.: Local Rankin-Selberg integrals for Speh representations. Compos. Math. 156(5), 908-945 (2020)

22. Leclerc, B.: Imaginary vectors in the dual canonical basis of $U_{q}(\mathfrak{n})$. Transform. Groups 8(1), 95-104 (2003)

23. Mínguez, A.: Sur l'irréductibilité d'une induite parabolique. J. Reine Angew. Math. 629, 107-131 (2009)

24. Mœglin, C., Waldspurger, J.-L.: Sur l'involution de Zelevinski. J. Reine Angew. Math. 372, 136-177 (1986)

25. Pjaseckiū, V.S.: Linear Lie groups that act with a finite number of orbits. Funkcional. Anal. i Priložen. 9(4), 85-86 (1975)

26. Schneider, P., Stuhler, U.: Representation theory and sheaves on the Bruhat-Tits building. Inst. Hautes Études Sci. Publ. Math. 85, 97-191 (1997)

27. Tadić, M.: Classification of unitary representations in irreducible representations of general linear group (non-Archimedean case). Ann. Sci. École Norm. Sup. (4) 19(3), 335-382 (1986)

28. Waldspurger, J.-L.: La formule de Plancherel pour les groupes $p$-adiques (d'après HarishChandra). J. Inst. Math. Jussieu 2(2), 235-333 (2003)

29. Zelevinsky, A.V.: The $p$-adic analogue of the Kazhdan-Lusztig conjecture. Funktsional. Anal. i Prilozhen. 15(2), 9-21 (1981). 96

30. Zelevinsky, A.V.: Two remarks on graded nilpotent classes. Uspekhi Mat. Nauk 40(1(241)), 199-200 (1985)

31. Zelevinsky, A.V.: Induced representations of reductive p-adic groups. II. On irreducible representations of GL(n). Ann. Sci. École Norm. Sup. (4) 13(2), 165-210 (1980)

Publisher's Note Springer Nature remains neutral with regard to jurisdictional claims in published maps and institutional affiliations. 